University of Louisville

ThinkIR: The University of Louisville's Institutional Repository

Electronic Theses and Dissertations

4-2014

\title{
Synthesis of cadmium selenide quantum dots for fabrication of hybrid light emitting diodes.
}

Michael McCreary 1991-
University of Louisville

Follow this and additional works at: https://ir.library.louisville.edu/etd

\section{Recommended Citation}

McCreary, Michael 1991-, "Synthesis of cadmium selenide quantum dots for fabrication of hybrid light emitting diodes." (2014). Electronic Theses and Dissertations. Paper 941.

https://doi.org/10.18297/etd/941

This Master's Thesis is brought to you for free and open access by ThinkIR: The University of Louisville's Institutional Repository. It has been accepted for inclusion in Electronic Theses and Dissertations by an authorized administrator of ThinkIR: The University of Louisville's Institutional Repository. This title appears here courtesy of the author, who has retained all other copyrights. For more information, please contact thinkir@louisville.edu. 


\title{
SYNTHESIS OF CADMIUM SELENIDE QUANTUM DOTS FOR FABRICATION OF HYBRID LIGHT EMITTING DIODES
}

\author{
By \\ Michael Zachary McCreary \\ B.S. Ch.E., University of Louisville, May 2013
}

\begin{abstract}
A Thesis
Submitted to the Faculty of the

University of Louisville

J. B. Speed School of Engineering

as Partial Fulfillment of the Requirements

for the Professional Degree
\end{abstract}

\section{MASTER OF ENGINEERING}

Department of Chemical Engineering

April 2014 


\section{SYNTHESIS OF CADMIUM SELENIDE QUANTUM DOTS FOR FABRICATION OF HYBRID LIGHT EMITTING DIODES}

Submitted By:

Michael Zachary McCreary

A Thesis Approved on

(Date)

By the Following Reading and Examination Committee:

Delaina A. Amos, Thesis Director

Mahendra K. Sunkara

Robert W. Cohn

Gerold A. Willing 


\section{ACKNOWLEDGEMENTS}

First of all, I would like to thank my parents for supporting me in all aspects over the last four years and providing me with this wonderful and rewarding experience. It is only because of their love that I have become who I am today but it goes without saying that there are countless other people that have guided me along the way.

For providing me with an opportunity to test myself in the world of research, I have to thank Dr. Delaina Amos. She gave me the opportunity to participate in undergraduate research which led into the research project presented here.

Menaka Jha has been a tremendous help in the completion of this work and has offered expertise in quantum dot synthesis. None of this would have been possible without her help.

I would like to thank Dr. Sunkara, Dr. Cohn, Dr. Amos, and Dr. Willing for serving on my reading and examination committee and their suggestions for improvements. 
I have to thank Dr. Sunkara for the use of Conn Center for Renewable Energy Research facilities, including the state-of-the-art materials characterization lab. 


\begin{abstract}
The synthesis of CdSe quantum dots was carried out using a variety of methods including co-precipitation method, reverse micellar method, and hot injection method. Of these three methods, only the hot injection method produced an immediately viable product for application in the hybrid light emitting diode proposed. The co-precipitation method did generate the ability to perform the reverse micellar method which produced $\sim 30 \mathrm{~nm}$ diameter nanorods of mostly amorphous material. These rods were analyzed using XRD, and TEM. The hot injection method using octadecene as the solvent and various ligands, including oleic acid, benzene-1,4-dithiol, mercapto-benzoic acid and TOP, created quantum dots exhibiting photoluminescence in the green region of the visible spectrum. These quantum dots were characterized using UV-Visible Spectroscopy and Photoluminescence Spectroscopy. The oleic acid capped QD had a characteristic wavelength of $560 \mathrm{~nm}$. While the mercapto-benzoic acid and benzene-1,4-dithiol capped QD had a characteristic wavelengths of $510 \mathrm{~nm}$ and $555 \mathrm{~nm}$, respectively, all under the same operating conditions. For the purposes of device fabrication, the NN-Labs QDs
\end{abstract}


were mostly used because their performance was much better than quantum dots synthesized in house.

The fabrication of a hybrid light emitting diode has been developed with the structure ITO/PEDOT:PSS/PVK/CdSe QD/Alq3/Al. The device has exhibited a turn-on voltage from 2-3 V with currents ranging from 4-5 mA. The PEDOT:PSS layer was deposited using spin coating and analyzed using Photoluminescence Spectroscopy, Atomic Force Microscopy, Optical Microscopy, and Surface Profiling. The layer produce is sufficient for device fabrication. To facilitate proper hole transport and a sufficient quantum dot layer, a PVK/QD composite solution was used to create a monolayer layer of quantum dots by phase separation of the solutes in the solution. Alq3 and Al were deposited using thermal evaporation techniques. 


\section{TABLE OF CONTENTS}

ACKNOWLEDGEMENTS .............................................................................. iv

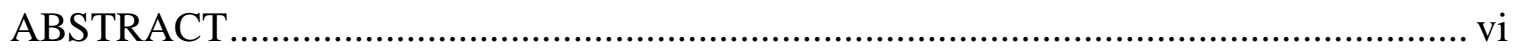

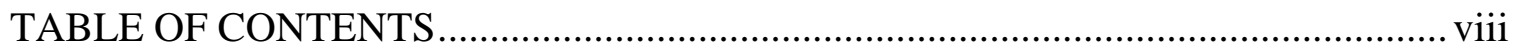

NOMENCLATURE ......................................................................................

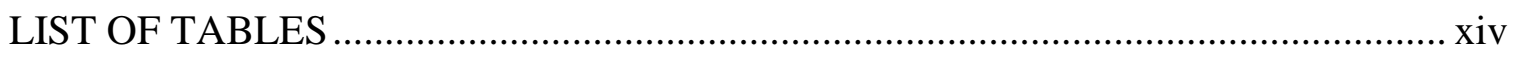

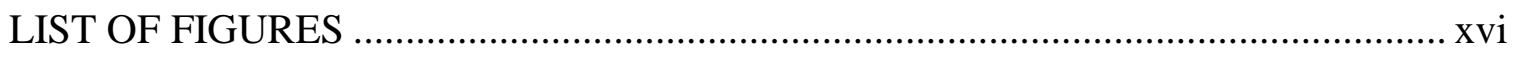

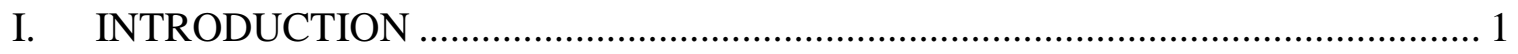

1.1 Semiconducting Nanoparticles - Quantum Dots ........................................... 1

1.2 Hybrid Light Emitting Diodes................................................................... 4

II. SYNTHESIS AND CHARACTERIZATION OF CADMIUM SELENIDE

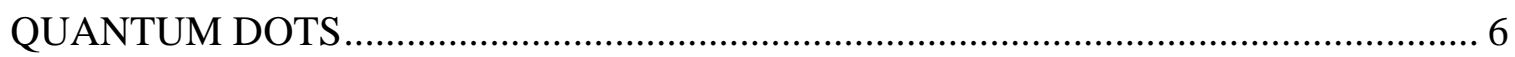


2.1 Introduction

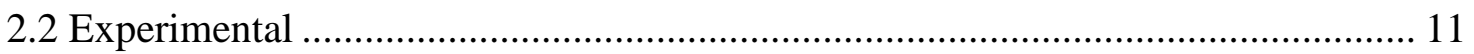

2.2.1 Co-precipitation Method of Quantum Dot Synthesis .................................... 11

2.2.2 Reverse Micellar Method of Quantum Dot Synthesis .................................. 12

2.2.3 Hot Injection Method of Quantum Dot Synthesis ...................................... 14

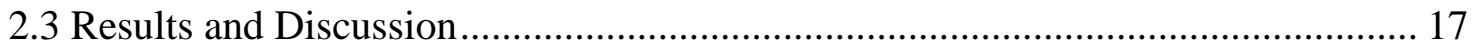

2.3.1 Co-precipitation Quantum Dot Synthesis Method ..................................... 17

2.3.2 Reverse Micellar Quantum Dot Synthesis .............................................. 20

2.3.3 Hot Injection Method of Quantum Dot Synthesis ..................................... 24

III. DEVELOPMENT OF QUANTUM DOT LIGHT EMITTING DIODES ........... 34

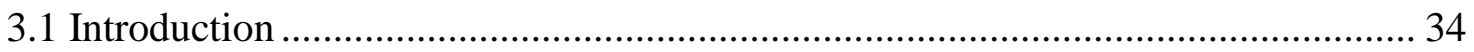

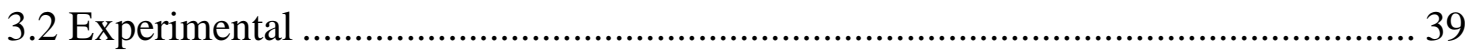

3.2.1 Preparation of Conductive Slides.......................................................... 39

3.2.2 Deposition of Polymer Layer................................................................ 39

3.2.3 Deposition of Quantum Dot Layer via Spin Coating.................................. 41

3.2.4 Deposition of Quantum Dot Layer via Inkjet Printing ................................ 41

3.2.5 Deposition of Quantum Dot Layer via Drop Casting .................................. 45

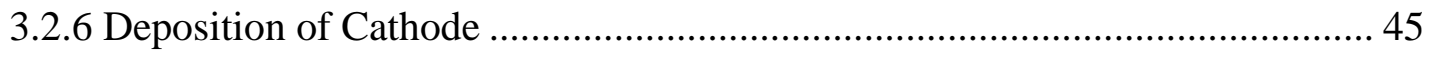

3.2.7 Device Testing and I-V Curve Generation ............................................. 49

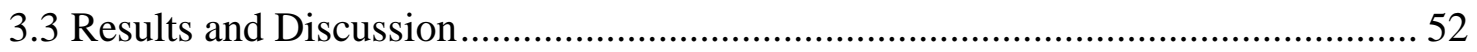


3.3.1 Polymer Layer Deposition and CharacterizationError! Bookmark not defined.

3.3.2 Deposition of Cathode Error! Bookmark not defined.

3.3.4 Quantum Dot Layer Deposition using Spin Coating and Devices Error!

Bookmark not defined.

3.3.5 Quantum Dot Layer Deposition using Inkjet Printing and Devices 78

3.3.6 Quantum Dot Layer Deposition using Drop Casting and Devices 80

IV. CONCLUSIONS AND FUTURE WORK ................................................... 82

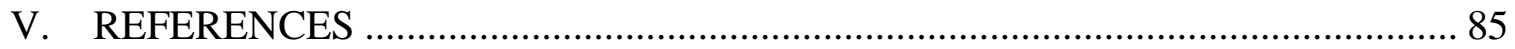

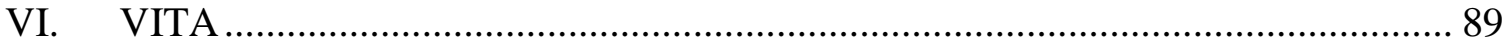


NOMENCLATURE

$\begin{array}{ll}\text { QDs } & \text { Quantum Dots } \\ \mathrm{ZnS} & \text { Zinc Sulfide } \\ \mathrm{CdSe} & \text { Cadmium Selenide } \\ \mathrm{eV} & \text { Electron Volts } \\ \mathrm{E} & \text { Energy (eV) } \\ \mathrm{d} & \text { diameter } \\ \hbar & \text { Planck's Constant } \\ \mathrm{m}^{*}{ }_{\mathrm{e}} & \text { effective mass of an electron } \\ \mathrm{m}^{*}{ }_{\mathrm{h}} & \text { effective mass of a hole } \\ \epsilon & \text { dielectric constant } \\ \mathrm{TEM} & \text { Transmission Electron Microscopy } \\ \text { LED } & \text { Light Emitting Diode }\end{array}$




\begin{tabular}{|c|c|}
\hline QD-LED & Quantum Dot Light Emitting Diode \\
\hline PV SC & Photovoltaic Solar Cells \\
\hline AOT & Dioctyl sulfosuccinate sodium salt \\
\hline UV & Ultraviolet \\
\hline XRD & X-Ray Diffraction \\
\hline PL & Photoluminescence Spectroscopy \\
\hline TOP & trioctylphosphine \\
\hline FWHM & Full Width Half Maximum \\
\hline EIL & Electron Injection Layer \\
\hline ETL & Electron Transport Layer \\
\hline HBL & Hole Blocking Layer \\
\hline ITO & Indium tin oxide \\
\hline PEDOT:PSS & Poly(3,4-ethylenedioxythiophene) Polystyrene sulfonate \\
\hline IPA & Isopropyl Alcohol \\
\hline CVD & Chemical Vapor Depostion \\
\hline $\mathrm{rpm}$ & Revolutions per minute \\
\hline $\mathrm{cP}$ & Centipoise \\
\hline $\mathrm{V}$ & Volts \\
\hline A & Amperes \\
\hline $\mathrm{Hz}$ & Hertz \\
\hline K & Sputtering Coating Constant \\
\hline I & Current \\
\hline $\mathrm{t}$ & time \\
\hline
\end{tabular}




\begin{tabular}{|c|c|}
\hline AFM & Atomic Force Microscopy \\
\hline $\mathrm{R}_{\mathrm{a}}$ & Average Surface Roughness \\
\hline $\mathrm{R}_{\mathrm{q}}$ & Root Mean Square Surface Roughness \\
\hline $\mathrm{R}_{\mathrm{pv}}$ & Maximum height Profile \\
\hline $\mathrm{R}_{\mathrm{sk}}$ & Skewness of the Surface Roughness \\
\hline $\mathrm{R}_{\mathrm{ku}}$ & Kurtosis of the Surface Roughness \\
\hline $\mathrm{P}$ & Pressure \\
\hline $\mathrm{x}$ & Mole \% in Liquid Phase \\
\hline $\mathrm{y}$ & Mole \% in Gas Phase \\
\hline$\gamma$ & Activity Coefficient \\
\hline $\mathrm{r}$ & Chain Length of Polymer \\
\hline$\Phi$ & Volume Fraction of Polymer \\
\hline$\chi$ & Flory-Huggins interaction parameter \\
\hline$\delta$ & Hildebrand solubility parameter \\
\hline LCST & Lower Critical Solution Temperature \\
\hline UCST & Upper Critical Solution Temperature \\
\hline $\mathrm{G}$ & Gibbs Free Energy \\
\hline $\mathrm{H}$ & Enthalpy \\
\hline $\mathrm{S}$ & Entropy \\
\hline$\Omega$ & Ohm \\
\hline $\mathrm{CP}$ & Conjugated Polymer \\
\hline PVK & Poly(9-vinylcarbazole) \\
\hline Alq3 & Tris(8-hydroxyquinolinato)aluminium \\
\hline
\end{tabular}




\section{LIST OF TABLES}

Table 1: Reference between Color, Wavelength, Diameter and Energy of Band Gap....... 3

Table 2: Materials used in Co-precipitation Quantum Dot Synthesis ........................... 11

Table 3: Micellar Solution Composition for Quantum Dot Synthesis........................... 13

Table 4: 90Plus Particle Size Testing Parameters...................................................... 13

Table 5: 90Plus Particle Analyzer Micellar Results .................................................. 14

Table 6: Substance used in Hot Injection Quantum Dot Synthesis .............................. 15

Table 7: Variations of Cadmium Precursor Solution for Hot Injection Quantum Dot

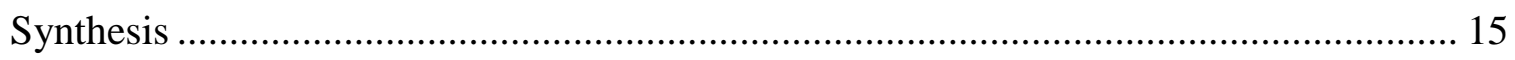

Table 8: Variations of Selenium Precursor Solution for Hot Injection Quantum Dot

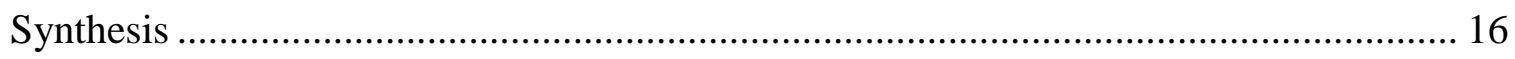

Table 9: Luminescent Properties Summary of Oleic Acid/TOP capped CdSe ............... 25 
Table 10: Luminescent Properties of Oleic Acid/TOP capped CdSe with increased concentration by one order of magnitude

Table 11: Luminescent Properties Summary of mercapto-benzoic acid/TOP capped CdSe

QD

Table 12: Luminescent Properties Summary of benzene-1,4-dithiol/TOP capped CdSe

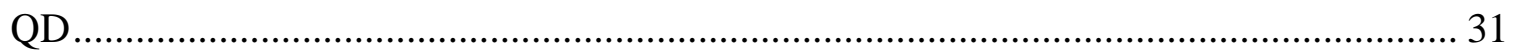

Table 13: Luminescent Properties of NN-Labs Quantum Dots..................................... 33

Table 14: Valence and Conduction band energies for some commonly used organics of

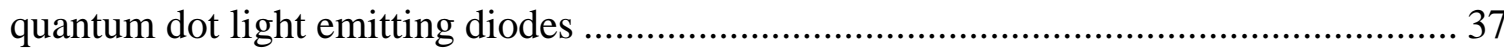

Table 15: Work function of commonly used anode and cathode materials in quantum dot

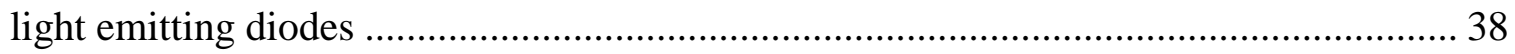

Table 16: AFM Analysis of 50\% Ethanol in PEDOT:PSS Solution Spin Coated........... 56

Table 17: AFM Analysis of 50\% Ethanol in PEDOT:PSS Solution - Smaller Area....... 57

Table 18: AFM Analysis of Chemical Vapor Deposited PEDOT:TAA.......................... 57

Table 19: AFM Analysis of Chemical Vapor Deposited PEDOT:TAA - Smaller Area .. 58 


\section{LIST OF FIGURES}

Figure 1: TEM image of a CdSe QDs synthesized using the hot injection method [3]..... 3 Figure 2: TEM images of CdSe QDs at Water to Surfactant Molar Ratio of (a) 3 (b) 5 (c)

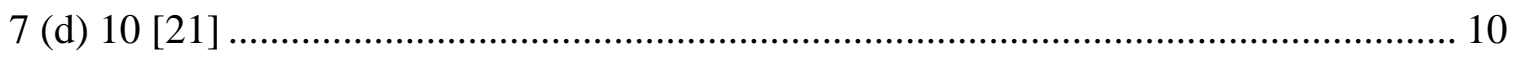

Figure 3: XRD Analysis of Co-precipitation Quantum Dot Synthesis before purification 18

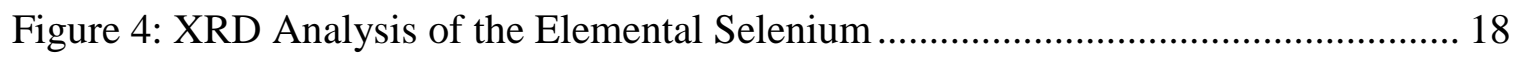

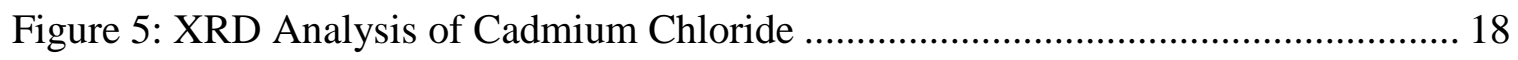

Figure 6: XRD Analysis of Purified CdSe Sample..................................................... 19

Figure 7: TEM Images of Co-precipitation Quantum Dot Synthesis with Scale Bars of

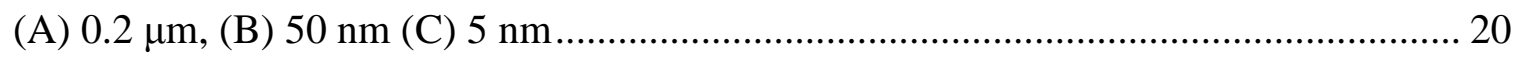

Figure 8: UV-visible Spectroscopy of Blank Micellar Solution and Reacted Micellar

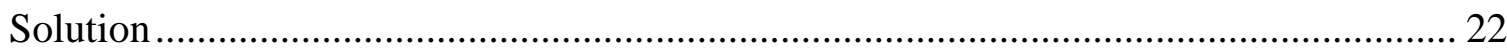


Figure 9: XRD Analysis of the Dried Synthesis Product

Figure 10: TEM Images of the Reverse Micellar Synthesis Method with scale bars of (A)

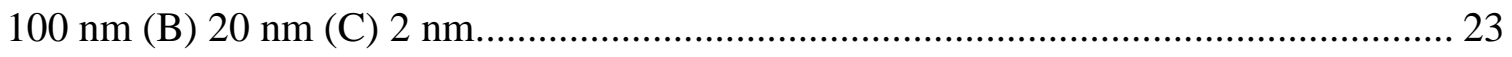

Figure 11: Photoluminescence Spectroscopy of Oleic Acid/TOP capped CdSe .............. 25

Figure 12: XRD of Oleic Acid/TOP capped CdSe .................................................. 26

Figure 13: Image of Oleic Acid/TOP capped CdSe under UV Torch ........................... 26

Figure 14: Photoluminescence Spectroscopy of Oleic Acid/TOP capped CdSe with increased concentration by one order of magnitude .............................................. 28

Figure 15: PL Spectroscopy of mercapto-benzoic acid/TOP capped CdSe QD .............. 30

Figure 16: XRD of mercapto-benzoic acid/TOP capped CdSe QD ............................. 30

Figure 17: Photoluminescence Spectroscopy of benzene-1,4-dithiol/TOP capped CdSe

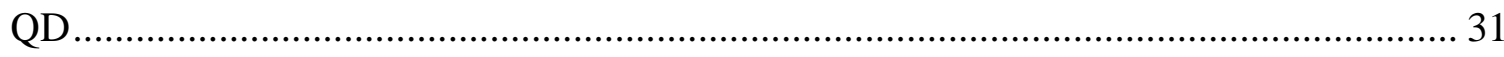

Figure 18: XRD of benzene-1,4-dithiol/TOP capped CdSe QD................................... 32

Figure 19: Photoluminescence Spectroscopy of Quantum Dots purchased from NN-Labs

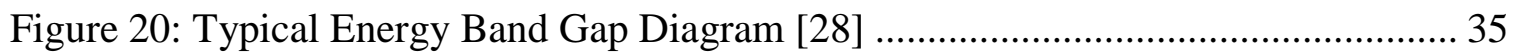

Figure 21: Proposed Device Structure of Hybrid LED; (A) 3-D General Depiction of Device; (B) Proposed Materials and Energy Band Gap Diagram of Device................... 36

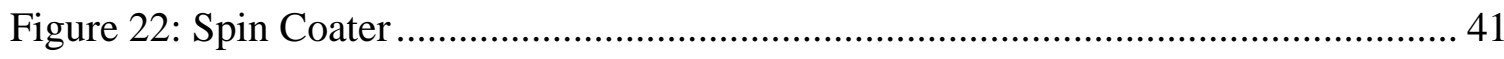

Figure 23: Dimatix Materials Printer DMP-2800 Series .......................................... 42

Figure 24: Anton Paar AMVn Automated Microviscometer ...................................... 43

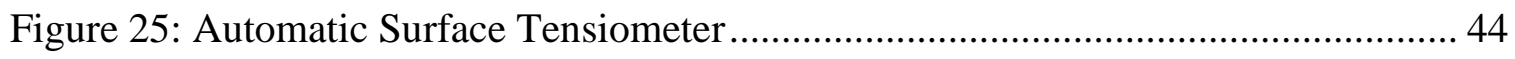

Figure 26: SPI-Model Sputter Coater Model 11430 ................................................... 46 
Figure 27: Example of Sputtering Process.................................................................... 47

Figure 28: Mask for Thermal Evaporator ...................................................................... 48

Figure 29: Examples of Aluminum Deposited onto Sample Device............................... 48

Figure 30: Thermal Evaporator.................................................................................... 49

Figure 31: LabView 7.1 Graphical Interface for Device Testing .................................... 49

Figure 32: Photograph of Completed Device ……………...................................... 50

Figure 33: Photograph of Probing Station \#1 …………................................................ 51

Figure 34: Photograph of Probing Station \#2 ……………......................................... 51

Figure 35: 10\% Ethanol in PEDOT:PSS Soltuion Spin Coated on ITO........................... 53

Figure 36: 30\% Ethanol in PEDOT:PSS Solution Spin Coated on ITO........................... 53

Figure 37: 50\% Ethanol in PEDOT:PSS Solution Spin Coated on ITO.......................... 53

Figure 38: Chemical Vapor Deposited PEDOT Layer on ITO......................................... 55

Figure 39: AFM Image of 50\% Ethanol in PEDOT:PSS Solution Spin Coated on ITO

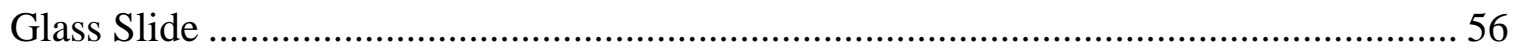

Figure 40: 3-D AFM Depiction of Chemical Vapor Deposited PEDOT:TAA ................. 57 Figure 41: Spin Coated PEDOT:PSS - mercapto-benzoic acid/TOP capped CdSe QD

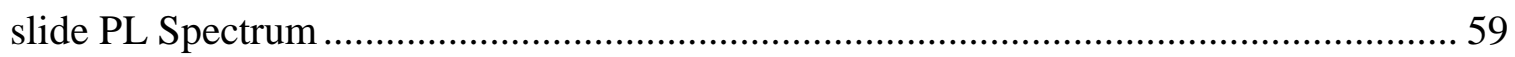

Figure 42: CVD PEDOT:TAA - mercapto-benzoic acid/TOP capped CdSe QD slide PL

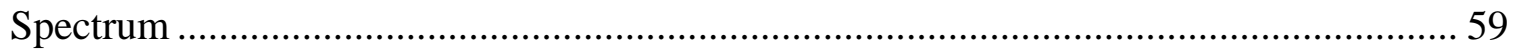

Figure 43: Schematic phase diagram for solutions of a molecularly uniform polymer. Polymer lean phase (sol): A - stable, B - metastable, C - unstable and segregation of a gel phase. Polymer rich phase (gel): D - stable, E - metastable, F - unstable and

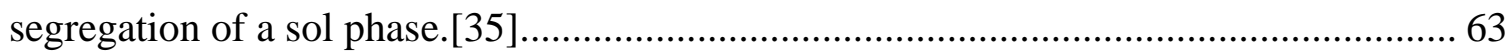


Figure 44: (Left) Depiction of the crystallization of a thermoreversible gel [39] (Right)

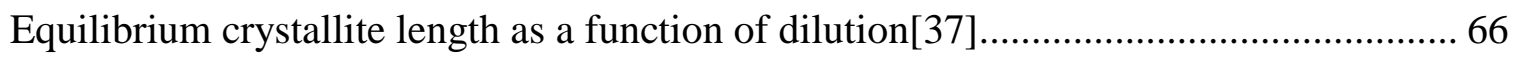

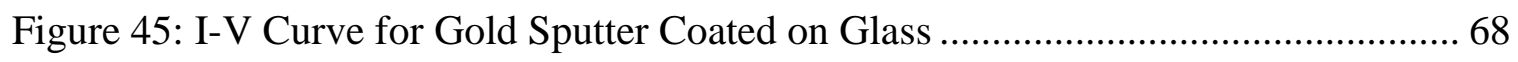

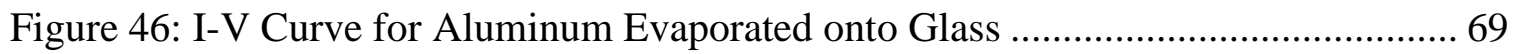

Figure 47: PL Mapping of Spin Coated Device..................................................... 70

Figure 48: PL Spectroscopy of the Spin Coated Device........................................... 70

Figure 49: Photograph of Spin Coated Device under UV irradiation............................ 71

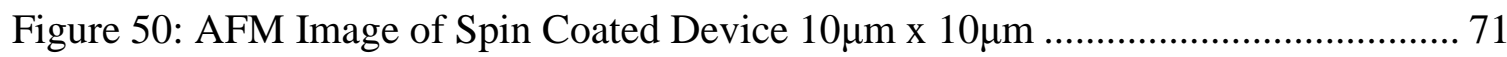

Figure 51: AFM Image of Spin Coated Device 100nm x 100nm................................ 71

Figure 52: Run 1 of a Spin Coated Original Device Structure .................................. 72

Figure 53: Run 2 of a Spin Coated Original Device Structure .................................... 73

Figure 54: Optical Images of PVK/QD spin coated layer 100 $\mu \mathrm{m}$ Scale Bar.................. 74

Figure 55: I-V Curve for a PVK/QD Spin Coated Device 1 .................................... 77

Figure 56: I-V Curve for a PVK/QD Spin Coated Device 2 ................................... 77

Figure 57: Device with ITO/PEDOT:PSS/PVK/QDs/Alq3/Al ................................. 78

Figure 58: Photograph of an inkjet printed quantum dot layer .................................. 79

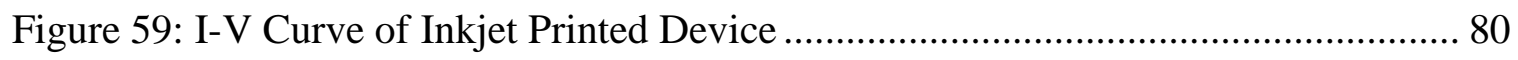

Figure 60: I-V Curve of Drop Casted PVK/QD Device Run 1 ...................................... 81

Figure 61: I-V Curve of Drop Casted PVK/QD Device Run 1 ................................... 81

Figure 62: Proposed Device Structure for Future Work ........................................... 84 


\section{INTRODUCTION}

\subsection{Semiconducting Nanoparticles - Quantum Dots}

Quantum dots (QDs) come in different shapes of various materials but are characterized by their size range. They typically range from 1 to $10 \mathrm{~nm}$ and are made of semiconducting materials, such as, $\mathrm{ZnS}, \mathrm{CdSe}$, and even Carbon. What differentiates these particles from bulk materials is its size which affects its optical characteristics. The optical properties are directly related to the band gap of the material. The band gap of a material is the energy difference between the valence band and the conduction band of a material. By controlling the size of the particle, the electrons and holes are confined leading to an increase in its band gap. This effect is referred to as quantum confinement.[1]

The size of the particle which must be met in order to start to exhibit these characteristics is the Bohr's Radius of an exciton. Bohr's Radius of an exciton is the 
distance between the atom's hole and electron, or its exciton pair and, varies for each semiconductor crystal. [5] The Bohr's radius of an exciton differs from the Bohr's radius of a material by how the particle is considered. In reference to an exciton, the nanoparticle is represented the same way as an individual atom. The band gap is increased as the size of the particle decreases. There have been calculations done to predict the amount of change in the band gap based on the diameter of the particle. One such equation is showcased in Equation 1.

$$
E^{*} \approx E_{g}+\frac{\hbar^{2} \pi^{2}}{2 R^{2}}\left(\frac{1}{m_{e}}+\frac{1}{m_{h}}\right)-\frac{1.8 e^{2}}{\varepsilon R}
$$

The above equation calculates the band gap energy of a crystal, $\mathrm{E}^{*}$, based on radius and energy band gap of the bulk material, $\mathrm{E}_{\mathrm{g}}$. $\hbar$ is Planck's Constant, $r$ is the radius, $\epsilon$ is the dielectric constant, $m_{e}$ and $m_{h}$ is the effective mass of the electron and hole, respectively. Although this equation does not accurately predict the energy band gap at the nanoscale because it assumes that the crystal's potential is a spherical well of infinite depth, it does show how the band gap depends on size when the Schrödinger Equation is solved.

In the case of CdSe with a bulk band gap of $1.74 \mathrm{eV}$ and decreasing the size of the molecule, the wavelength of the light emitted when an electron relaxes into the valence band can be altered across the entire visible spectrum. A reference table for wavelength, diameter as calculated from the above equation and energy in $\mathrm{eV}$ is provided in Table 1. The band gap of bulk CdSe is in the near-infrared region on the light spectrum, making it ideal for increasing its band gap energy into the visible spectrum. 
Table 1: Reference between Color, Wavelength, Diameter and Energy of Band Gap

\begin{tabular}{|l|l|l|l|}
\hline Color & Wavelength & Energy & Diameter* \\
\hline Red & $700 \mathrm{~nm}$ & $1.771 \mathrm{eV}$ & $22 \mathrm{~nm}$ \\
\hline Reddish Orange & $650 \mathrm{~nm}$ & $1.909 \mathrm{eV}$ & $9.36 \mathrm{~nm}$ \\
\hline Orange & $600 \mathrm{~nm}$ & $2.067 \mathrm{eV}$ & $6.75 \mathrm{~nm}$ \\
\hline Yellow & $580 \mathrm{~nm}$ & $2.138 \mathrm{eV}$ & $6.11 \mathrm{~nm}$ \\
\hline Yellowish Green & $550 \mathrm{~nm}$ & $2.254 \mathrm{eV}$ & $5.38 \mathrm{~nm}$ \\
\hline Green & $500 \mathrm{~nm}$ & $2.480 \mathrm{eV}$ & $4.48 \mathrm{~nm}$ \\
\hline Blue & $450 \mathrm{~nm}$ & $2.765 \mathrm{eV}$ & $3.81 \mathrm{~nm}$ \\
\hline Violet & $400 \mathrm{~nm}$ & $3.100 \mathrm{eV}$ & $3.30 \mathrm{~nm}$ \\
\hline
\end{tabular}

*Calculated from Equation [1]

The most common way of synthesizing QDs is the hot injection method. This method involves the use of high boiling point solvents, solutions of quantum dot materials, for example, $\mathrm{Cd}$ and Se solutions, in the presence of a ligand. The two precursor solutions are mixed and, as the crystals nucleate, the ligand stops the growth of the crystals at a small size. [6] Figure 1 displays a typical transmission electron microscope (TEM) image of a QD.

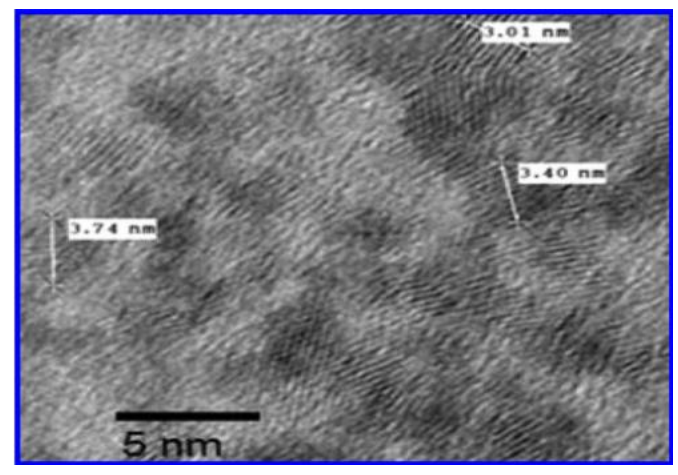

Figure 1: TEM image of a CdSe QDs synthesized using the hot injection method [2]

The reason for using QDs as an emissive layer is because of the QDs potential to emit one photon for every exciton generated resulting in $100 \%$ internal quantum efficiency. When compared with other conventional devices such as InGaN/GaN LEDs with an internal quantum efficiency of $70 \%$ [3], the use of quantum dots becomes quite lucrative. In comparison to organic based emissive layers, the theoretical internal quantum 
efficiency is limited to $25 \%$ because the ratio of singlet to triplet electron states is $1: 3$. Although, for phosphorescent organic materials it has been found the $>25 \%$ internal quantum efficiency can be produce due to spin-orbit coupling. [4]

There are multiple avenues for obtaining quantum dots today. There are companies that synthesis high quality, well known varieties of quantum dots in different solvents. The other method involves producing the quantum dots in the lab. The primary purpose of synthesizing quantum dots was to have complete control of the design of the quantum dot from composition to passivation shell so as to provide better functionalization in a quantum dot light emitting diodes (QD-LED). The secondary reason for developing quantum dot synthesis expertise was to possibly innovate the way quantum dots are synthesized. In this thesis, new methods for quantum dot synthesis were explored and refined including a co-precipitation method, a reverse micellar growth method and conventional hot-injection methods.

\subsection{Hybrid Light Emitting Diodes}

In recent years, there has been much research in Light Emitting Diodes (LED) and photovoltaic solar cells (PV SC). This research involves the use of semiconducting materials to emit and capture photons. The first use of an inorganic semiconductor as an LED was in 1907 with SiC by H.J. Round [5] and LEDs have come a long way since then. While creating an inorganic LED, various types of doping at different amounts of other semiconducting metals can be used. This method allows for epitaxial growth of different layers of the device, decreasing the amount of defects which contribute to charge traps. Charge traps contribute negatively to efficiency by increasing non-radiative 
recombination, such as, phonons.[6] There must be different layers in the device to facilitate a radiative combination of an electron-hole pair, a.k.a. an exciton.

Since conjugated polymers were discovered by Alan J. Heegar, Alan G. MacDiarmid and Hideki Shirakawa in 1977, LEDs have branched into the new field of organic devices.[7] Organic Light Emitting Diodes (OLED) and Polymer Light Emitting Diodes (PLED) have the capability of creating the flexible, light weight efficient lighting of the future.[8] Although OLEDs have the promise of surpassing conventional LEDs in performance, OLED materials and manufacturing are not advanced enough to provide this economically. To facilitate this change and manage the weaknesses of both inorganic and organic LEDs, hybrid devices are now of particular interest. By developing a hybrid device, manufacturing cost can potentially be decreased, durability can be increased, and efficiency can be increased.

In this thesis, a hybrid device is attempted by combining quantum dots with conjugated polymers to create a QD-LED. The ultimate process of fabricating the device should involve the use of inkjet printing as it is easily scalable and versatile. The proposed structure of the hybrid LED is ITO/PEDOT:PSS/CdSe QDs/Au. One of the highlights of this structure is the increased simplicity over conventional OLEDs. Conventional OLEDs involve a multitude of layers which include carrier transport, injection, and blocking to facilitate a radiative recombination of an electron-hole pair. The other major highlight of this structure is its solution fabrication which would be deposited via inkjet printing. 


\section{SYNTHESIS AND CHARACTERIZATION OF CADMIUM SELENIDE QUANTUM DOTS}

\subsection{Introduction}

The key to synthesizing quantum dots is to create homogeneous nucleation and inhibit the growth of nucleates at 100-10,000 atoms. To inhibit the growth, a small chained organic molecule is generally used to form a chelate-type bond with the nanocrystals. [9] Although not the first to synthesis what has come to be known as quantum dots, Bawendi, Murray and Norris were the one's credited with developing the hot injection method in the paper, Synthesis and Characterization of Nearly Monodisperse $\mathrm{CdE}(\mathrm{E}=\mathrm{S}$. Se, Te). [10] This method has become the standard for producing quantum dots of a narrow distribution and high quantum yield while having the ability to control the characteristic wavelength. Unfortunately, the hot injection method involves the use of highly hazardous materials and dangerous operating 
conditions in a highly sensitive environment. In order to develop a better understanding of the mechanisms involved in quantum dot synthesis, the hot injection method was pursued. This serves as a starting point from which to base future synthetic routes and improvements.

One synthesis step which can significantly increase a quantum dot's fluorescence is an inorganic passivation shell. These types of quantum dots are referred to as core-shell quantum dots. A passivation shell is used to reduce the number of dangling bonds. The ligand acts as a type of passivation layer to stop the growth of the nanocrystals while an inorganic passivation shell provides surface passivation which reduces surface charge traps and helps confine excitons through use of its band gap energy. One system has been studied extensively, CdSe/ZnS. [11] The present work on quantum dot synthesis study did not incorporate the core-shell approach primarily to focus on QD-LED device fabrication.

There are three things that affect the formation of nanocrystals, the concentrations of the precursor solutions, the solvent used, and the ligand used. It has been shown that the solvent used can affect the type of crystalline structure formed. [12] The temperature of the synthesis does have an effect on the ability to form quantum dots but depending on the system, quantum dots can be synthesized at temperatures from room temperature to $300^{\circ} \mathrm{C}$. The temperature of the synthesis may affect the quantum yield of the quantum dot which is the amount of photons created from excitons and directly related to its fluorescence. In experiments with $\mathrm{ZnS}$ shells performed at temperatures from $65^{\circ} \mathrm{C}$ to $180^{\circ} \mathrm{C}$, quantum yields were reported to be $26 \%$ to $46 \%$. [13] While in similar experiments where the temperature was at $245^{\circ} \mathrm{C}$, the quantum yield was as high as $93 \%$. 
[14] The concentrations of the precursors need to be sufficiently dilute for the development of a monodisperse solution of nanocrystals. In this study, the effect of concentration on nanocrystal size will be observed. The concentration of the ligand is important and needs to be high enough to cover the surface of the quantum dots. Consequently, it needs to be in excess. The excessive use of the ligand does not negatively affect the synthesis of the quantum dot but does significantly affect subsequent steps after nucleation, such as the formation of a shell. [15]

The co-precipitation method describes a technique which does not involve the use of organic solvents at high temperatures. This method used was based on a previous paper [16] which involved the use of two aqueous precursor solutions. The two solutions were then combined to form a CdSe solution. The procedure is detailed in the experimental section. Ultimately, this type of method could be used to lower synthesis costs and provide a safer environment for manipulating the quantum dots along with a possible use in biological applications.

The reverse micellar method spun off from the co-precipitation method as it provided an aqueous phase in the reverse micellar system. There have been minimal studies in the use of reverse micelle synthesis when compared to other solution techniques. One method involved the use of a surfactant containing one of the precursor materials, $\mathrm{Cd}$, followed by the second material, Sulfur, bubbled through the solution to form equilateral nano-triangles CdS with sides of $10 \mathrm{~nm}$. Unfortunately, it was reported that the particles were not stable as the surfactant readily desorb and the particles agglomerate. [17] 
The aqueous phase of the reverse micellar solution used in the reverse micellar synthesis of this study employed the two precursor solutions from the co-precipitation method. Two reverse micelle solutions were made, one holding the $\mathrm{Cd}$ aqueous solution and one holding the Se aqueous solution, and combined to form the CdSe product. This process was of particular interest because it offered a highly controllable environment with relatively low cost materials.

In literature, similar reverse micellar procedures involve the use of high temperatures which are essentially the same process as the hot injection method, [18] while two others are extremely similar to the method of this study both of which were being completed at the same time as this study. $[19,20]$ The use of AOT as the surfactant differentiated one of the papers. The reverse micellar system was then optimized based on $\mathrm{Cd}$ and $\mathrm{Se}$ concentrations in their respective solutions as well as surfactant concentration. The average diameter of the QDs ranged from 3.0 to 4.1 depending on the surfactant molar ratio and exhibited a peak quantum yield of $\sim 30 \%$. [20] TEM images are displayed in Figure 2, with (b) having the highest fluorescent performance. 


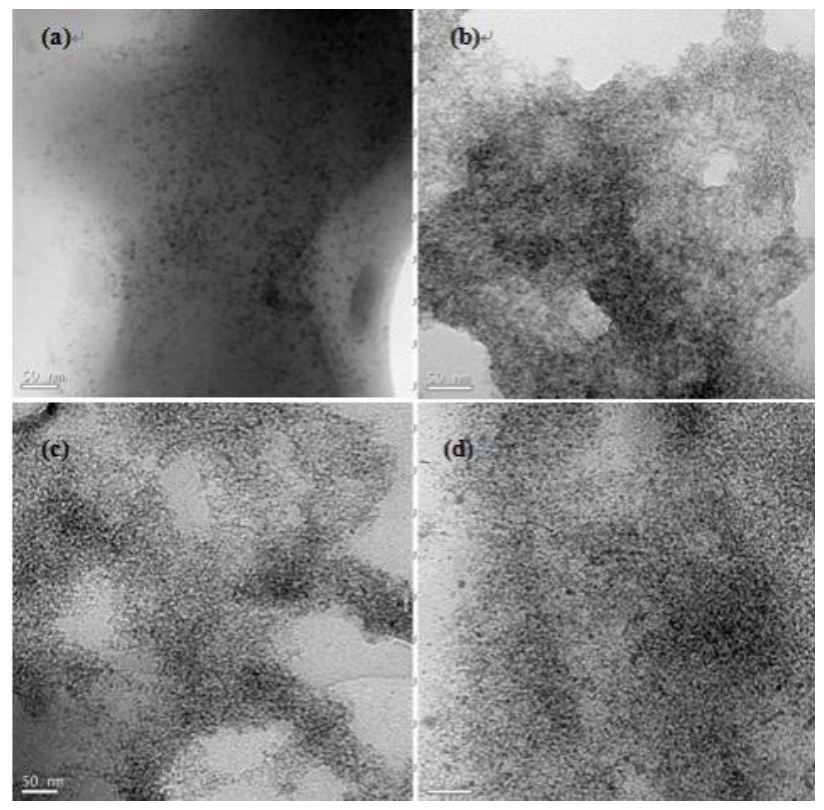

Figure 2: TEM images of CdSe QDs at Water to Surfactant Molar Ratio of (a) 3 (b) 5 (c) 7 (d) 10 [20] 


\subsection{Experimental}

The quantum dots prepared for application in the thin film device were developed using the hot injection method. Other methods were considered and attempted but did not yield viable results for applications in a light emitting diode. These methods included a reverse micellar method and a co-precipitation method which will be identified and the results discussed. For all experiments, the solution was illuminated with a UV light in a dark room for immediate qualitative evaluation of any photoluminescence properties.

\subsubsection{Co-precipitation Method of Quantum Dot Synthesis}

An aqueous co-precipitation method was pursued to generate quantum dots. This method involved the combination of two precursor solution, one cadmium solution and one selenium solution. The substances and concentrations used in this method are shown in Table 2.

Table 2: Materials used in Co-precipitation Quantum Dot Synthesis

\begin{tabular}{|c|c|c|c|c|}
\hline Material & Supplier & $\begin{array}{c}\text { Molecular } \\
\text { Weight }\end{array}$ & Purity & Concentration \\
\hline $\begin{array}{c}\text { Sodium } \\
\text { Borohydride } \\
\left(\mathrm{NaBH}_{4}\right)\end{array}$ & Sigma Aldrich & $37.83 \mathrm{~g} / \mathrm{mol}$ & $\geq 99 \%$ & $0.04 \mathrm{M}$ \\
\hline Selenium & Sigma Aldrich & $78.96 \mathrm{~g} / \mathrm{mol}$ & $99.99 \%$ & $0.016 \mathrm{M}$ \\
\hline $\begin{array}{c}\mathrm{Cadmium} \mathrm{Nitrate} \\
\left(\mathrm{Cd}\left(\mathrm{NO}_{3}\right)_{2} \cdot 4 \mathrm{H}_{2} \mathrm{O}\right)\end{array}$ & Alfa Aesar & $308.47 \mathrm{~g} / \mathrm{mol}$ & $99.9 \%$ & $0.016 \mathrm{M}$ \\
\hline
\end{tabular}

The elemental selenium as purchased by Sigma Aldrich was not soluble in water.

Therefore, the sodium borohydride was added to the selenium while in water under continuous flow of argon until completely dissolved which was approximately one hour in a round bottom flask. The flow of the argon was maintained at 5 cubic feet per minute. The argon flow was essential to prevent oxidation of the selenium. The concentration 
calculations were based on fully dissolved selenium. If selenium did not fully dissolve, the solution was sonicated using a sonicator bath for five minutes. The cadmium nitrate was soluble in water. The cadmium solution was then degased for one hour to not introduce any oxygen into the reaction.

The cadmium solution was then combined with the selenium solution under an argon flow. The two solutions were added by two methods, immediately combining the cadmium solution to the selenium and adding the cadmium solution dropwise to the selenium solution at rates from 1-3 drops per second. The resulting solution for both combination methods yielded a black precipitate which was analyzed using X-Ray Diffraction (XRD).

Purification of the sample from potential residual reactants was developed and completed. The method for extracting unreacted cadmium solution involved the washing of the sample with ethanol (Decon Labs, 200 Proof) three times followed by drying in the vacuum oven at $60^{\circ} \mathrm{C}$ and slight vacuum. Unreacted selenium and selenium oxide were removed from the sample using sodium borohydride at $0.04 \mathrm{M}$. This procedure involved the redispersion of the sample in water. Sodium borohydride was then added to the solution and allowed to react at temperatures up to $60^{\circ} \mathrm{C}$ and stirring for three hours. The solids were then centrifuged, washed with ethanol once, and dried in a vacuum oven at a temperature of $60^{\circ} \mathrm{C}$. The subsequent product was analyzed using XRD and TEM.

\subsubsection{Reverse Micellar Method of Quantum Dot Synthesis}

The first step in developing a reverse micellar synthesis process was optimizing a reverse micellar solution that has an appropriate micelle size. The surfactant chosen to 
form micelles was TritonX-100. The organic phase of the solution was n-octane with water being the medium in the micelles. An alcohol, 1-hexanol, was used to help stabilize the micelles. The initial composition formed an opaque solution which was then titrated with the 1-hexanol to form the micellar solution and find the optimal composition. The final solution composition is displayed in Table 3.

Table 3: Micellar Solution Composition for Quantum Dot Synthesis

\begin{tabular}{|c|c|c|c|}
\hline Substance & Volume \% & Supplier & Grade \\
\hline Triton X-100 & $11.4 \%$ & Alfa Aesar & $>99 \%$ \\
\hline n-Octane & $76.1 \%$ & Sigma Aldrich & $99 \%$ \\
\hline Water & $4.1 \%$ & Deionized & Type II \\
\hline 1-Hexanol & $8.4 \%$ & Alfa Aesar & $99 \%$ \\
\hline
\end{tabular}

The size of the micelles was analyzed using a Brookhaven 90Plus Particle Sizer. This method utilizes light scattering in the solution to differentiate between larger particles and small particles through fluctuations in decay times of interference of the scattered light. These fluctuations were correlated to the diffusion constants of the particles which are based on the size of the particles. By combining these correlations, an effective average diameter of the particles in the solution can be deduced. The sample was filtered using a $0.2 \mu \mathrm{m}$ nylon syringe filter and loaded into a glass cuvette at the measurement parameters given in Table 4. The results from the test are provided in Table 5.

Table 4: 90Plus Particle Size Testing Parameters

\begin{tabular}{|c|c|}
\hline Temperature & $20^{\circ} \mathrm{C}$ \\
\hline Viscosity & $0.54 \mathrm{cP}$ \\
\hline Ref. Index Fluid & 1.4 \\
\hline Angle & 90 \\
\hline Wavelength & $658.3 \mathrm{~nm}$ \\
\hline Dust Cutoff & 5 \\
\hline Runs Completed & 5 \\
\hline Run Duration & $0: 02: 00$ \\
\hline Total Elapsed Time & $0: 10: 00$ \\
\hline
\end{tabular}




\begin{tabular}{|c|c|}
\hline Average Count Rate & $142.4 \mathrm{kcps}$ \\
\hline Ref. Index Real & 1.492 \\
\hline
\end{tabular}

Table 5: 90Plus Particle Analyzer Micellar Results

\begin{tabular}{|c|c|c|c|c|}
\hline Run & Effective Diameter & Half Width & Polydispersity & Baseline Index \\
\hline $\mathbf{1}$ & $28.4 \mathrm{~nm}$ & $13.3 \mathrm{~nm}$ & 0.22 & $0.4 / 12.15 \%$ \\
\hline $\mathbf{2}$ & $29.4 \mathrm{~nm}$ & $13.3 \mathrm{~nm}$ & 0.205 & $0.0 / 15.88 \%$ \\
\hline $\mathbf{3}$ & $29.9 \mathrm{~nm}$ & $13.7 \mathrm{~nm}$ & 0.211 & $0.0 / 13.93 \%$ \\
\hline $\mathbf{4}$ & $30.5 \mathrm{~nm}$ & $14.6 \mathrm{~nm}$ & 0.229 & $0.0 / 12.50 \%$ \\
\hline $\mathbf{5}$ & $31.2 \mathrm{~nm}$ & $14.6 \mathrm{~nm}$ & 0.219 & $0.0 / 15.67 \%$ \\
\hline Mean & $29.9 \mathrm{~nm}$ & $13.9 \mathrm{~nm}$ & 0.217 & $0.1 / 14.03 \%$ \\
\hline Std. Error & $0.5 \mathrm{~nm}$ & $0.3 \mathrm{~nm}$ & 0.004 & $0.1 / 0.78$ \\
\hline
\end{tabular}

The next step of this procedure involved the creation of the two precursor solutions, cadmium solution and the selenium solution. The materials used and concentrations in this step are the same as in Table 2. The concentration of the materials were: sodium borohydride - 0.5 M; selenium - 0.1 M; Cadmium Nitrate - $0.1 \mathrm{M}$. The aqueous solutions of selenium and cadmium were required for the precursor reverse micellar solutions. The solutions were created using the same methodology as in the coprecipitation method.

The two solutions were than combined under stirring and refluxed for 3 hours to produce a red solution. The red solution was then analyzed using UV-Visible Spectroscopy. The particles were extracted from the solution by drying and the resulting product was a black solid. The black solid was analyzed using XRD and TEM.

\subsubsection{Hot Injection Method of Quantum Dot Synthesis}

The Hot Injection Method refers to the process of combining an organic selenium solution to a heated organic cadmium solution. The components of the selenium solution include a selenium source, a dissolving agent, and the solvent. The components of the 
cadmium solution include a cadmium source, a capping ligand, and the solvent. The various substances used are showcased in Table 6 . The source of cadmium and selenium were the same as the previous synthesis methods, found in Table 2. The synthesis procedure was optimized by altering the concentrations of the reactants. Table 8 and 9 contain the variations of the cadmium and selenium solution, respectively.

Table 6: Substance used in Hot Injection Quantum Dot Synthesis

\begin{tabular}{|c|c|c|c|c|}
\hline Purpose & Material & Supplier & Molecular Weight & Purity \\
\hline $\begin{array}{c}\text { Capping Ligand } \\
\text { /Dissolving Agent }\end{array}$ & $\begin{array}{c}\text { Tri-n-octylphosphine } \\
\text { (TOP) }\end{array}$ & Alfa Aesar & $308.47 \mathrm{~g} / \mathrm{mol}$ & $90 \%$ \\
\hline Solvent & Cyclohexanol & Alfa Aesar & $100.16 \mathrm{~g} / \mathrm{mol}$ & $99 \%$ \\
\hline Solvent & 1-Octadecene & Alfa Aesar & $252.48 \mathrm{~g} / \mathrm{mol}$ & $90 \%$ \\
\hline Capping Ligand & Oleic Acid & Alfa Aesar & $282.47 \mathrm{~g} / \mathrm{mol}$ & $90 \%$ \\
\hline Capping Ligand & benzene-1,4-dithiol & $\begin{array}{c}\text { Sigma } \\
\text { Aldrich }\end{array}$ & $142.24 \mathrm{~g} / \mathrm{mol}$ & $99 \%$ \\
\hline Capping Ligand & mercapto-benzoic acid & $\begin{array}{c}\text { Sigma } \\
\text { Adlrich }\end{array}$ & $154.19 \mathrm{~g} / \mathrm{mol}$ & $99 \%$ \\
\hline
\end{tabular}

Table 7: Variations of Cadmium Precursor Solution for Hot Injection Quantum Dot Synthesis

\begin{tabular}{|l|l|l|l|}
\hline Solvent & $\begin{array}{l}\text { Cadmium } \\
\text { Concentration }\end{array}$ & Capping Ligand & $\begin{array}{l}\text { Capping Ligand } \\
\text { Concentration }\end{array}$ \\
\hline 1-Octadecene & $0.2 \mathrm{M}$ & Oleic Acid & $5.3 \%$ by Volume \\
\hline & $20 \mathrm{mM}$ & Oleic Acid & $5.3 \%$ by Volume \\
\hline & $2 \mathrm{mM}$ & benzene-1,4-dithiol & $2 \mathrm{mM}$ \\
\hline & & mercapto-benzoic acid & $2 \mathrm{mM}$ \\
\hline & & Oleic Acid & $5.3 \%$ by Volume \\
\hline Cyclohexanol & $0.2 \mathrm{M}$ & None & N/A \\
\hline & $0.1 \mathrm{M}$ & Oleic Acid & $5.3 \%$ by Volume \\
\hline
\end{tabular}


Table 8: Variations of Selenium Precursor Solution for Hot Injection Quantum Dot Synthesis

\begin{tabular}{|l|l|l|l|}
\hline Solvent & $\begin{array}{l}\text { Selenium } \\
\text { Concentration }\end{array}$ & Dissolving Agent & $\begin{array}{l}\text { Dissolving Agent } \\
\text { Concentration }\end{array}$ \\
\hline 1-Octadecene & $2 \mathrm{M}$ & $\begin{array}{l}\text { Tri-n-octylphosphine } \\
\text { (TOP) }\end{array}$ & $\begin{array}{l}10 \% \text { by volume of } \\
\text { Selenium Solution }\end{array}$ \\
\hline & $200 \mathrm{mM}$ & $\begin{array}{l}\text { Tri-n-octylphosphine } \\
\text { (TOP) }\end{array}$ & $\begin{array}{l}10 \% \text { by volume of } \\
\text { Selenium Solution }\end{array}$ \\
\hline & $20 \mathrm{mM}$ & $\begin{array}{l}\text { Tri-n-octylphosphine } \\
\text { (TOP) }\end{array}$ & $\begin{array}{l}10 \% \text { by volume of } \\
\text { Selenium Solution }\end{array}$ \\
\hline Cyclohexanol & $2 \mathrm{M}$ & $\begin{array}{l}\text { Tri-n-octylphosphine } \\
\text { (TOP) }\end{array}$ & $\begin{array}{l}10 \% \text { by volume of } \\
\text { Selenium Solution }\end{array}$ \\
\hline
\end{tabular}

The cadmium solution was created by dissolving the cadmium source into the solvent and adding the capping ligand in a round bottom flask. The solution was then heated until clear under an argon gas flow of 5 cubic feet per minute at the reaction temperature, $180^{\circ} \mathrm{C}$. The temperature was monitored using a standard glass thermometer. The selenium solution was created by adding elemental selenium to the solvent and TOP under an argon gas flow of 5 cubic feet per minute and stirring in a sonication bath. The concentrations of each material in each solution are shown in Tables 7 and 8.

The selenium solution was then injected into the cadmium solution which was being stirred and heated under an argon flow. The selenium solution added was equal to $10 \%$ of the cadmium solution by volume. The solution was allowed to react for up to four hours at the reaction temperature. The solution was then purified using a 2-propanol(Alfa Aesar, 99\%)/methanol(Alfa Aesar, 99.9\%) mixture to remove any excess reactants. The resulting product was analyzed using XRD, UV-visible Spectroscopy, and Photoluminescence Spectroscopy. 


\subsection{Results and Discussion}

\subsubsection{Co-precipitation Quantum Dot Synthesis Method}

The co-precipitation method as executed resulted in a black solid substance. This substance was analyzed using XRD. The XRD results of the unpurified sample are

displayed in Figure 3. The figure shows that there are clear peaks at $24^{\circ}$ and $30^{\circ}$ with wide or partial peaks at $26^{\circ}$ and $39^{\circ}$. If compared to the XRD analysis of the materials shown in Figure 4 and 5, the $24^{\circ}$ and $30^{\circ}$ peak can be attributed to residual amounts of selenium and the $39^{\circ}$ peak potentially to the cadmium chloride. To extract these materials, a washing procedure was developed and outlined in the experimental section. Since sodium borohydride was used to dissolve the selenium for preparation of the experiment, it was hypothesized that it would only dissolve the selenium and selenium oxide in the product of the experiment. The resultant substance was washed with ethanol to remove the cadmium. After following this procedure, the sample was re-analyzed using XRD, shown in Figure 6 to yield the removal of the two undesirable substances. The peaks from the final product were of cadmium selenide of the $25.7^{\circ}$ and a peak starting to form at $42^{\circ}$ when compared to the peaks previously reported[21]. 


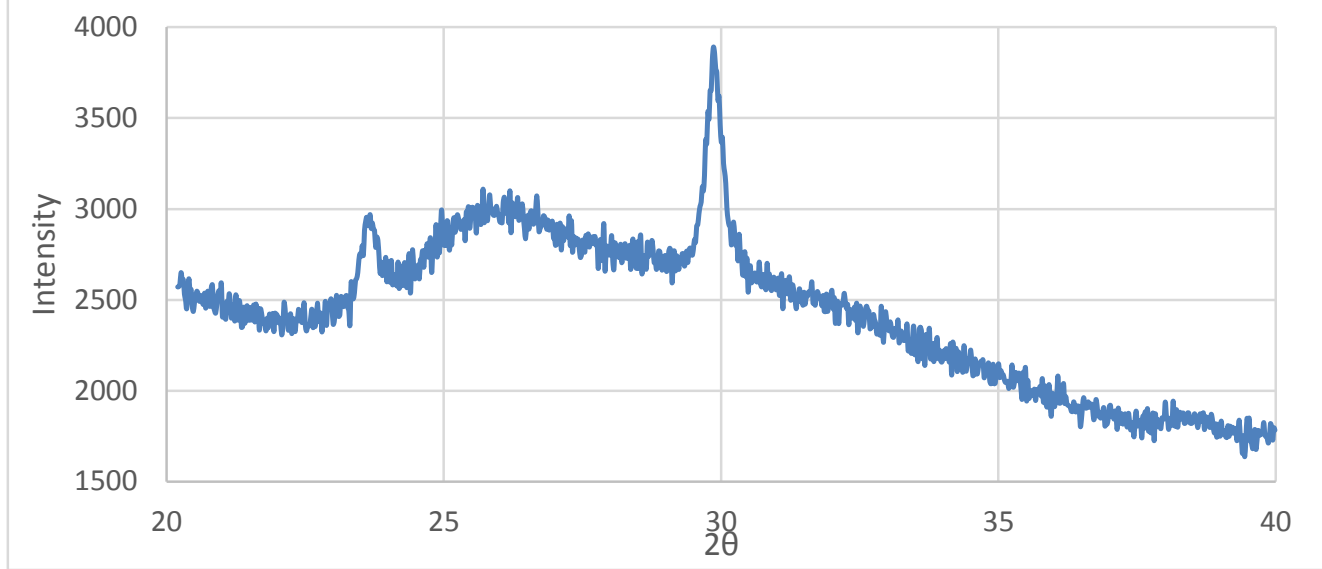

Figure 3: XRD Analysis of Co-precipitation Quantum Dot Synthesis before purification

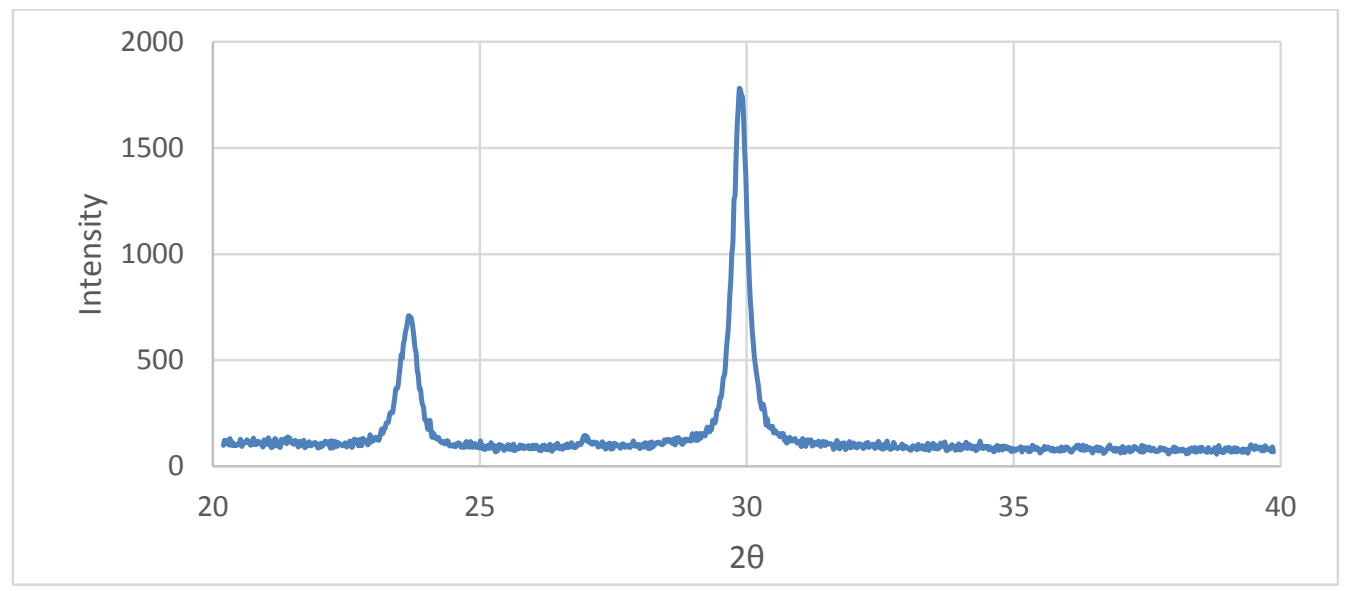

Figure 4: XRD Analysis of the Elemental Selenium

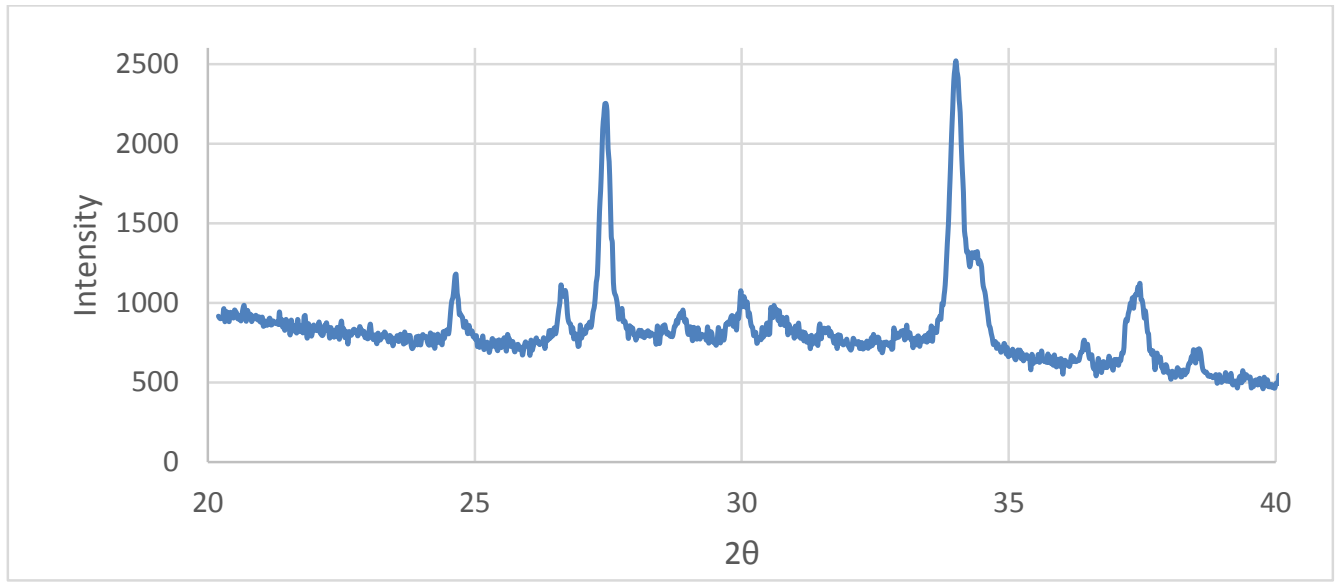

Figure 5: XRD Analysis of Cadmium Chloride 


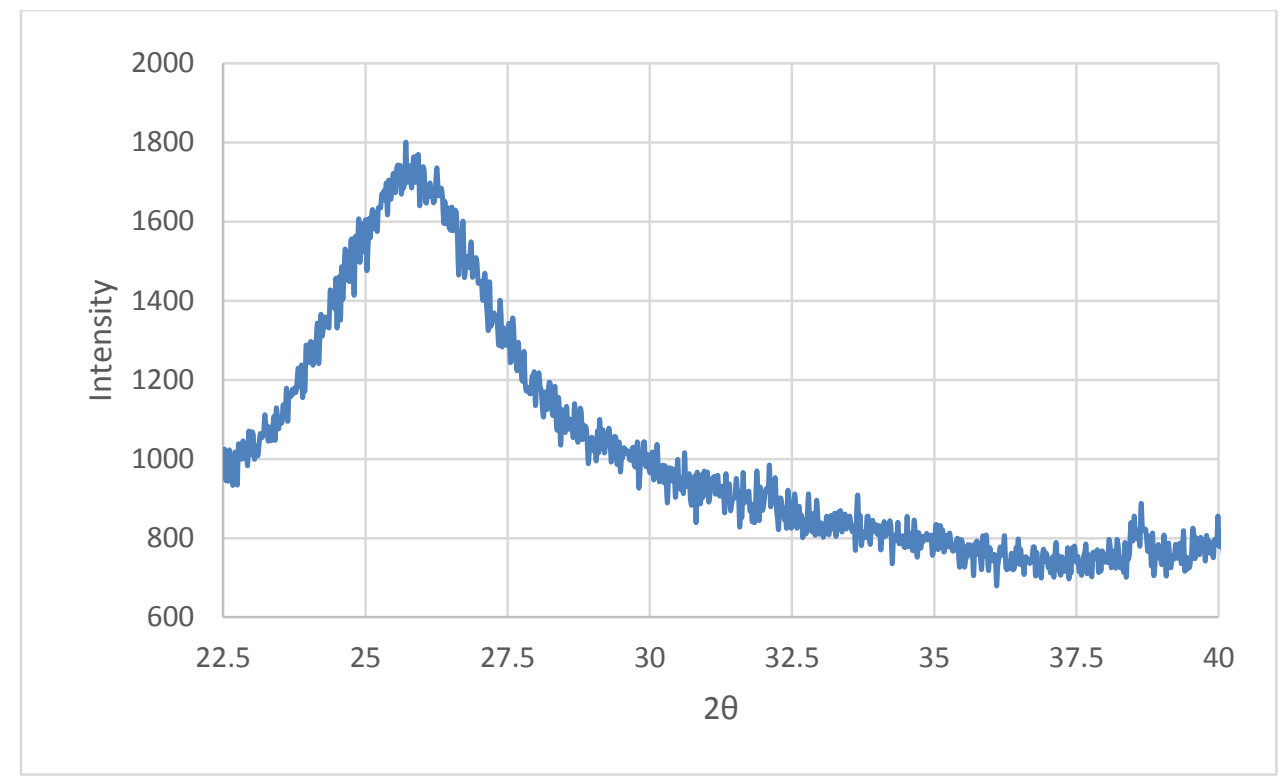

Figure 6: XRD Analysis of Purified CdSe Sample

The cadmium selenide that was formed did not exhibit any fluorescent properties whatsoever. A particle size analysis was completed but yielded $50 \mu \mathrm{m}$ mean effective diameter using the Brookhaven 90Plus Particle Sizer. A TEM image was taken of the sample synthesized by the co-precipitation technique and is displayed in Figure 7. The TEM Analysis shows that the particles have agglomerated into $\sim 1 \mu \mathrm{m}$ particles. Although the light-scattering particle analyzer is only reliable in the range from $2 \mathrm{~nm}$ to $3 \mu \mathrm{m}$, this analysis proves that the method as executed will not yield a fluorescent material for application in a light emitting diode. 


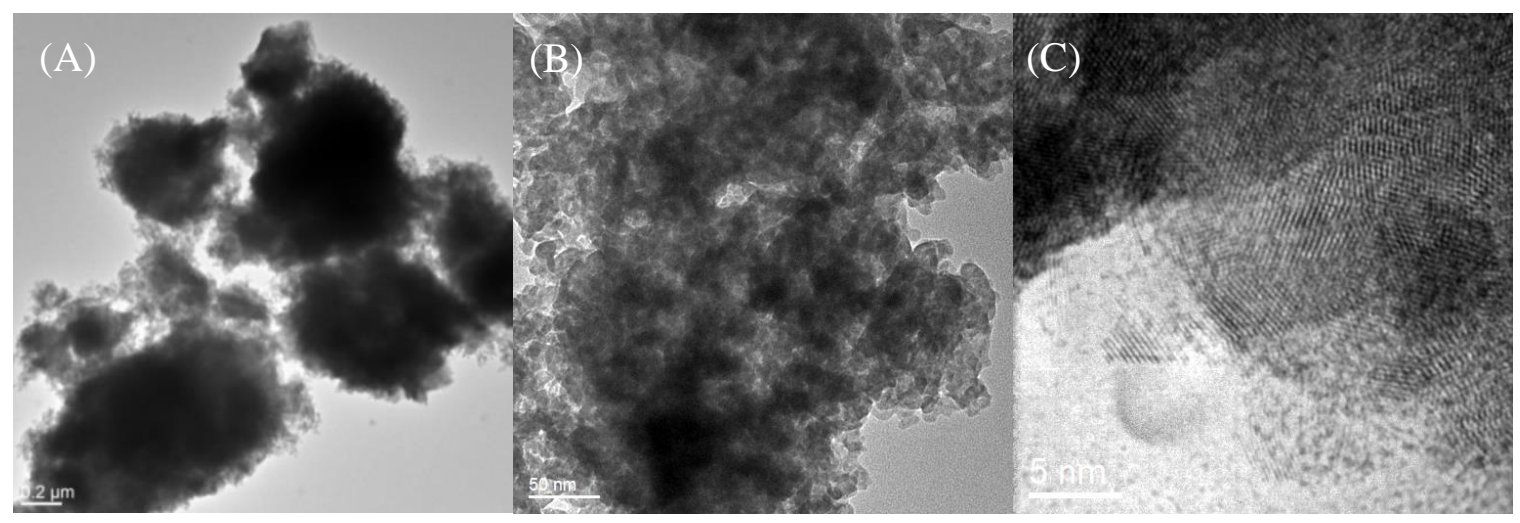

\section{Figure 7: TEM Images of Co-precipitation Quantum Dot Synthesis with Scale Bars of (A) $0.2 \mu \mathrm{m}$, (B) $50 \mathrm{~nm}$ (C) $5 \mathrm{~nm}$}

The main hypothesis for why this method is not sufficient to synthesize nanocrystals is because it lacks a crystal growth inhibitor. As in many nanocrystal syntheses, a small chained polar organic molecule is used to stabilize the highly reactive nanocrystals and impede their growth. As can be deduced from the methodology, this procedure was lacking such a component. Therefore, the growth of the nanocrystals continues until the cadmium selenide can stabilize itself. Since the product of this reaction was unsuccessful, other methods were pursued and detailed in the following sections.

\subsubsection{Reverse Micellar Quantum Dot Synthesis}

The synthesis of quantum dots using reverse micelles involved the creation of a reverse micellar solution. After the solution was created, composition displayed in Table 3, the reverse micelle size was analyzed to ensure an appropriate size for quantum dot synthesis. This analysis was completed using the Brookhaven 90Plus Particle Sizer and yielded an effective diameter of $\sim 30 \mathrm{~nm}$. The polydispersity and baseline index are 0.217 and $0.1 / 14.03 \%$, respectively. Polydispersity is the measure of the non-uniformity's that 
exist in the particle size distribution. The baseline index is the measure of how much the autocorrelation function, which generates the information for the particle size and polydispersity, was distorted by large and rare particles. The baseline index for this sample indicates that this is of bad quality. Since the samples were filtered before being analyzed, the baseline index was attributed to the sample's weak light scattering characteristics which can be attributed to the multiple phases of the reverse micelle. The low average count rate of the analysis would also affect the baseline index in a negative manner. Since the run distribution was narrow and the results were repeatable, it can be assumed the analysis was accurate for the purposes of this study.

The concept behind this method was to essentially create nanosized reaction vessels for the cadmium and selenium using the reverse micelles. An increase in concentration from the co-precipitation method was justified by the scale of the proposed reactor, presumably the volume of multiple reverse micelles. Unlike conventional methods, the reverse micelle inhibits the growth of the nanocrystals by limiting the amount of reactants. Through refluxing of the solution, the micelles were combined, facilitating the reaction.

The hypothesized outcome was the surfactant bonding to the surface of the nanocrystals and creating a stable colloidal suspension of the nanocrystals in the organic phase. While heating the reaction, the aqueous phase of the solution would concentrate faster than the organic phase because of the differences in boiling points. As the refluxing would disrupt the reverse micelles through vaporizing the water inside the micelle, the polar heads of the surfactant would be attracted to the surface of the CdSe because of the dangling ionic bonds on the surface of the CdSe nanocrystals. The result would be an 
alternate method of creating colloidal nanocrystal solutions at lower temperatures with more control over size by manipulating the reverse micelle size.

When the reaction was carried out, the resultant solution was a light red, transparent solution. The solution's absorption of the sample was analyzed using UV Visible Spectroscopy. The normalized spectrum of the reverse micellar solution and the reacted solution are in Figure 8. When these two spectrums are compared, it is clear that they exhibit the same peak at $520 \mathrm{~nm}$ and valleys at $510 \mathrm{~nm}, 540 \mathrm{~nm}$ and $860 \mathrm{~nm}$. The absorbance of the product at these peaks has increased but no blue shift has occurred suggesting that no quantum confinement effects have resulted and nanostructures were not formed. When the sample was dried, the powder sample was analyzed using XRD and the results are displayed in Figure 9. The analysis does not exhibit any peaks characteristic to CdSe but does show peaks $34.4^{\circ}$ and $37.9^{\circ}$ which could be attributed to precursor materials, specifically cadmium chloride.

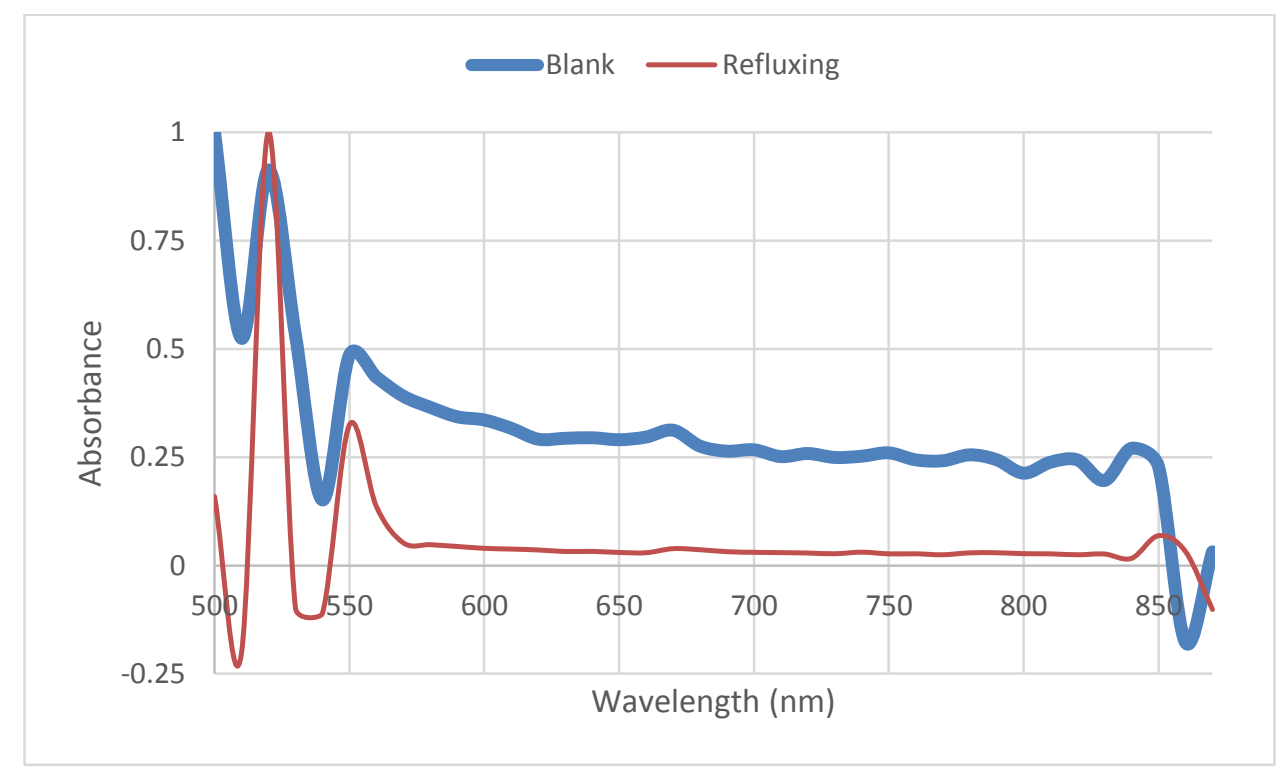

Figure 8: UV-visible Spectroscopy of Blank Micellar Solution and Reacted Micellar Solution 


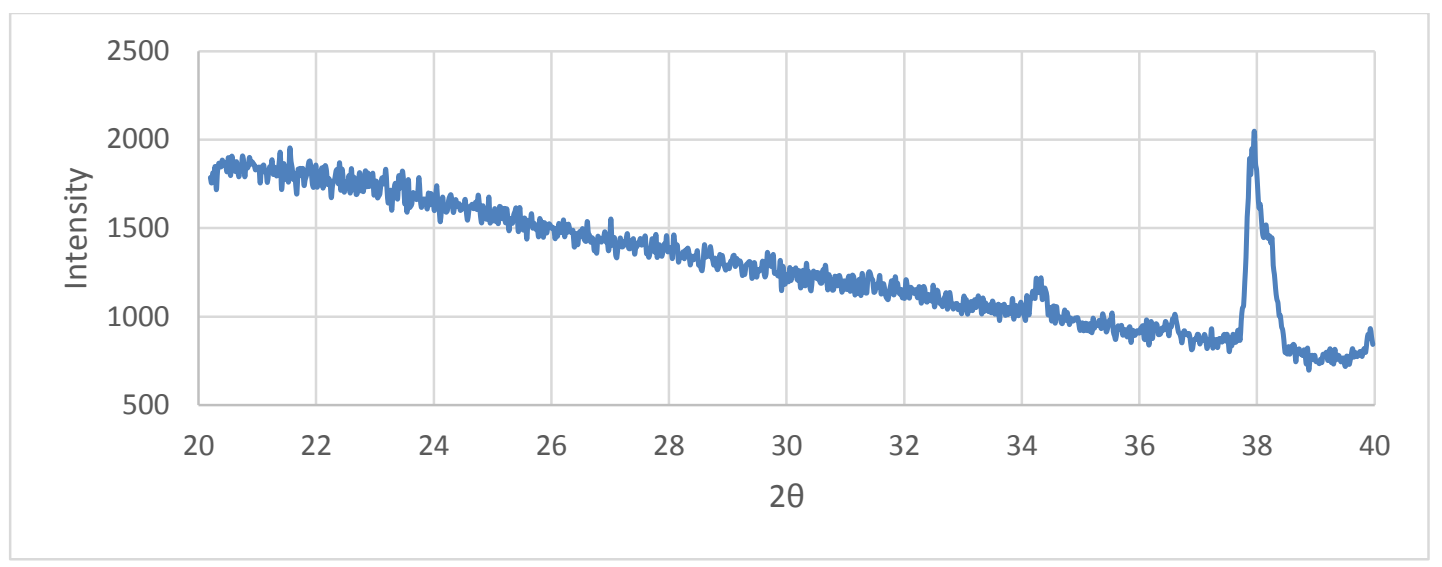

Figure 9: XRD Analysis of the Dried Synthesis Product

These rods were mostly amorphous resulting in the XRD pattern above and making it difficult to draw conclusions on these results alone. TEM images were also taken to help understand the reverse micellar synthesis method. In Figure 10, it can be seen that rods were formed in the synthesis. One important aspect to mention is that reverse micelles seem to form $\sim 50 \mathrm{~nm}$ diameter rods. This might be a result of the reverse micelle solution forming rods instead of sphere which was originally thought. The amorphous nature of the nanorods could be an effect of the operating conditions, such as temperature, concentration of reactants in a reacting reverse micelle and amount of surfactant on the surface of the CdSe structure.

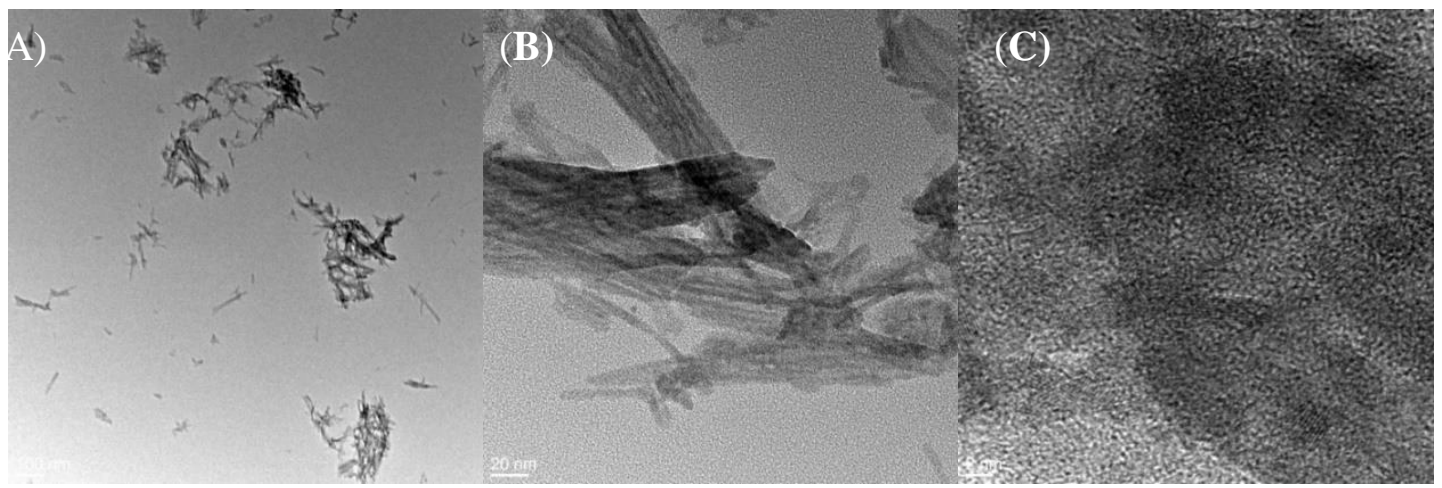

Figure 10: TEM Images of the Reverse Micellar Synthesis Method with scale bars of (A) $100 \mathrm{~nm}$ (B) $20 \mathrm{~nm}$ (C) $2 \mathrm{~nm}$ 
The reverse micellar quantum dot synthesis method is possibly a viable route for quantum dot and quantum rod synthesis. However, it would involve a significant amount of optimization of the reverse micelle size/shape, and concentration of reactants to obtain a high quality quantum dot or rod. Furthermore, using the surfactant as the potential capping agent may not adequately stabilize the growth of the quantum dots. Using a capping agent to stabilize the reverse micelles, such as a mercaptan acid, instead of the 1hexanol may be better at accomplishing the goal of stabilizing the quantum dot. As long as the ligand used stays below a chain length of 10 carbons, the ligand can replace the alcohol and retain its stabilizing affects. [22, 23]

\subsubsection{Hot Injection Method of Quantum Dot Synthesis}

Cadmium selenide quantum dots were synthesized using the hot injection method outlined in the experimental section of Chapter 2. The samples were analyzed using Photoluminescence Spectroscopy and XRD.

The analysis of an oleic acid/TOP/CdSe in 1-octadecene is summarized in Table 9 with the PL spectrum, XRD, and an image of the sample under UV illumination in Figures 11 through 13 . The concentration of the cadmium solution was $2 \mathrm{mM}$ and the concentration of the selenium solution was $20 \mathrm{mM}$. The combination has oleic acid (5.3\% by volume) and TOP ( $1 \%$ by volume) as the capping agents for the nanocrystals. The radiated wavelength was in the yellowish green region of the visible spectrum with a peak wavelength of $560 \mathrm{~nm}$ and a Full Width Half Maximum (FWHM) of $119 \mathrm{~nm}$. The FWHM illustrates a large distribution in the size of the quantum dots. The radiated wavelength can be visually observed through exciting the sample with a UV light as 
shown in Figure 13. UV-Vis Spectroscopy was inconclusive due to the magnitude of the absorbance spectrum of cuvette.

Table 9: Luminescent Properties Summary of Oleic Acid/TOP capped CdSe

\begin{tabular}{|l|l|}
\hline Excitation Wavelength & $442 \mathrm{~nm}$ \\
\hline Peak Wavelength & $560 \mathrm{~nm}$ \\
\hline Full Width Half Max & $119 \mathrm{~nm}$ \\
\hline
\end{tabular}

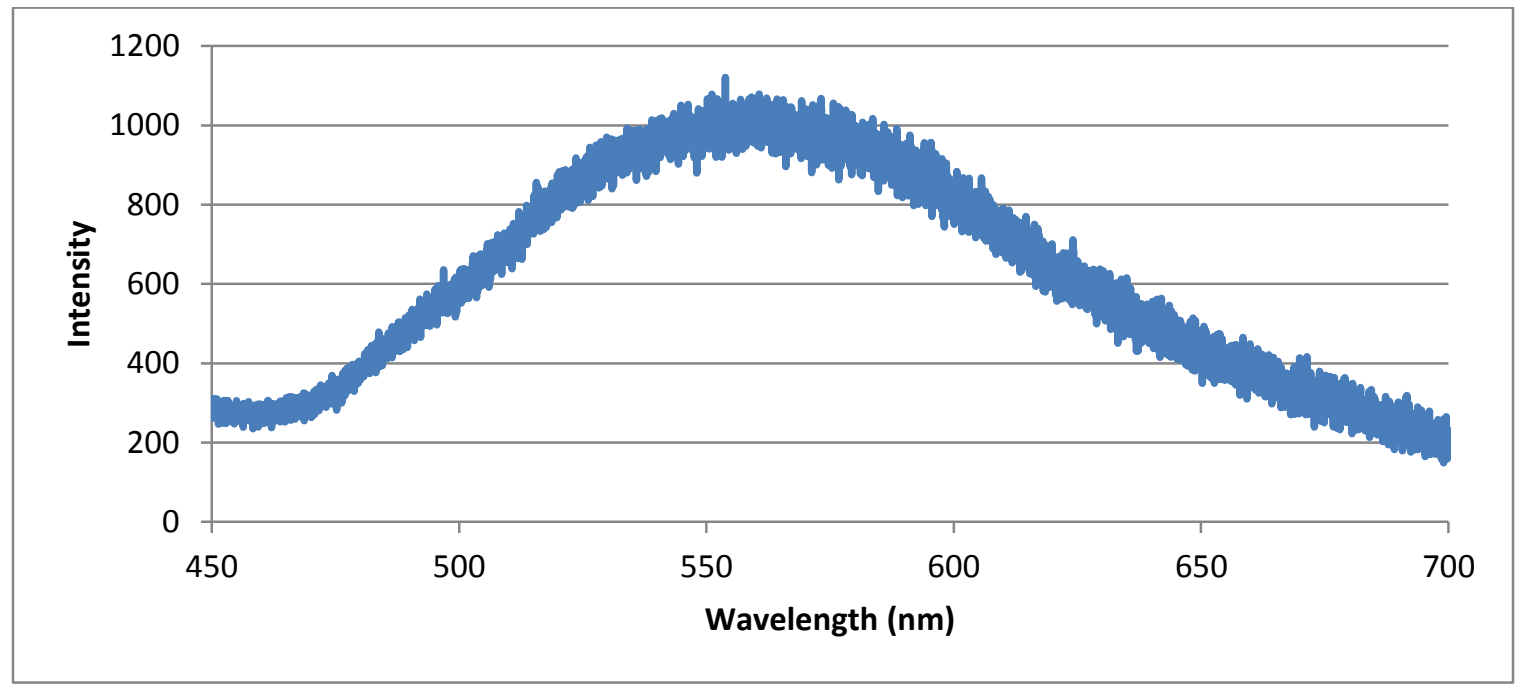

Figure 11: Photoluminescence Spectroscopy of Oleic Acid/TOP capped CdSe 


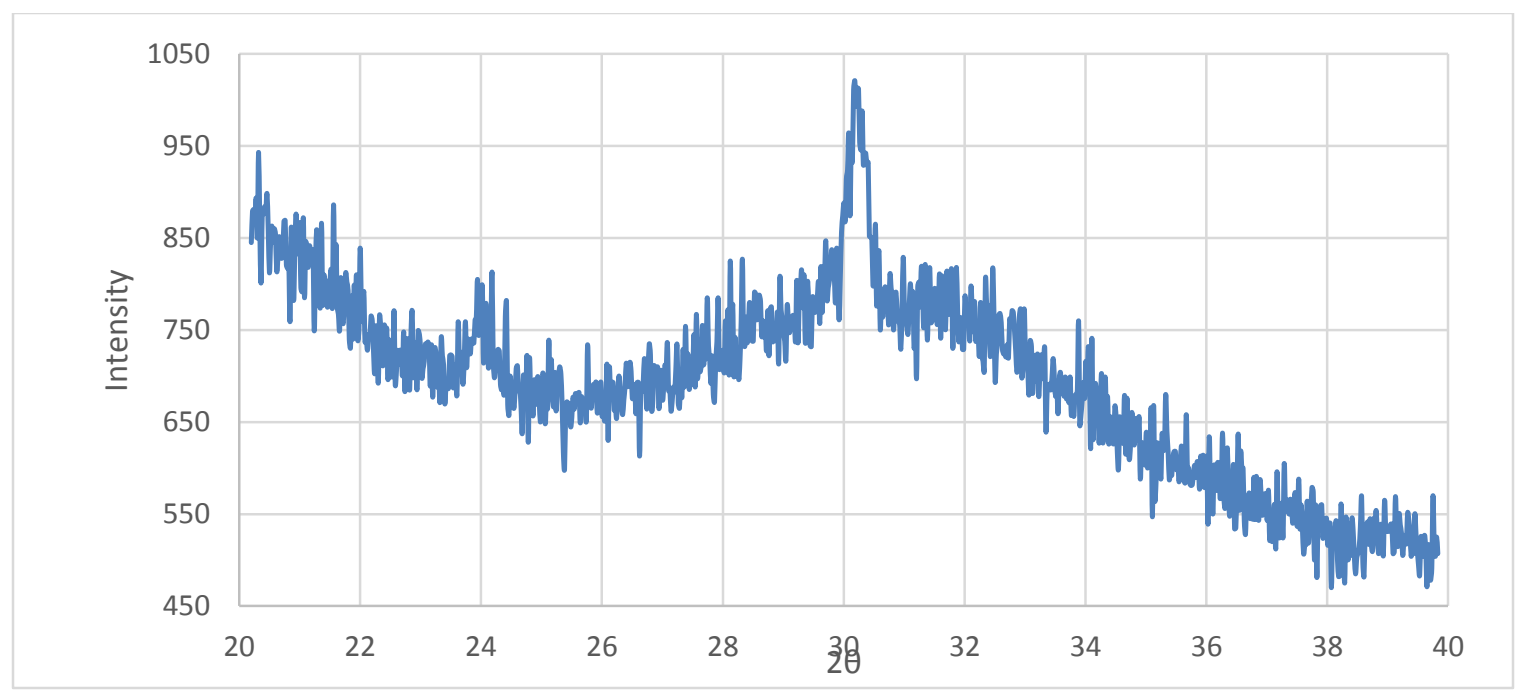

Figure 12: XRD of Oleic Acid/TOP capped CdSe

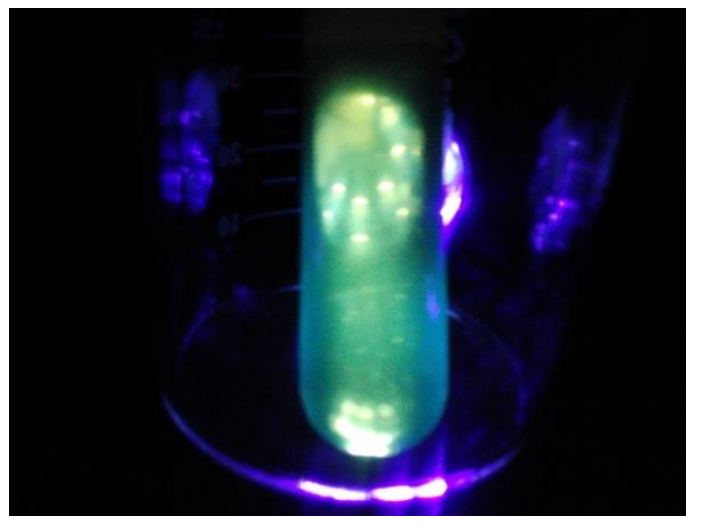

\section{Figure 13: Image of Oleic Acid/TOP capped CdSe under UV Torch}

The method of using oleic acid as the capping agent has been used before in other systems with different solvents to produce highly luminescence materials.[4, 24-26] The use of 1-octadecene/oleic acid/TOP system had not been used up to this point. It was found that this system produced a highly stable colloidal suspension. This was due to the molecular similarities between oleic acid and 1-octadecene. The suspension has been able to keep its fluorescence properties for $>6$ months without flocculating out of solution. The process was also successively scaled up from a volume of $22 \mathrm{~mL}$ to a volume of 110 
$\mathrm{mL}$ without any degradation of luminescence properties or change in characteristic wavelength.

Through increasing the concentration of the cadmium and selenium, it is shown that the size can be altered. When increasing the concentration by two orders of magnitudes to $0.2 \mathrm{M}$ and $2 \mathrm{M}$ of the cadmium and selenium solutions, respectively, the results were not favorable. The reaction was carried out too quickly and the ligand was not able to inhibit the growth of the crystal. When the concentration was only increased by one order of magnitude, PL spectrum was formed and is displayed in Figure 14 and summarized in Table 10. The resultant peak wavelength of this combination exhibits a red shift to $604 \mathrm{~nm}$ and a FWHM of $57 \mathrm{~nm}$. This suggests that the size of the quantum dots has increased from the lower concentration but have developed a smaller size distribution. Since the reaction is carried out at a lower temperature, the crystallinity of the nanocrystals must not be of an acceptable quality to promote a high quantum yield. The lack of acceptable crystallinity would promote charge traps and lead to non-radiative recombinations of excitons.

Table 10: Luminescent Properties of Oleic Acid/TOP capped CdSe with increased concentration by one order of magnitude

\begin{tabular}{|l|l|}
\hline Excitation Wavelength & $442 \mathrm{~nm}$ \\
\hline Peak Wavelength & $604 \mathrm{~nm}$ \\
\hline Full Width Half Max & $57 \mathrm{~nm}$ \\
\hline
\end{tabular}




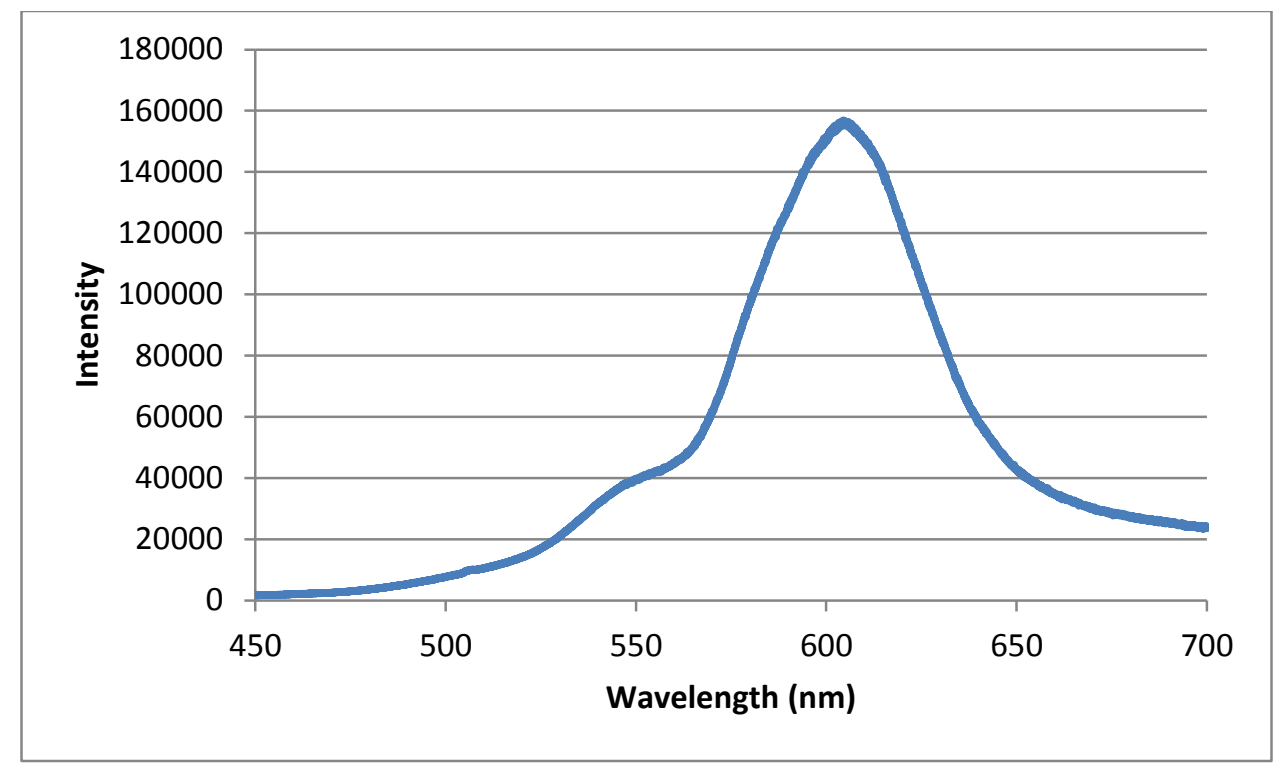

Figure 14: Photoluminescence Spectroscopy of Oleic Acid/TOP capped CdSe with increased concentration by one order of magnitude

An attempt to create a directly printable or nearly directly printable solution during quantum dot synthesis. In attempts to create an inkjet solution, cyclohexanol was used as a viscosifying agent to get the solution within a certain range of viscosity. Therefore, there was an attempt to use cyclohexanol as the solvent. During these brief trials, only the solvent was changed and oleic acid/TOP was used as the capping agent. Unfortunately, the cyclohexanol had a negative effect on the capping agent's ability to inhibit the growth of nanocrystal.

The analysis of mercapto-benzoic acid/TOP capped CdSe QD is summarized in Table 11 with the PL spectrum and XRD of the sample in Figures 15 and 16. The combination involves mercapto-benzoic acid at a concentration of $2 \mathrm{mM}$ and TOP at a concentration of $1 \%$ by volume. The radiated wavelength was in the green region of the visible spectrum with a wavelength of $510 \mathrm{~nm}$ and a FWHM of $111 \mathrm{~nm}$. The FWHM illustrates a large distribution in the size of the quantum dots. Mercaptans (-SH) are among the most used ligands when creating quantum dots. [4] This is due to their ability 
to passivate the surface of the quantum dot. It is clear that under the same conditions as the two other ligands used in this study, the emissive peak has a smaller wavelength which corresponds to a smaller quantum dot size. Meaning, the mercapto-benzoic acid was able to inhibit the growth of the quantum dot more effectively than the other two ligands. This reaction was also easily scaled up from $22 \mathrm{~mL}$ to $110 \mathrm{~mL}$ without any degradation of luminescence properties or change in characteristic wavelength. The XRD pattern does not show any peaks that would be associated with a CdSe Quantum dot. This may be due to the excessive amount of ligand passivation on the surface. It may also be due to an amorphous CdSe phase on the surface of the quantum dot itself. An attempt was made to crystalize the amorphous layer by heating but resulted in irreversible agglomeration of the crystals. A larger range of angles in the XRD analysis may show peaks or a slower scan may reveal peaks that cannot be seen due to noise.

Table 11: Luminescent Properties Summary of mercapto-benzoic acid/TOP capped CdSe QD

\begin{tabular}{|l|l|}
\hline Excitation Wavelength & $442 \mathrm{~nm}$ \\
\hline Peak Wavelength & $510 \mathrm{~nm}$ \\
\hline Full Width Half Max & $111 \mathrm{~nm}$ \\
\hline
\end{tabular}




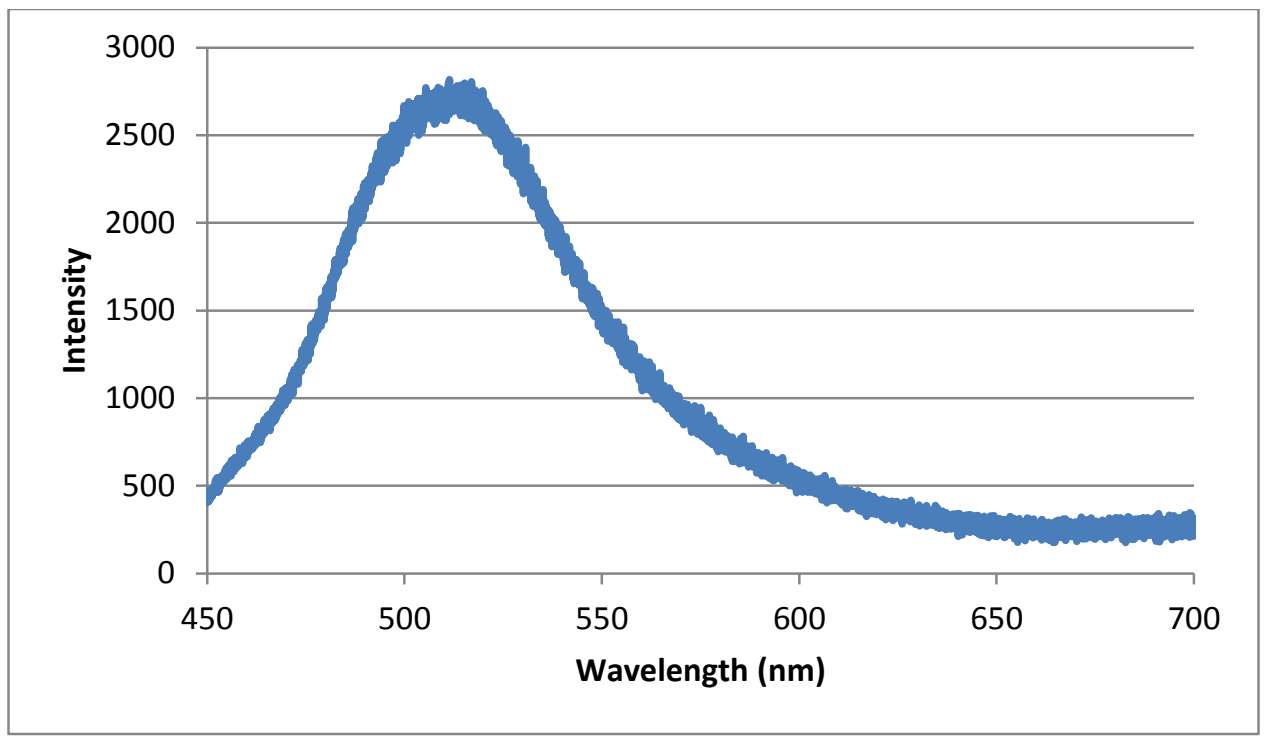

Figure 15: PL Spectroscopy of mercapto-benzoic acid/TOP capped CdSe QD

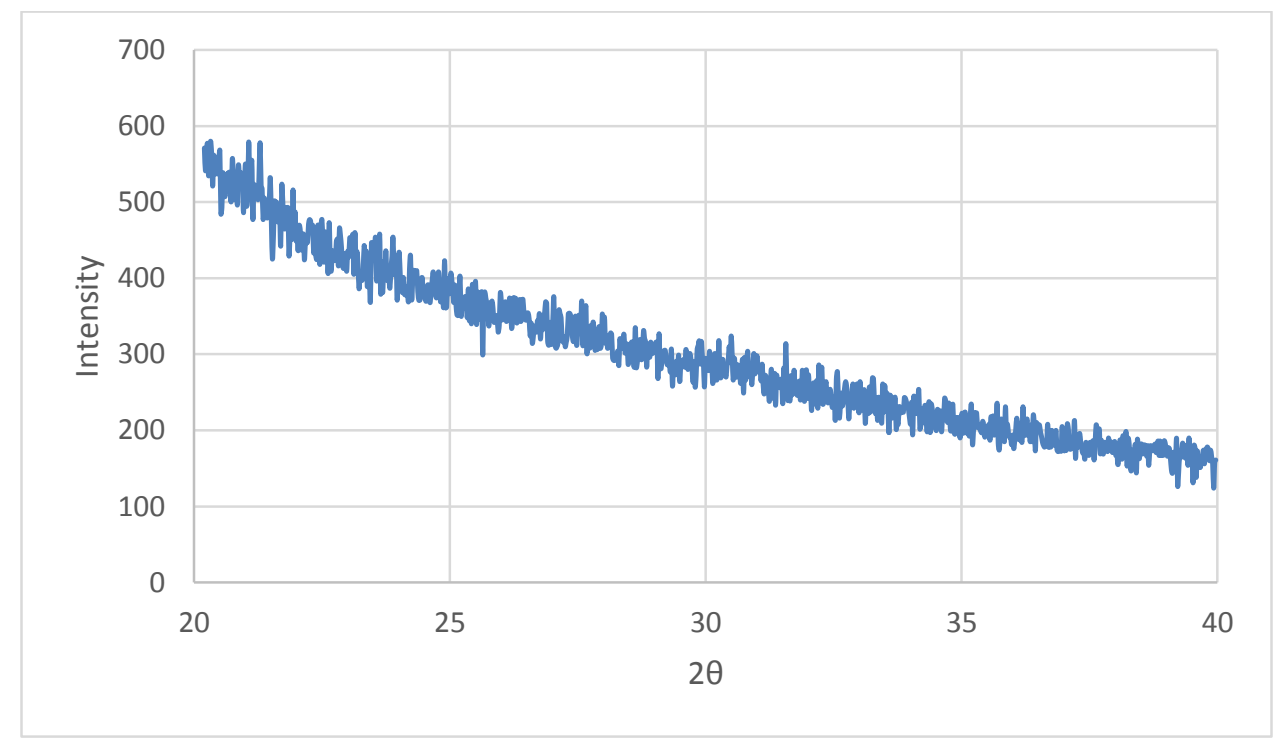

Figure 16: XRD of mercapto-benzoic acid/TOP capped CdSe QD

The analysis of a benzene-1,4-dithiol/TOP capped CdSe QD is summarized in Table 12 with the PL spectrum and XRD of the sample in Figures 17 and 18. Benzene1,4-dithiol was used at a concentration of $2 \mathrm{mM}$ and TOP at a concentration of $1 \%$ by volume. The radiated wavelength was in the green region of the visible spectrum with a wavelength of $555 \mathrm{~nm}$ and a FWHM of $110 \mathrm{~nm}$. The FWHM illustrates a large distribution in the size of the quantum dots. The use of this ligand is analogous with the 
use of the mercapto-benzoic acid. As can be seen, the lack of acid functional group leads to a lesser ability to passivate the surface, although still resulting in a quantum dot. The XRD pattern for this product does show a peak characteristic to a CdSe quantum dot with a peak around $20^{\circ}$. This ligand does have a slightly shorter chain length and therefore my not inhibit the x-ray's from diffracting.

Table 12: Luminescent Properties Summary of benzene-1,4-dithiol/TOP capped CdSe QD

\begin{tabular}{|l|l|}
\hline Excitation Wavelength & $442 \mathrm{~nm}$ \\
\hline Peak Wavelength & $555 \mathrm{~nm}$ \\
\hline Full Width Half Max & $110 \mathrm{~nm}$ \\
\hline
\end{tabular}

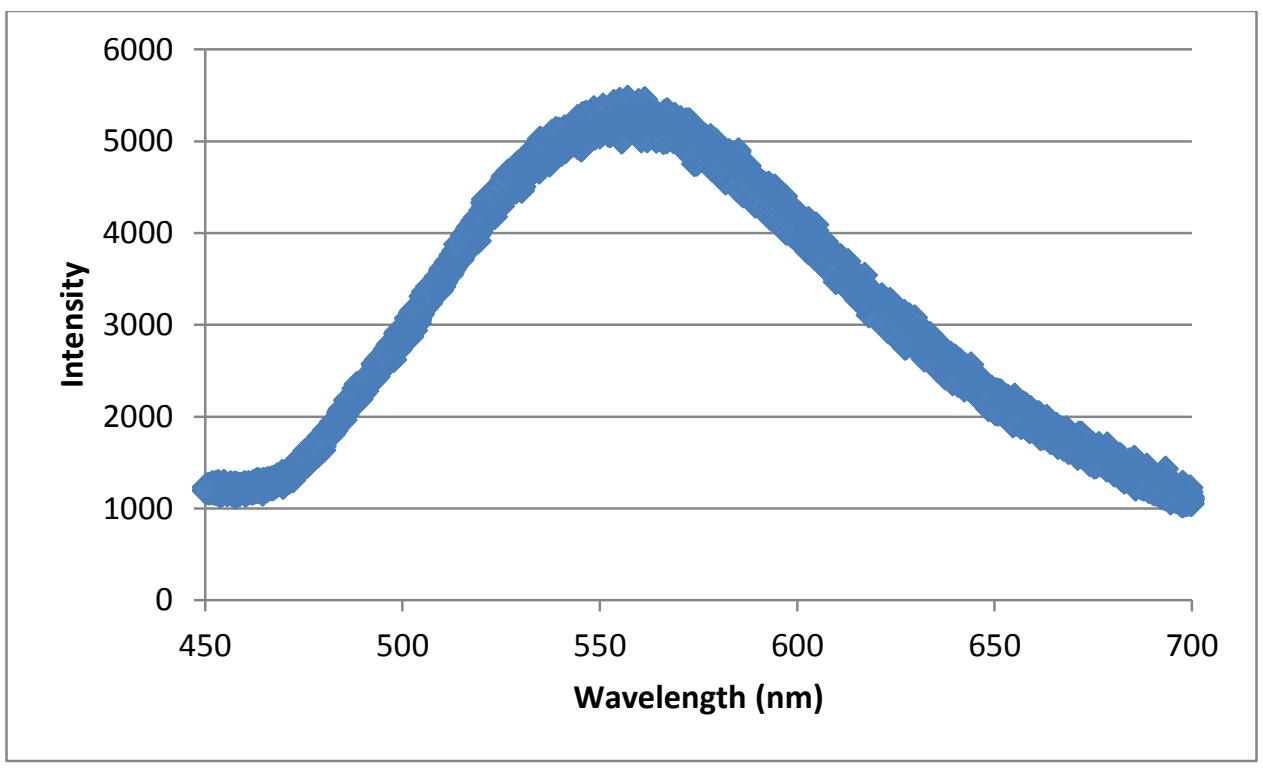

Figure 17: Photoluminescence Spectroscopy of benzene-1,4-dithiol/TOP capped CdSe QD 


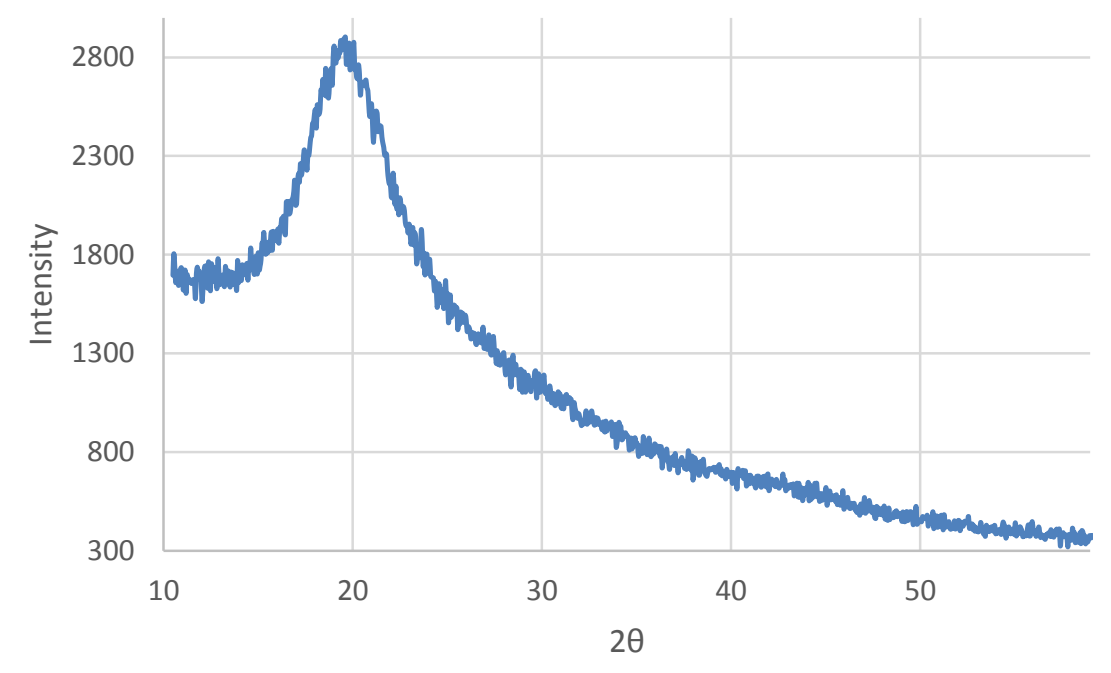

Figure 18: XRD of benzene-1,4-dithiol/TOP capped CdSe QD

Quantum dots were also purchased from NN-Labs which specializes in the synthesis of various types of quantum dots. The quantum dots which were purchased were core-shell CdSe-ZnS. Their characteristic wavelength was $560 \mathrm{~nm}$ with a FWHM of $40 \mathrm{~nm}$ as displayed in Table 15 and in Figure 19. They came dispersed in toluene and were stated to be stable for up to one year. These quantum dots were of obvious better quality than the sample produced by the previously mentioned procedures. This was mostly due to the $\mathrm{ZnS}$ shell layer which significantly enhances the quantum yield. These quantum dots are a prime example of the importance of an inorganic passivation layer to reduce dangling bonds and charge traps. While developing the device and synthesis methods, the NN-Labs quantum dots were mainly used as the emissive layer of the device to ensure performance of the device. 
Table 13: Luminescent Properties of NN-Labs Quantum Dots

\begin{tabular}{|l|l|}
\hline Excitation Wavelength & $442 \mathrm{~nm}$ \\
\hline Peak Wavelength & $560 \mathrm{~nm}$ \\
\hline Full Width Half Max & $40 \mathrm{~nm}$ \\
\hline
\end{tabular}

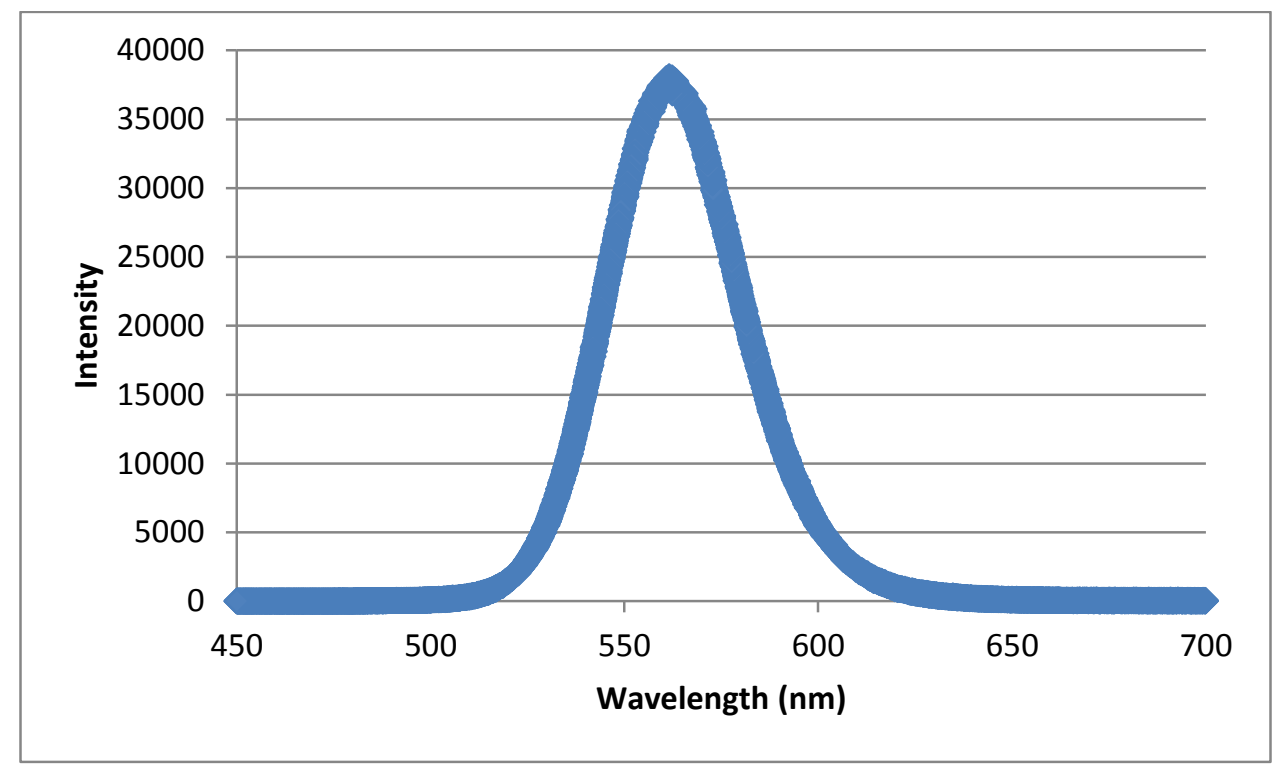

Figure 19: Photoluminescence Spectroscopy of Quantum Dots purchased from NNLabs 


\section{DEVELOPMENT OF QUANTUM DOT LIGHT EMITTING DIODES}

\subsection{Introduction}

The key to fabricating an efficient light emitting diode is creating defect-free layers which promote radiative relaxation of electrons. This is done with uniform, continuous layers of material which are strategically arranged to promote light emission. The strategy of organizing materials is known as band gap engineering. This technique attempts to use the work functions and energy band gaps of different materials to guide electrons and holes to the conduction and valence bands of an emissive layer, respectively. By doing so, excitons should form and relax in the emissive layer and create photons. Although one layer can be used to produce light, other layers such as electron injection layer (EIL), electron transport layer (ETL), hole blocking layer (HBL) and their counterparts can be used as "stepping stones" from the metal contact to the emissive 
layer's energy band gap. These other layers are also used to reduce shorting of the thin film device and to provide a barrier to the diffusion of the metals from the contacts into the emissive layer. [7] Figure 20 is an energy band gap diagram of a QD-LED designed to trap carriers in the emissive layer.

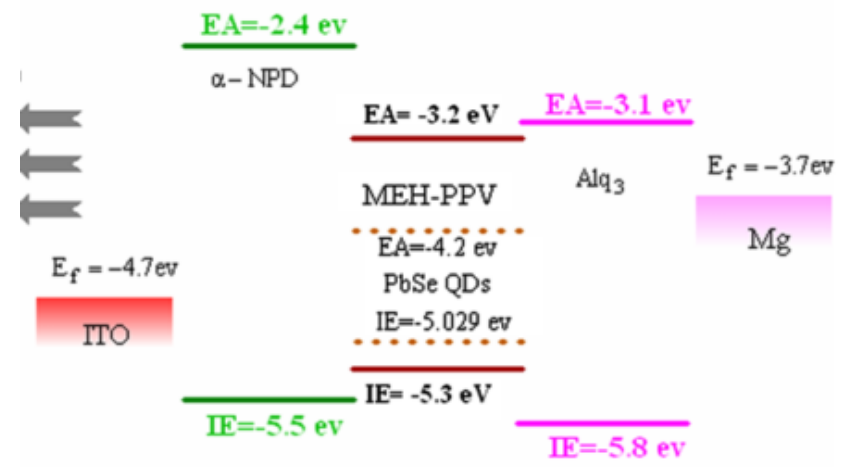

Figure 20: Typical Energy Band Gap Diagram [27]

It is understood that electrons prefer to be the lowest energy state while holes prefer to be in the highest energy state. The $\mathrm{Alq}_{3}$ (electron transport) is used to increase the energy of the electron from the $\mathrm{Mg}$ (cathode) so as to decrease in energy to the PbSe QDs (emissive layer) while the NPD (hole transport) is used to decrease the energy of the hole so as to promote transfer to the $\mathrm{PbSe}$ QDs where the electron combines with the hole or relaxes to form a photon. Many additional layers can be deposited to ensure the appropriate charge transfer.

The initially proposed structure of the device which is displayed in Figure 21 (A) is ITO/PEDOT/CdSe QD/Au. The energy band gap structure is displayed in Figure 21 (B). The holes would approach from the ITO and PEDOT. The electrons approach from the gold where excitons form in the quantum dot layer to produce photons. 

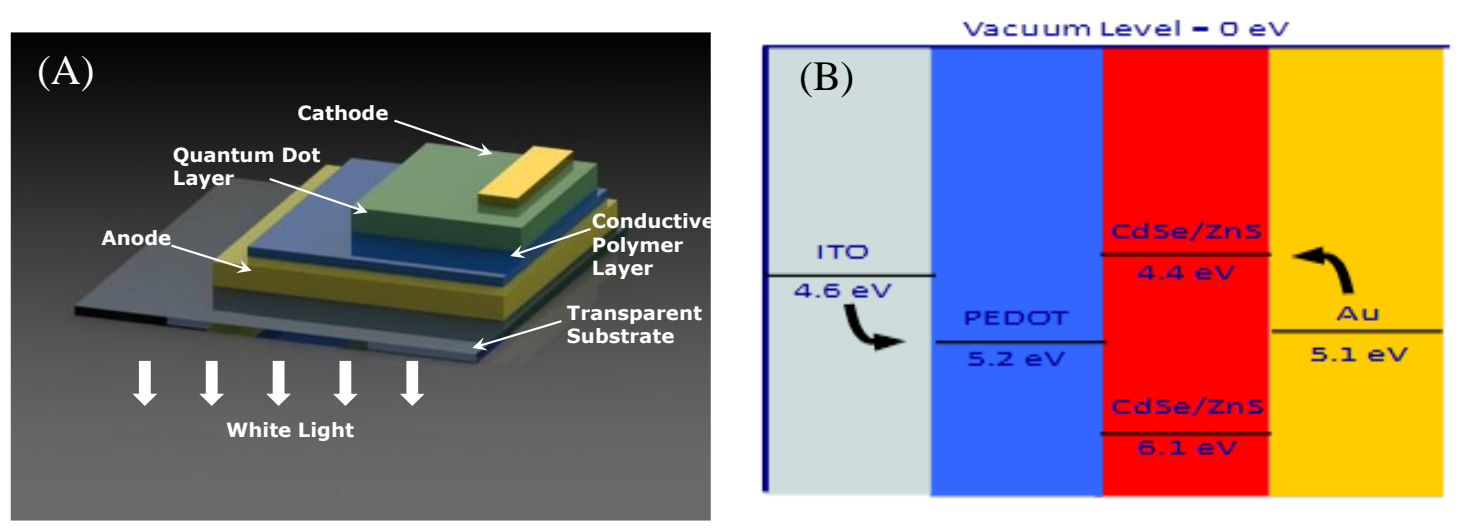

\section{Figure 21: Proposed Device Structure of Hybrid LED; (A) 3-D General Depiction of Device; (B) Proposed Materials and Energy Band Gap Diagram of Device}

The reason for this design was to be able to inkjet print the entire device, at least the polymer and quantum dot layers. This leads to why the ability to synthesis and manipulate the quantum dots in house would be so important. Many of the quantum dots, including the ones purchased from NN-Labs, come in small volumes of dilute solution at high prices. While trying to concentrate these solutions, the entire product could be ruined. Thus, the ability to synthesis quantum dots is the ability to produce concentrated solution for use in inkjet printing.

One important aspect of these thin film devices is layer thickness. The thickness of the layers will determine how well a layer will confine the excitons in the emissive layer. For the PEDOT layer, a water-soluble PEDOT:PSS composite was used as it can be purchased in a solution and readily inkjet printed. [28] The optimal thickness when using PEDOT:PSS as a hole injection layer was found to be between 40-60 $\mathrm{nm}$ when used in polymer light emitting diodes. [29, 30] Based on prior reports, the quantum dot layer produces the most efficient device when a monolayer is formed. [31] A continuous layer is needed and a multiple layers will produce a working device but multiple layers will introduce charge traps and decrease efficiency. 
One of the most effective ways of producing a monolayer is through phase separation of a polymer/quantum dot solution during deposition of the layer. [32] There are many ways that this layer can be deposited, including spin coating and more importantly, inkjet printing. The QDs and the polymer need to form a homogeneous solution. As the solvent evaporates during deposition, the polymer and the QD need to separate into two layers. The ability to do this depends on the solubility of the nanoparticles in the polymer and how to promote the separation of the phases through varying temperature, chain length of polymer and type of ligand.

During the initial fabrication of the device, some alteration were needed to help facilitate the production of light. This means that layers were added to the device structure. Table 16 and 17 provide energies for the valence and conduction bands of typical materials used in quantum dot light emitting diodes. [5]

Table 14: Valence and Conduction band energies for some commonly used organics of quantum dot light emitting diodes

\begin{tabular}{|c|c|c|}
\hline Organics & $\begin{array}{c}\text { Conduction } \\
\text { Band }(\mathrm{eV})\end{array}$ & $\begin{array}{c}\text { Valence } \\
\text { Band }(\mathrm{eV})\end{array}$ \\
\hline Alq3 & 3.1 & 5.8 \\
\hline CBP & 2.9 & 6 \\
\hline PBD & 2.6 & 6.1 \\
\hline PCBM & 4 & 6.5 \\
\hline PPV & 2.5 & 5.1 \\
\hline PVK & 2.2 & 5.3 \\
\hline TAZ & 3 & 6.5 \\
\hline TFB & 2.2 & 5.4 \\
\hline TPBI & 2.7 & 6.2 \\
\hline TPD & 2.1 & 5.4 \\
\hline Poly TPD & $2.3 / 2.5$ & $5.2 / 5.4$ \\
\hline
\end{tabular}


Table 15: Work function of commonly used anode and cathode materials in quantum dot light emitting diodes

\begin{tabular}{|c|c|}
\hline Materials & $\begin{array}{c}\text { Work function } \\
(\mathrm{eV})\end{array}$ \\
\hline $\mathrm{Al}$ & 4.1 \\
\hline $\mathrm{Ag}$ & 4.6 \\
\hline $\mathrm{Ca}$ & 2.9 \\
\hline $\mathrm{ITO}$ & 4.6 \\
\hline $\mathrm{LiF} / \mathrm{Al}$ & 2.8 \\
\hline $\mathrm{Mg}$ & 3.7 \\
\hline PEDOT:PSS & 5 \\
\hline
\end{tabular}




\subsection{Experimental}

\subsubsection{Preparation of Conductive Slides}

Two different types of conductive glass slides were used as the substrate and anode for the device. An Indium Tin Oxide (ITO) coated glass slide with resistance of 5$15 \mathrm{ohms}$ per $\mathrm{cm}^{2}$ and dimensions of $25 \mathrm{~mm} \times 25 \mathrm{~mm} \times 1.1 \mathrm{~mm}$ were used and provided by Delta Technologies (part no. CB-50IN-0111). Fluorine doped Tin Oxide (FTO) glass slides were also used as the cathode with a conductivity of 10-20 ohms and dimensions of $25 \mathrm{~mm} \times 50 \mathrm{~mm} \times 2.3 \mathrm{~mm}$ and provided by Hartford Glass Co. Inc.

The slides were prepared for device fabrication by a multi-step cleaning process. The first step involved the cleaning of the slides with dish soap and deionized (DI) water. The slides were then placed in Isopropyl alcohol (IPA) (EMD Millipore, 70\%) in individual falcon tubes, sonicated for five (5) minutes and rinsed with DI water. This step was repeated three (3) times. The glass slides were then placed in a PLAMAX 150S for plasma treatment with argon and oxygen for fifty (50) cycles followed immediately by the deposition of the polymer layer.

\subsubsection{Deposition of Polymer Layer}

There are two ways in which poly(3,4-ethylenedioxythiophene) (PEDOT) composite was deposited as a thin film on the glass slides. One method involves the chemical vapor deposition (CVD) of EDOT, the PEDOT monomer which was completed and provided by Dr. Vaddiraju from the Texas A\&M University. The slides were characterized by an Alpha-Step 500, Surface Profiler, for surface thickness and roughness 
and XE-100 Advanced Scanning Probe Microscope for surface morphology and surface roughness.

The other method of polymer deposition involved spin coating of an aqueous PEDOT-based solution produced from a Poly(3,4-ethylenedioxythiophene)poly(styrenesulfonate) (PEDOT-PSS) $3.0-4.0 \mathrm{wt} \%$ in $\mathrm{H} 2 \mathrm{O}$, high-conductivity grade solution purchased from Sigma-Aldrich (part no. 655201-25G). The spin-coated solution was created by addition of varying amounts of the PEDOT:PSS solution and ethanol (Decon Labs, 200 Proof) by volume to optimize the thickness of the layer. The solution was then sonicated using a Virsonic 100 Sonicating Probe for fifteen (15) minutes.

The polymer solution was then spin coated using an in-house assembled spin coater, shown in Figure 22. The cleaned slides have a piece of scotch tape over roughly seven (7) millimeters of one end of the slide. The scotch tape was used to protect a portion of the ITO from the other layer to create a bottom contact for the device. 200 microliters of the solution was dropped onto the cleaned slides in the spin coater using a micropipette, ensuring that the entire surface was wetted with the solution. The spin coater was then turned to $1000 \mathrm{rpm}$ for 60 seconds. Two layers were placed on each slide before placed in the vacuum oven over night at $80^{\circ} \mathrm{C}$ and $15 \mathrm{~mm} \mathrm{Hg}$ absolute. 


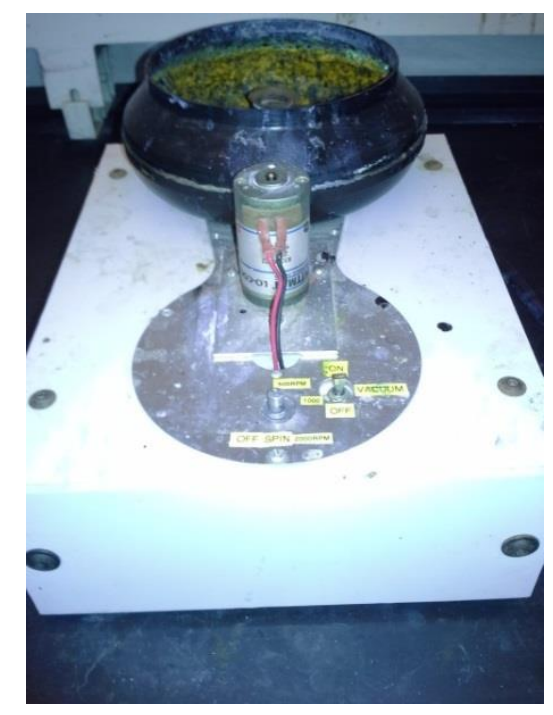

Figure 22: Spin Coater

\subsubsection{Deposition of Quantum Dot Layer via Spin Coating}

The quantum dots were deposited onto the polymer coated slide in various solutions of differing compositions using spin coating. Scotch tape was used to mask the remainder of the device to create a uniform continuous layer strictly on the polymer. When spin coating of the quantum dot solution, $200 \mu \mathrm{L}$ was deposited onto the slide using a micropipette and spun at $1000 \mathrm{rpm}$ for 60 seconds. It is important to note that complete wetting of the surface is important for a continuous, uniform layer. Depending on the quantum dot solution sample, the number of layers would be varied to provide a continuous layer. The slides were then kept in a desiccator for storage until additional layers were deposited.

\subsubsection{Deposition of Quantum Dot Layer via Inkjet Printing}

The inkjet printer used in this experiment was a Dimatix Materials Printer DMP2800 Series, displayed in Figure 23. The printer had a drop watcher camera to optimize 
the waveform and piezoelectric printhead voltages. It also had the ability to heat the print head, ink, and substrate up to $60^{\circ} \mathrm{C}$. Each printed pattern was custom for each sample to optimize the coverage using the printer software.

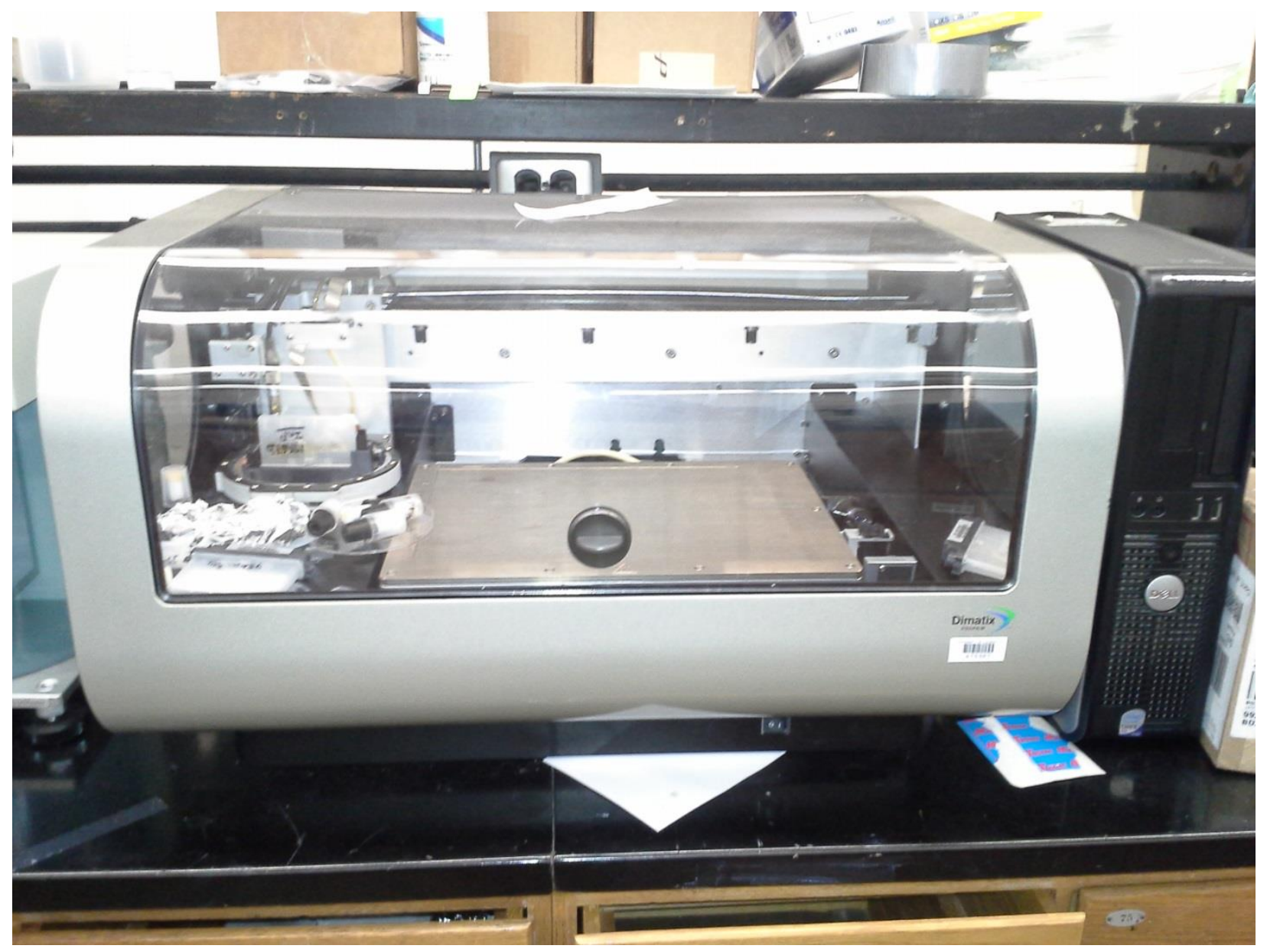

Figure 23: Dimatix Materials Printer DMP-2800 Series

The first step of inkjet printing was developing a printable ink or solution. The main parameters that dictated printability were viscosity, surface tension, and particle size. The latter of these parameters was mitigated by filtering the solution using $0.2 \mu \mathrm{m}$ filters before filling the inkjet cartridge. The viscosity and surface tension were altered by changing the composition of the solution with an optimal range of $10-15 \mathrm{cP}$ for the viscosity and $25-35 \mathrm{mN} / \mathrm{m}$ for surface tension as determined by the printer manufacturer. 
An Anton Paar AMVn Automated Microviscometer was used to test the various samples of quantum dot inks for viscosity. Figure 24 displays the microviscometer used to perform the viscosity studies. To complete the surface tension studies, a DyneMaster Automatic Surface Tensiometer was utilized which is displayed in Figure 25. The fluid dynamics inside an inkjet printhead are highly complex and when dealing with liquids that have the potentially for high amounts of dissolved gases, such as aqueous solutions, a degasing step was required to prevent bubbles from forming in the inkjet printhead. This was completed by placing the solution in a vacuum for one hour at 15 in $\mathrm{Hg}$ absolute pressure.

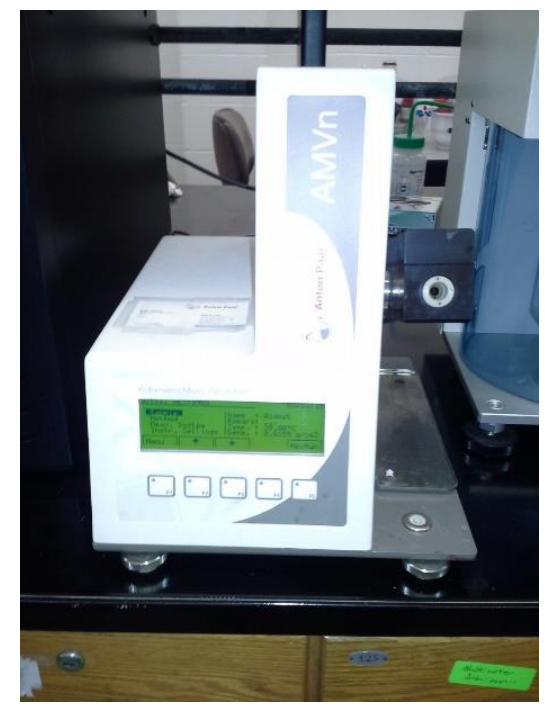

Figure 24: Anton Paar AMVn Automated Microviscometer 


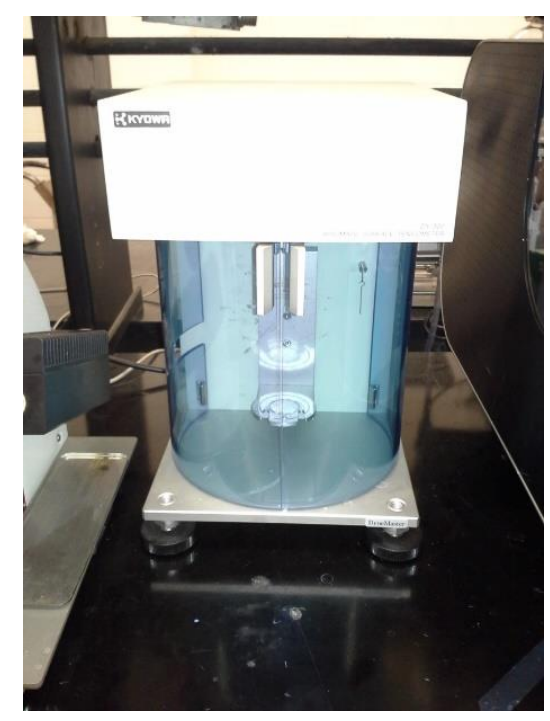

Figure 25: Automatic Surface Tensiometer

Once the cartridge was filled and loaded into the printer, the piezoelectric printhead voltage, frequency of drops, printhead temperature, drop spacing, printed pattern, substrate temperature, and piezoelectric printhead waveform was altered to get the ink to print and the appropriate coverage and layer thickness. Generally, a simple waveform with a positive voltage applied followed by a smaller negative voltage would generate a drop for most inks and was the last thing to be altered after all other options were exhausted. As temperature increases, the viscosity will decrease potentially allowing for drops to be formed at a lower voltage. Therefore, the voltage was increased to help facilitate drop formation and drop size in the range of $10-40 \mathrm{~V}$. The frequency of drops used in these experiments was $5 \mathrm{kHz}$ but could be altered from 1-25 kHz.

The drop spacing, substrate temperature, and printed pattern were altered to develop the appropriate layer which was based on thickness, coverage, and uniformity. The drop spacing played a large role in the coverage and uniformity. If the drop spacing was too wide, the dried product would visibly show drops and have a very low uniformity. While if the drop spacing was too close, the layer produced would be too 
thick. The drop spacing could be altered from 5 to 30 microns. The printed pattern was used to mitigate some of these problems by doing multiple layers and doing alternating rows as the pattern was completely customizable. The substrate temperature played a large role in the drying of the ink which could be altered from room temperature to $60^{\circ} \mathrm{C}$.

\subsubsection{Deposition of Quantum Dot Layer via Drop Casting}

The drop casting of the quantum dot layer procedure was similar to the spin coating procedure. A quantum dot solution was created and a cleaned glass slide was masked using scotch tape to create a known area for the device. A micropipette was then used to deposit $200 \mu \mathrm{L}$ of the solution onto the masked area. The slide was then placed into the vacuum oven over night at $80^{\circ} \mathrm{C}$ and $15 \mathrm{~mm} \mathrm{Hg}$ absolute. If multiple layers were desired, the drying time of one hour was used between layers and after the last layer, set in the oven overnight.

\subsubsection{Deposition of Cathode}

Two methods for deposition of the cathode were undertaken to produce a uniform, continuous conductive layer. One method involves the sputtering of gold onto the device. The other involves the thermal vapor deposition of aluminum and gold onto the device.

The gold sputtered layer was utilized the most because of its accessibility and ease of use. A photo is shown in Figure 26. The equipment used was the SPI-Module Sputter Coater, model 11430. The sample was placed in the vacuum chamber and the

pressure was reduced to $2.5 \times 10^{-5}$ mtorr. The chamber was then purged with argon and 
the pressure reduced back to operating conditions. The power source was then switched on and the argon flow rate was altered to reach an operating current of $5-10 \mathrm{~mA}$ for 4 minutes. Then the operating current was increased to $18 \mathrm{~mA}$ for 8 minutes. Surface thickness studies and current testing was done to ensure an adequate thickness and electrical resistance was reached. The equation given by the manual for determination of the layer thickness is provided in Equation 2. An example of the process is shown in Figure 27.

$$
d=K I V t
$$

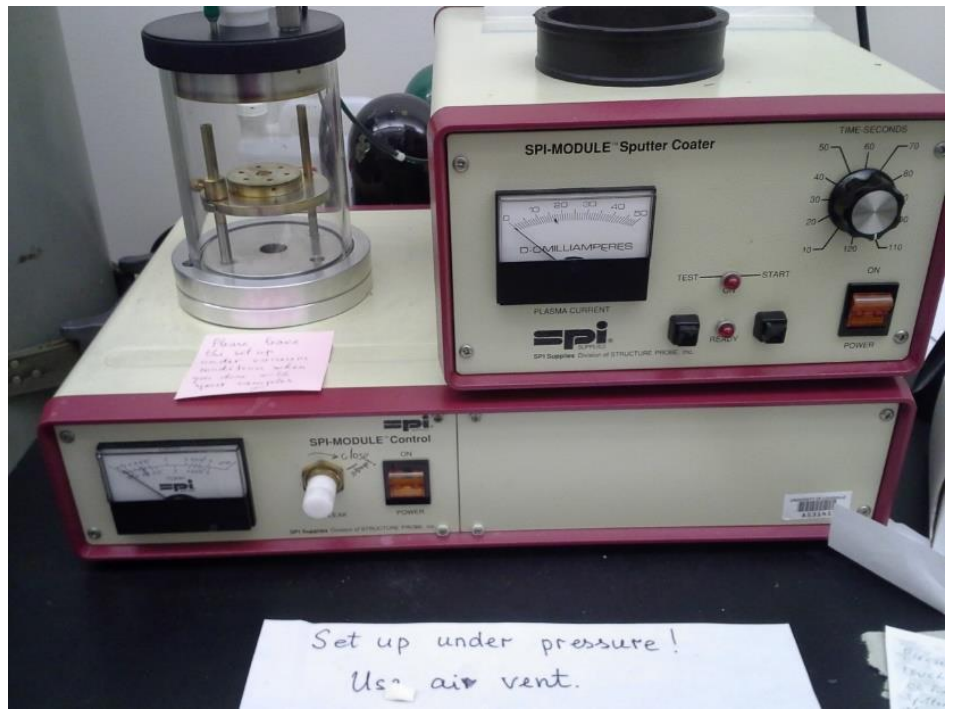

Figure 26: SPI-Model Sputter Coater Model 11430 


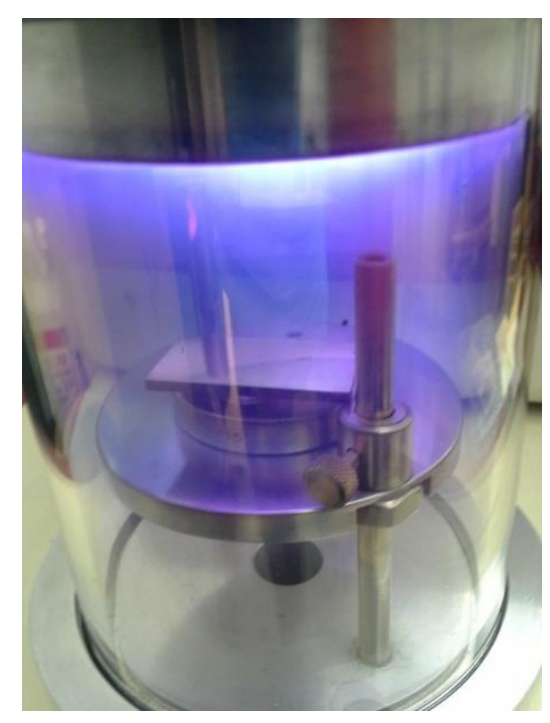

\section{Figure 27: Example of Sputtering Process}

The other method involved the use of a thermal evaporator to deposit aluminum and gold onto the top of the device as the cathode. Multiple device samples were secured to a metal mask before being placed into the thermal evaporator. The mask, shown in Figure 28, had two (2) circular holes of 0.5 inch diameter roughly 0.75 inches apart, center-center, which created two circular deposits of metal onto each slide. The device samples were then placed into the top of the reactor and the pressure was reduced to $2.5 \mathrm{x}$ $10^{-5}$ millitorr before slowly ramping the current up to roughly 25 amperes. The inside of the evaporator needed to be cleaned before each run to monitor the evaporation rate and control the ramping of the current. Examples of aluminum deposited onto samples to create devices are shown in Figure 29. A picture of the thermal evaporator is provided in Figure 30. 


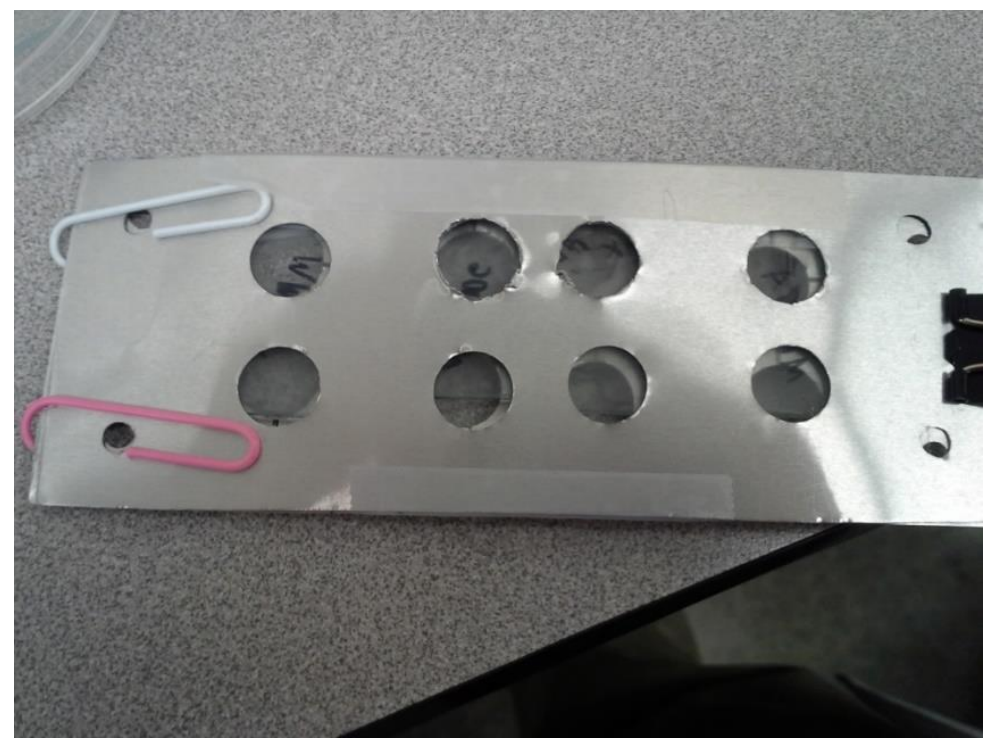

Figure 28: Mask for Thermal Evaporator

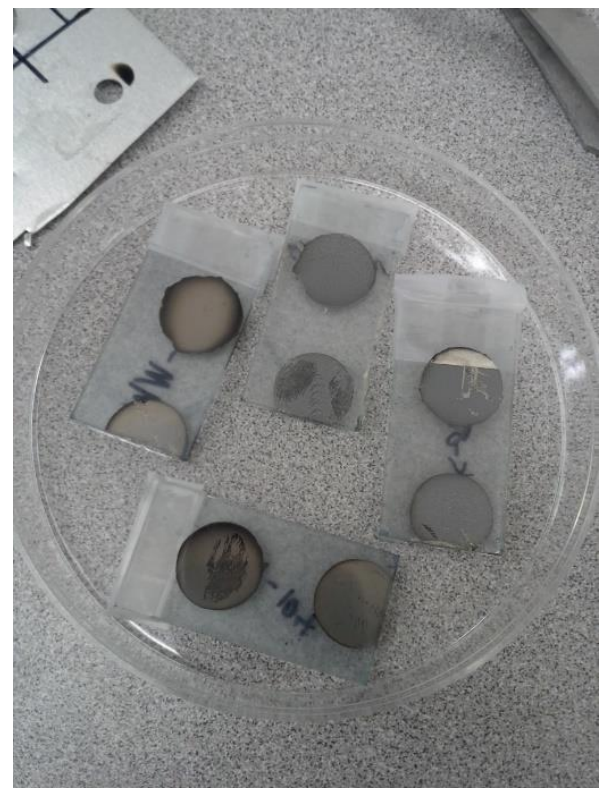

Figure 29: Examples of Aluminum Deposited onto Sample Device 


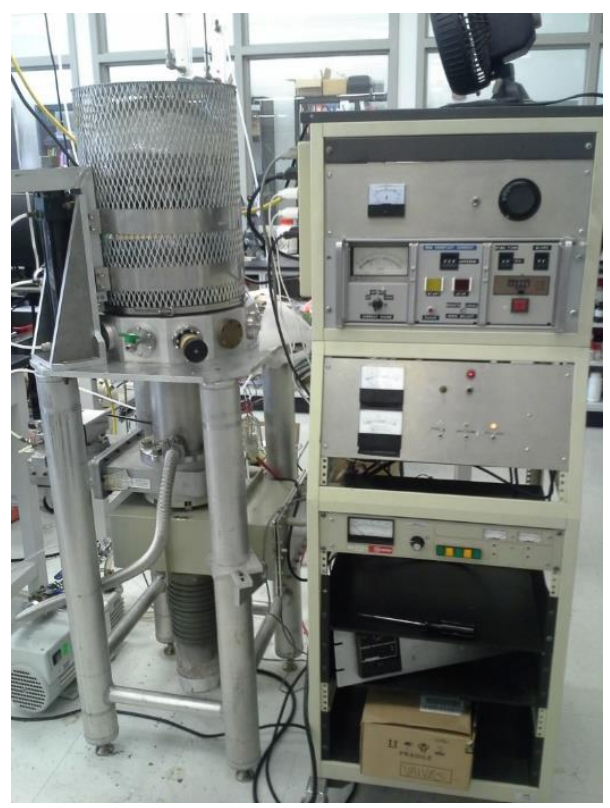

Figure 30: Thermal Evaporator

\subsubsection{Device Testing and I-V Curve Generation}

The constructed and standard devices were tested using a Keithly 2401 SourceMeter interfaced with LabView 7.1. The graphical interface is displayed in Figure 31. Although LabView constructed an I-V curve, the data was saved in a .txt and required importing into an Excel file for further data analysis.

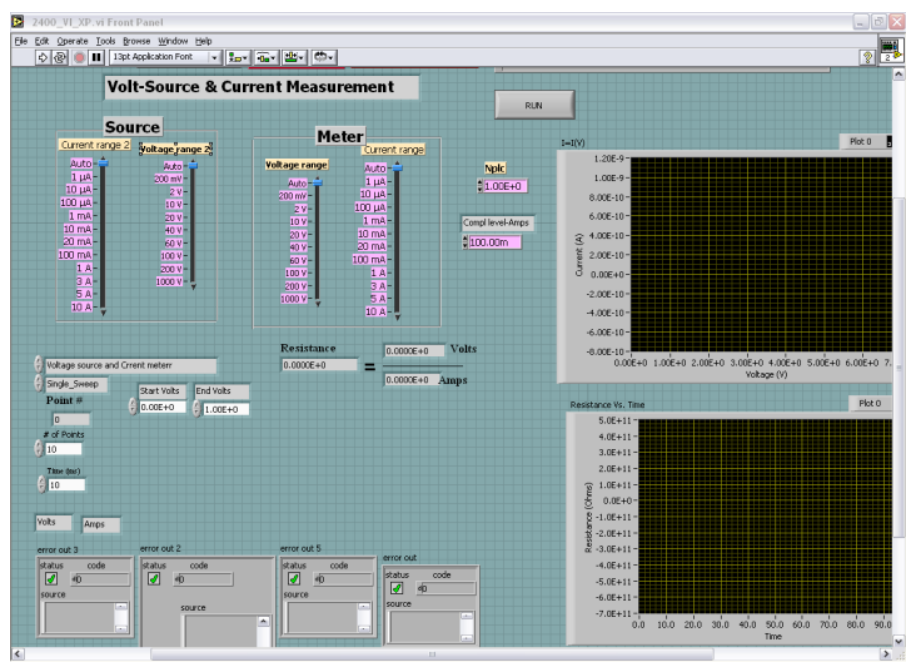

Figure 31: LabView 7.1 Graphical Interface for Device Testing 
After the cathode was deposited, the device was considered ready to be tested. Various methods were used to connect the source meter to the device. The preferred method of connection involved the use of Conductive Silver Paint (SPI Supplies, Item\# 05001-AB SPI) to attach a piece of copper wire to the ITO layer of the device and a separate wire to the gold or aluminum top contact of the device as depicted in Figure 32. The wires were then attached to the source meter. The wire attached to the ITO layer was connected to the anode and, consequently, the top contact was attached to the cathode. The range of voltages over which testing occurred varied depending on the device. There were two apparatus's utilized in the electrical testing of devices. One was called a probing station and is shown in Figure 33. The alternative apparatus was used primarily for housing the samples and is shown in Figure 34.

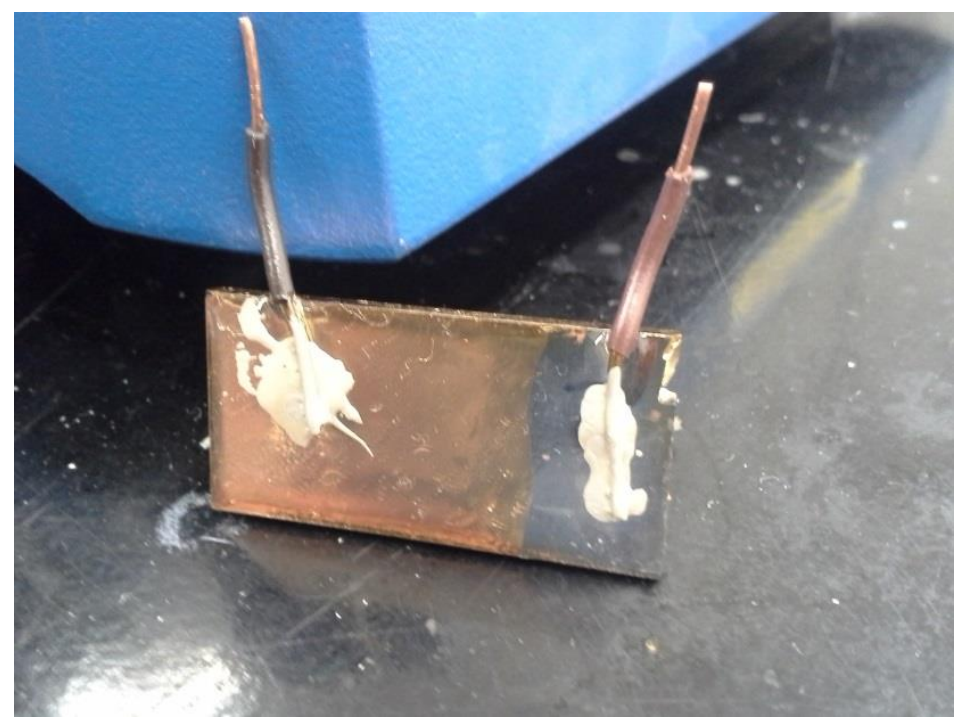

Figure 32: Photograph of Completed Device 


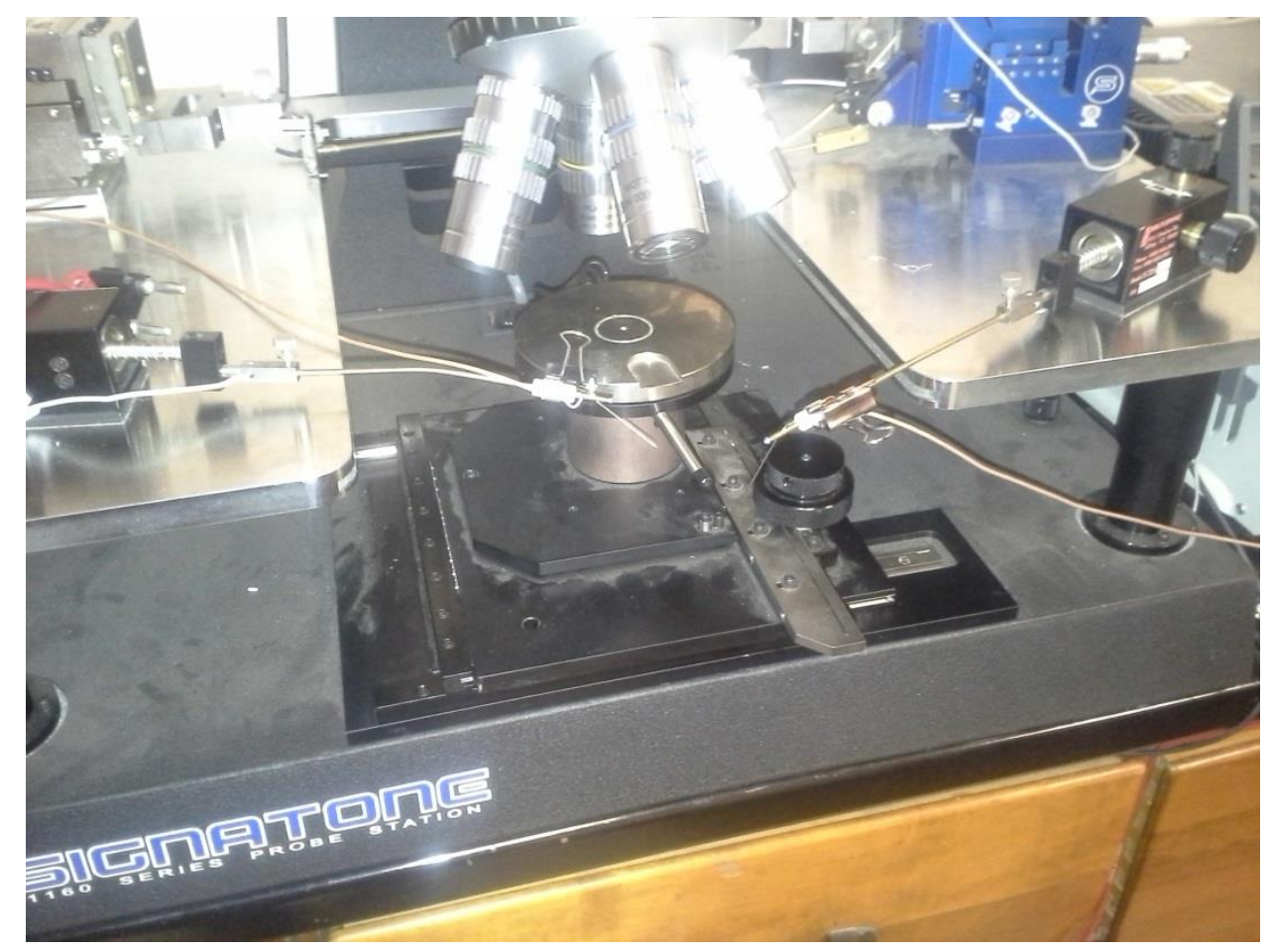

Figure 33: Photograph of Probing Station \#1

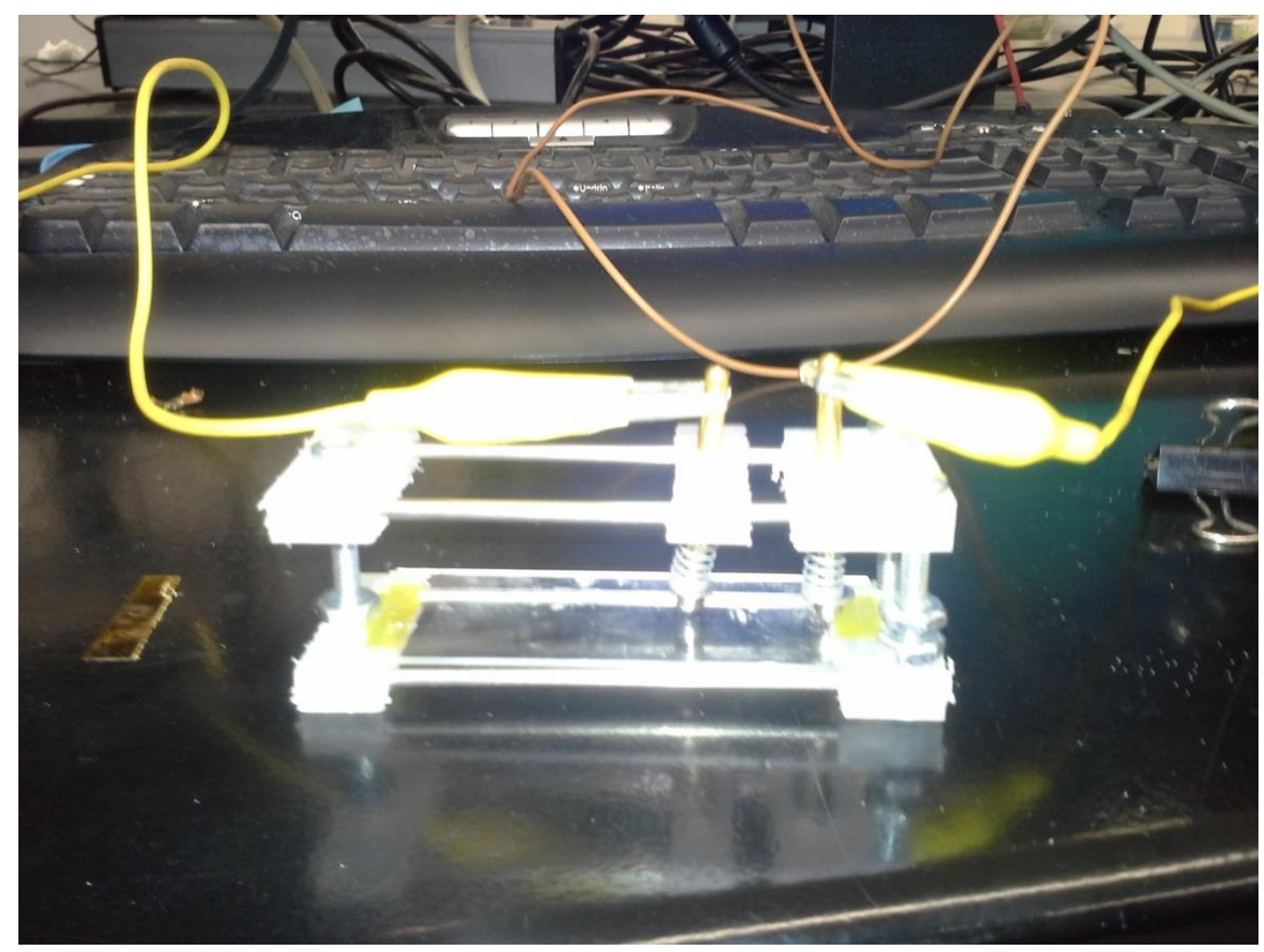

Figure 34: Photograph of Probing Station \#2 


\subsection{Results and Discussion}

\subsubsection{Polymer Layer Deposition and Characterization}

The thickness and average surface roughness of the PEDOT polymer layer were determined using a KLA-Tencor Alpha-Step 500 Surface Profiler. This device operates by dragging a stylus across the surface of the sample and measuring the difference in height as it moves. The stylus has a radius of $12.5 \mu \mathrm{m}$ and the device has a vertical resolution of up to $0.5 \mathrm{~nm}$ but these measurements were run at $2.5 \mathrm{~nm}$ resolution due to the long scan length.

In order to spin coat the PEDOT polymer aqueous solution onto the cleaned ITOcoated glass slide effectively, a more volatile substance was added to ensure a uniform layer. To optimize this process various amounts of ethanol were added to the stock PEDOT polymer aqueous solution. This was decided based on ethanol's miscibility in aqueous solutions and inherently no reactions with the polymer. The compositions were based on the volume of each liquid and each completed layer was carried out using the procedure detailed in the Experimental Section. The following graphs, Figure 35 through 37 are the results from the Surface Profiler on $10 \%, 30 \%$, and $50 \%$ by volume Ethanol to PEDOT:PSS solution. 


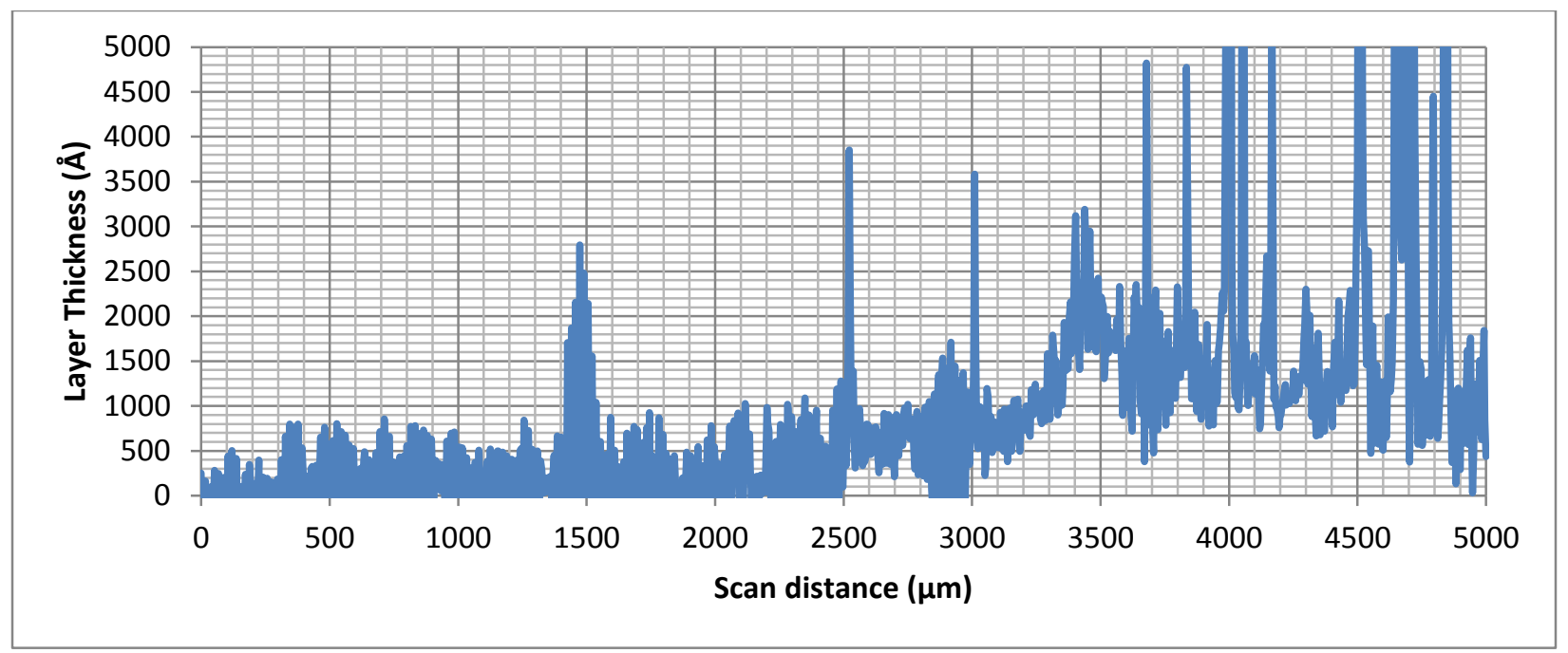

Figure 35: 10\% Ethanol in PEDOT:PSS Soltuion Spin Coated on ITO

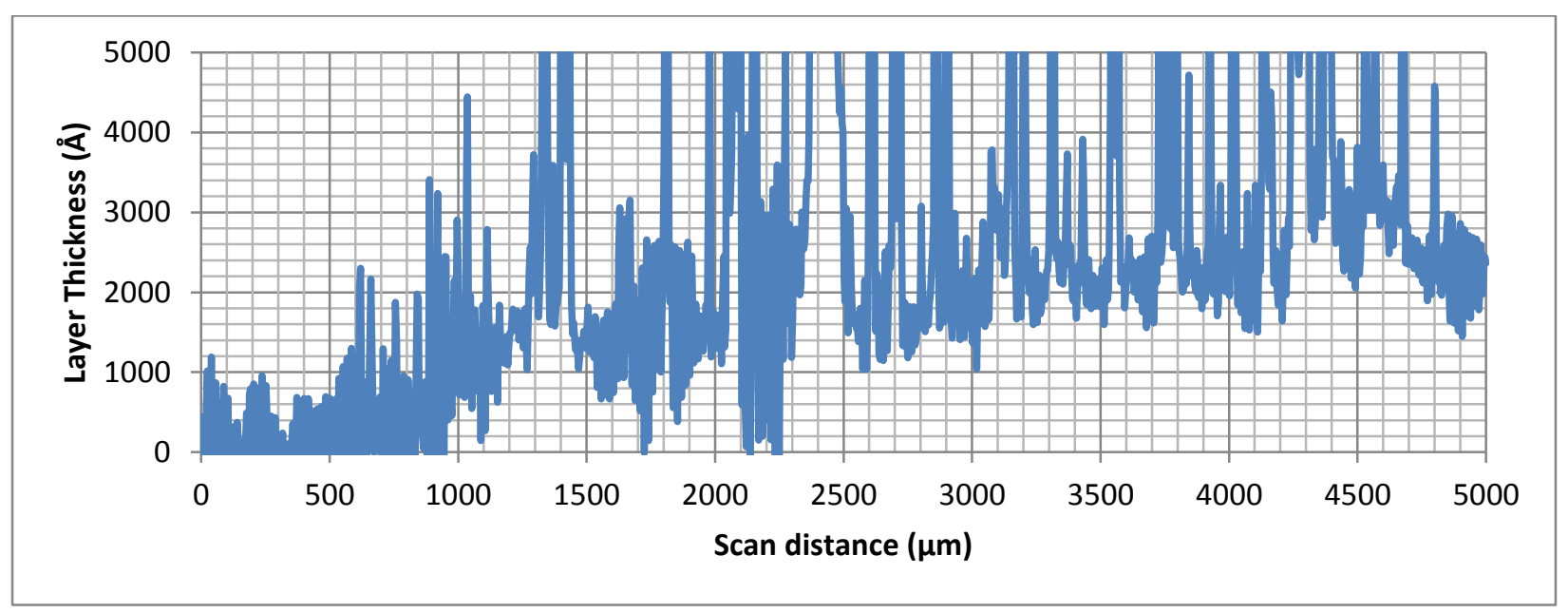

Figure 36: 30\% Ethanol in PEDOT:PSS Solution Spin Coated on ITO

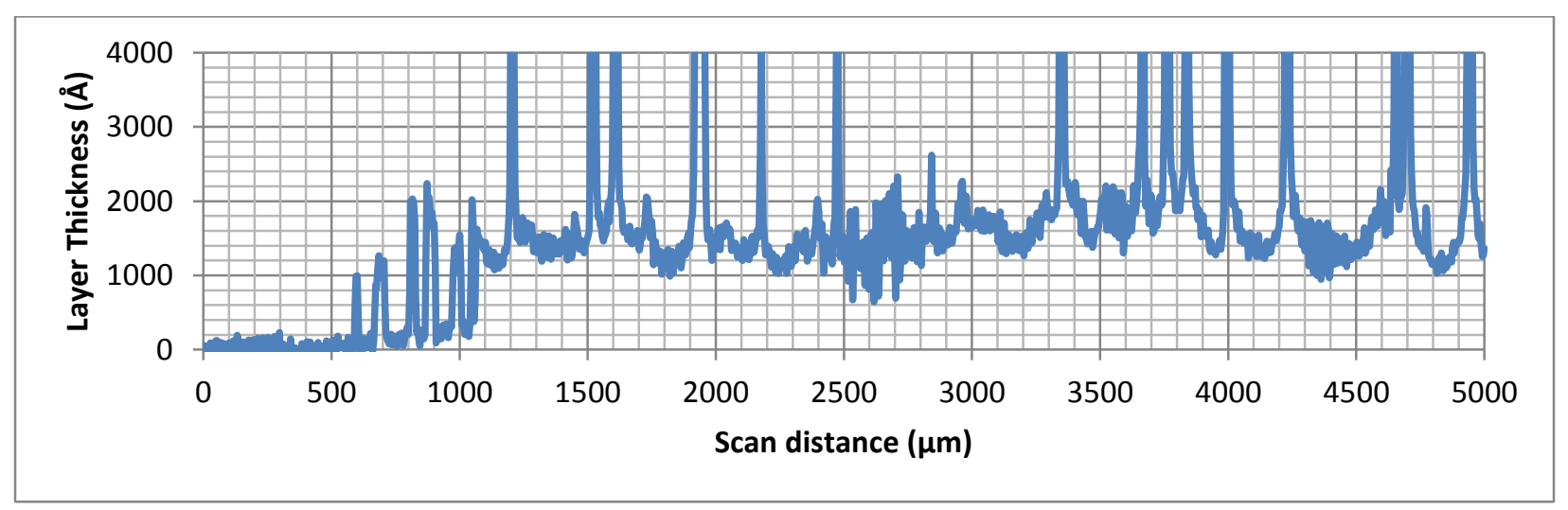

Figure 37: 50\% Ethanol in PEDOT:PSS Solution Spin Coated on ITO 
The $50 \%$ by volume Ethanol in PEDOT:PSS Solution created the best results based on continuity and uniformity of the layer formed. The spikes that are seen are attributed to polymer aggregates and polymer ends which decrease as the percentage of ethanol increases and sonicating the solution but are never completely eliminated. The $50 \%$ spin coated solution had an average surface roughness of 80 . nm including the spikes. If the spikes are not included, the average surface roughness was $24 \mathrm{~nm}$. These calculations were based on Equation 3 and the data points' deviation from $150 \mathrm{~nm}$ thick for the range from $1500 \mu \mathrm{m}$ to $4000 \mu \mathrm{m}$ of the scan distance. The $150 \mathrm{~nm}$ thickness was determined by finding the average thickness without the spikes in thickness. This was done to ensure there were no effects from beginning or end of the scan.

$$
\begin{aligned}
& \text { Average Surface Roughness }=R_{a}=\frac{1}{n} \sum_{i=1}^{n}|y| \\
& \qquad \begin{array}{c}
y=\text { Deviation from } 150 \mathrm{~nm} \\
n=\# \text { of Data points }
\end{array}
\end{aligned}
$$

The following graph, Figure 38, displays the surface profile of Chemical Vapor Deposited PEDOT:TAA Layer. The average thickness was measured to be approximately $30 \mathrm{~nm}$ with an average surface roughness of $86.7 \mathrm{~nm}$ calculated using Equation 3 with deviations from 30nm over the scan range from $500 \mu \mathrm{m}$ to $900 \mu \mathrm{m}$. 


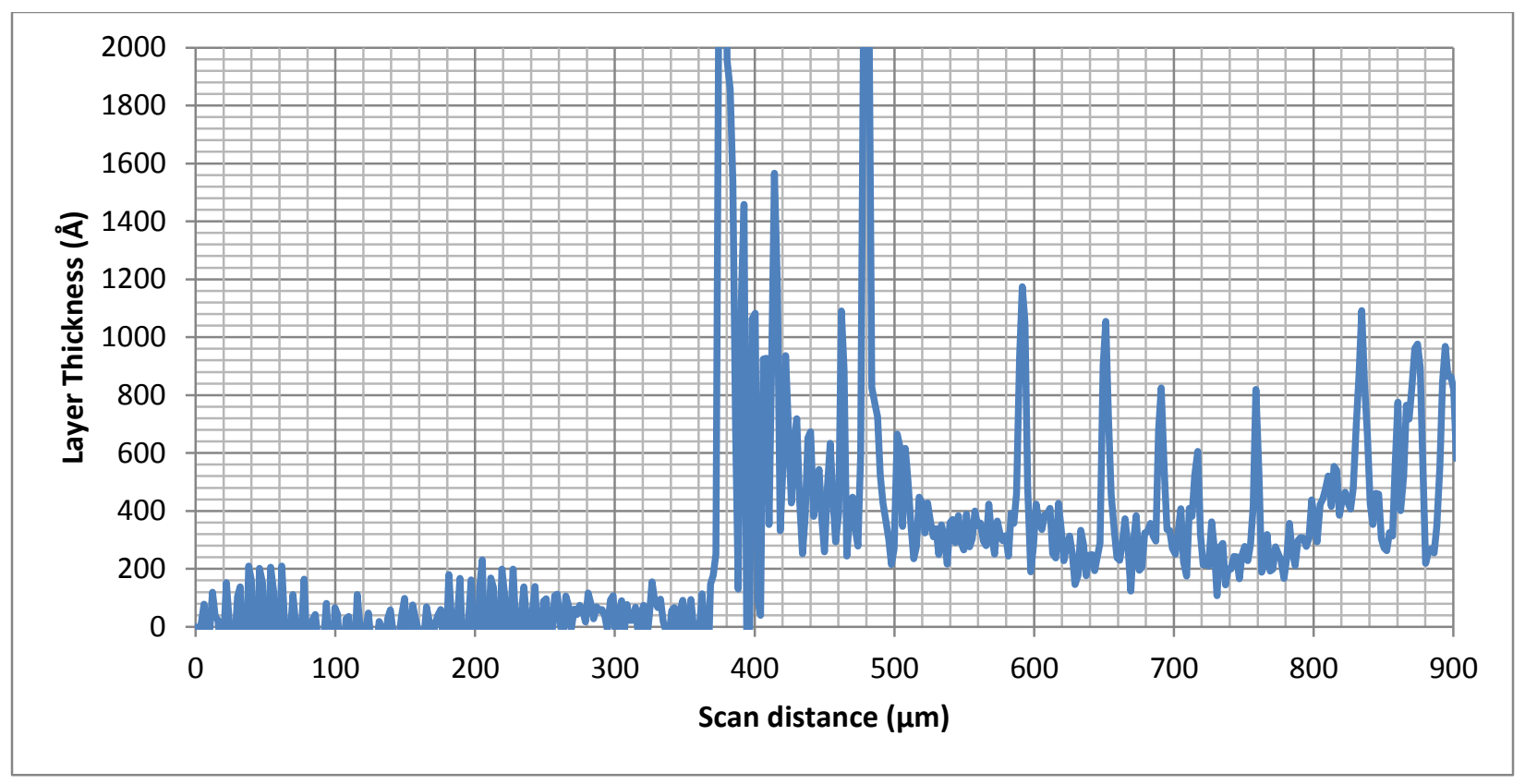

Figure 38: Chemical Vapor Deposited PEDOT Layer on ITO

After the optimized spin coating procedure was well practiced and experience was developed, an Atomic Force Microscopy Image was developed using the XE-100 High Accuracy Small Sample SPM in Non-Contact Mode with a Tap300Al-G Silicon AFM Probe. The AFM utilizes intermolecular forces between the sample and a resonating probe to see differences in height by monitoring the reflection of a laser using multiple photo detectors. The scan area varied, depending on the sample, and the frequency at which each row of data point was taken was $1 \mathrm{~Hz}$.

The AFM images developed are consistent with the surface profiles generated by the Surface Profiler. Figure 39 displays a 3-D depiction of the scan gathered from the spin coated 50\% ethanol in PEDOT:PSS solution. There are clearly still spikes in thickness but the AFM analysis has allowed the generation of a more comprehensive understanding of the surface. A complete analysis of the entire surface from the AFM image is displayed in Table 18. The average surface roughness, $R_{a}$, was $9.65 \mathrm{~nm}$. The 
root mean square surface roughness, $\mathrm{R}_{\mathrm{q}}$, was $13.155 \mathrm{~nm}$. This value takes into account the negative values as well, meaning that this value is slightly more descriptive as there are some negative deviations from the typical surface. The maximum height profile of the sample, $\mathrm{R}_{\mathrm{pv}}$, was 91.62 which is the difference between the lowest point and highest point. The skewness of the surface roughness, $\mathrm{R}_{\mathrm{sk}}$, was $1.473 \mathrm{~nm}$ which describes the tendencies of the deviations, either positive or negative. The kurtosis of the surface roughness, $\mathrm{R}_{\mathrm{ku}}$, was $5.163 \mathrm{~nm}$. This term describes how well the data is focused on a peak.

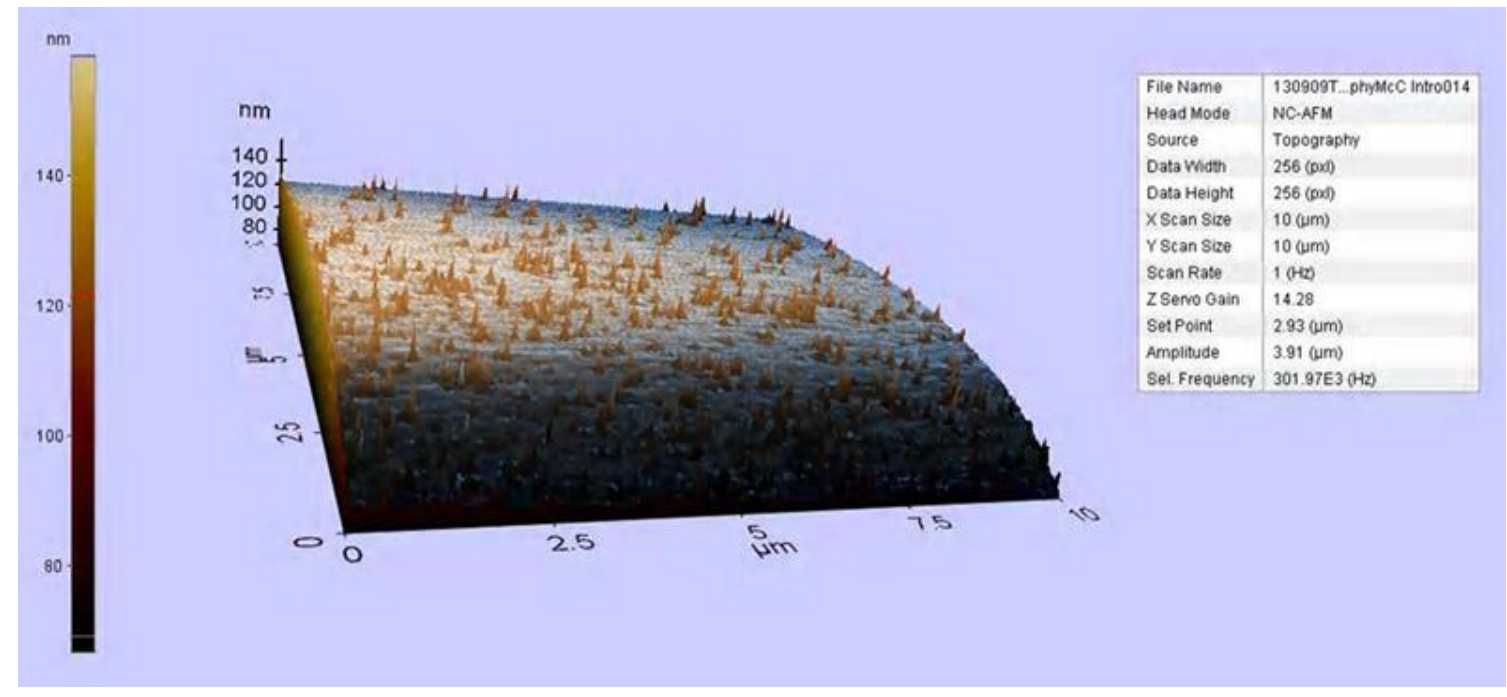

\section{Figure 39: AFM Image of 50\% Ethanol in PEDOT:PSS Solution Spin Coated on ITO Glass Slide}

Table 16: AFM Analysis of 50\% Ethanol in PEDOT:PSS Solution Spin Coated

\begin{tabular}{|l|l|l|l|l|}
\hline $\operatorname{Rpv}(\mathrm{nm})$ & $\mathrm{Rq}(\mathrm{nm})$ & $\mathrm{Ra}(\mathrm{nm})$ & $\mathrm{Rsk}$ & $\mathrm{Rku}$ \\
\hline 91.624 & 13.155 & 9.654 & 1.473 & 5.163 \\
\hline
\end{tabular}

Due to the roundness of the scan developed from an uneven sample substrate, a smaller area of the scan was analyzed to possibly yield a better representation of the surface. The analysis was taken of roughly the middle third of the above AFM image and is displayed in Table 19. 
Table 17: AFM Analysis of 50\% Ethanol in PEDOT:PSS Solution - Smaller Area

\begin{tabular}{|l|l|l|l|l|}
\hline $\operatorname{Rpv}(\mathrm{nm})$ & $\mathrm{Rq}(\mathrm{nm})$ & $\mathrm{Ra}(\mathrm{nm})$ & $\mathrm{Rsk}$ & $\mathrm{Rku}$ \\
\hline 38.295 & 4.594 & 3.886 & -0.192 & 2.847 \\
\hline
\end{tabular}

To compare the spin coated sample to the chemical vapor deposition sample, an AFM image was generated and an analysis was done on the chemical vapor deposited PEDOT slide which is displayed in Figure 40. The AFM image of the CVD sample is clearly rougher than the spin coated slide. The analysis is shown in Table 20.

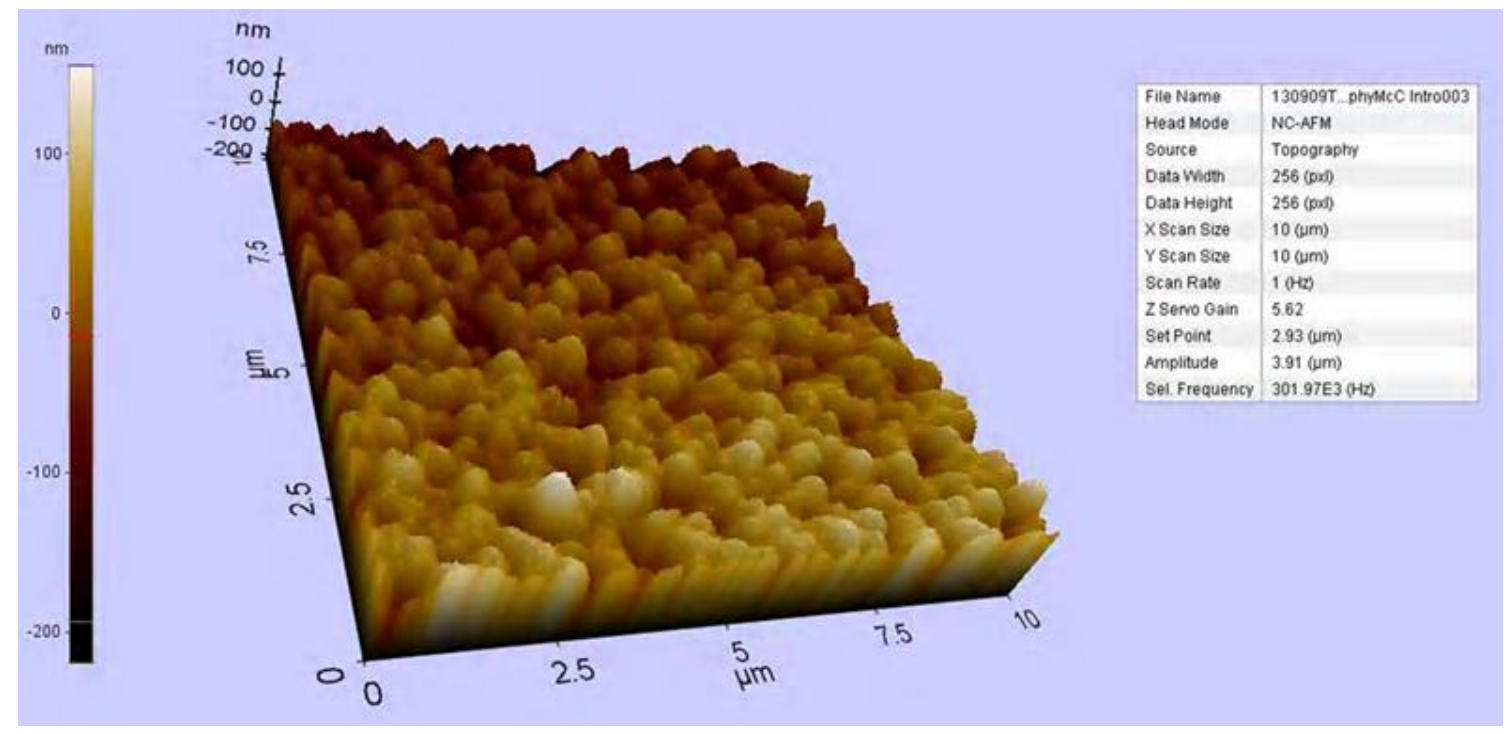

Figure 40: 3-D AFM Depiction of Chemical Vapor Deposited PEDOT:TAA

Table 18: AFM Analysis of Chemical Vapor Deposited PEDOT:TAA

\begin{tabular}{|c|c|c|c|c|}
\hline $\operatorname{Rpv}(\mathrm{nm})$ & $\mathrm{Rq}(\mathrm{nm})$ & $\mathrm{Ra}(\mathrm{nm})$ & $\mathrm{Rsk}$ & $\mathrm{Rku}$ \\
\hline 374.886 & 53.528 & 43.566 & 0.232 & 2.726 \\
\hline
\end{tabular}

To ensure that there is a direct comparison for the smaller area analysis, an analysis of a smaller area of the same area was completed and shown in Table 21. 
Table 19: AFM Analysis of Chemical Vapor Deposited PEDOT:TAA - Smaller Area

\begin{tabular}{|l|l|l|l|l|}
\hline $\mathrm{Rpv}(\mathrm{nm})$ & $\mathrm{Rq}(\mathrm{nm})$ & $\mathrm{Ra}(\mathrm{nm})$ & Rsk & $\mathrm{Rku}$ \\
\hline 237.423 & 38.039 & 30.157 & 0.553 & 3.209 \\
\hline
\end{tabular}

To compare the two different methods of polymer deposition and polymer composites, the spin coated layer has a $R_{a}$ of $3.886 \mathrm{~nm}$ while the chemical vapor deposited layer has one of $30.157 \mathrm{~nm}$ using the smaller area of both techniques. The layer with the smaller average surface roughness should allow for a monolayer of quantum dots to form which is what is preferred. The roughness of the chemical vapor deposited layer could lead to increased risk of short circuiting and device degradation. A positive, larger $\mathrm{R}_{\mathrm{sk}}$ is also important to note in this analysis as it describes how the data is distributed from the ideal surface. Since this number is positive and larger for the spincoated layer, it could be deduced that this layer is less likely to contain pin-holes which lead to short circuiting of the device.

The effect of the polymer-quantum dot interface on the photoluminescence properties was also analyzed using Photoluminescence Spectroscopy. This was completed by spin coating mercapto-benzoic acid/TOP capped CdSe QD onto one spin coated PEDOT:PSS slide and one CVD PEDOT:TAA slide and a photoluminescence spectroscopy analysis was run. The PL spectrum of the PEDOT:PSS-mercapto-benzoic acid/TOP capped CdSe QD slide is displayed in Figure 41 while the CVD counterpart is displayed in Figure 42. Table 11 and Figure 15 can be referred to as a reference for mercapto-benzoic acid/TOP capped CdSe QD luminescent properties. The spin coated PEDOT:PSS results in a maximum peak shift from $555 \mathrm{~nm}$ to $490 \mathrm{~nm}$ and a FWHM of $110 \mathrm{~nm}$ to $66 \mathrm{~nm}$. While the CVD PEDOT:TAA resulted in a maximum peak shift from 
$555 \mathrm{~nm}$ to $507 \mathrm{~nm}$ and a FWHM of $110 \mathrm{~nm}$ to $229 \mathrm{~nm}$. Clearly, there is a significant difference between the two deposition techniques and/or polymer composites.

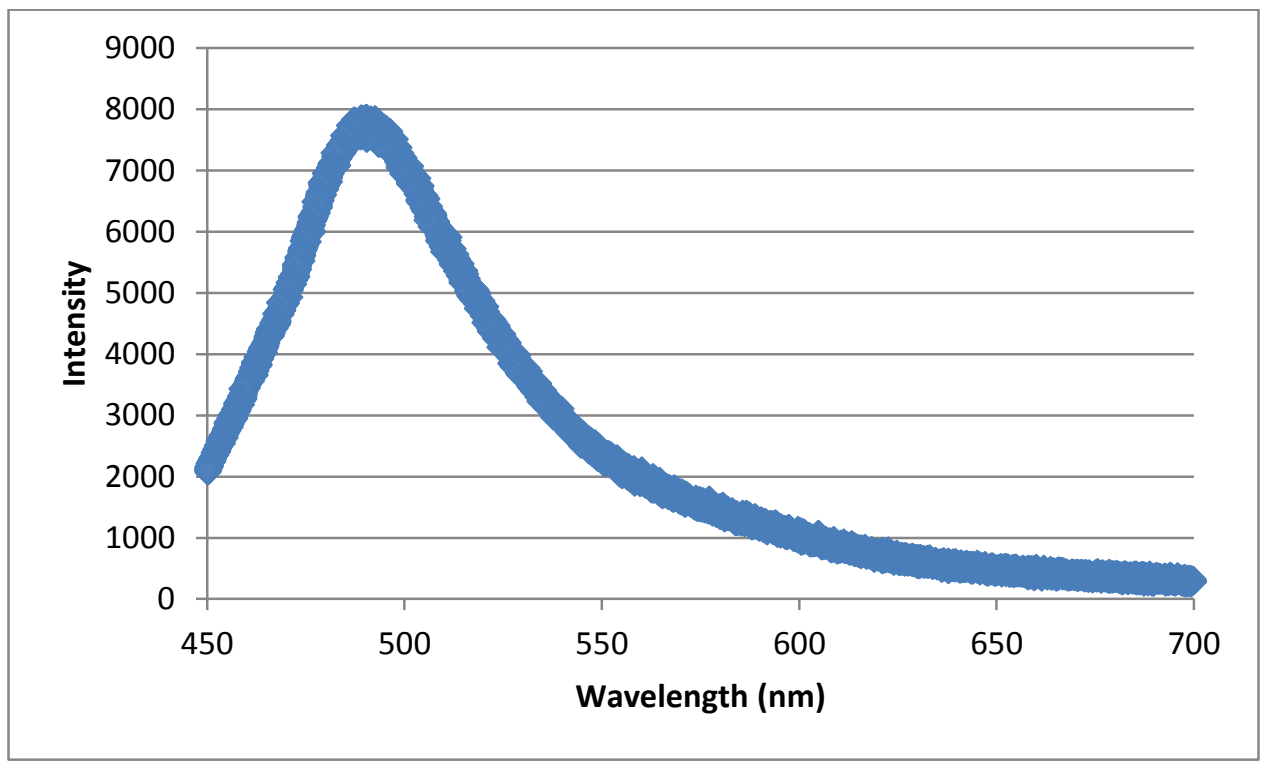

Figure 41: Spin Coated PEDOT:PSS - mercapto-benzoic acid/TOP capped CdSe QD slide PL Spectrum

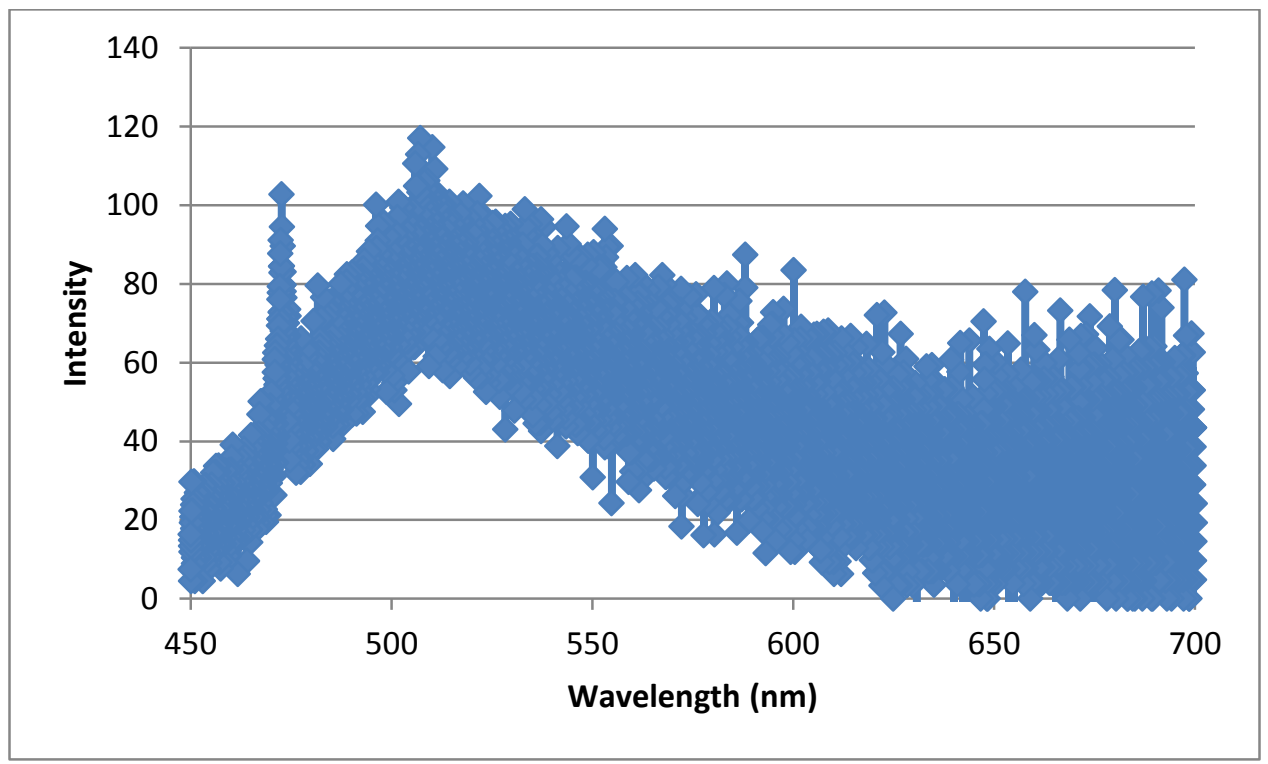

Figure 42: CVD PEDOT:TAA - mercapto-benzoic acid/TOP capped CdSe QD slide PL Spectrum

The spin coated 50\% PEDOT:PSS solution was used in the devices to create the holeinjection layer of the device for multiple reasons. Although the CVD PEDOT:TAA had 
the more optimal layer thickness, the difference was not enough to warrant the introduction of pin holes into the device. The other reason for using the PEDOT:PSS was the PEDOT:TAA appeared to have a photoluminescence quenching effect on the quantum dot which is highly unfavorable.

To better understand why the spikes witnessed in Figures 37 and 39 of the PEDOT:PSS were formed, a thermodynamic analysis of the polymer solutions was undertaken. The polymeric solution can be considered very dilute, $<2 \%$ by weight which based on a polymer with an average molecular weight of 1,000-100,000 g/mol and a solvent with a molecular weight of $300 \mathrm{~g} / \mathrm{mol}$, yields a molar ratio of $0.0007-0.07 \%$, respectively. These values are based on typical average molecular weights of polymers and an extremely large organic solvent to demonstration a more concentrated scenario. Important to note, it is highly advantageous to use a highly volatile species, such as, chloroform, as the solvent to create a uniform film when spin coating. Therefore, it is a justified assumption that the polymer is non-volatile.

This polymer solution can be modeled using liquid-vapor equilibrium models, such as, Modified Raoult's Law. Although the system is a polymeric solution, the solvent is the only component that is of concern at first. For creation of a thin film, it is important that the vapor pressure of the solvent is extremely high. To model this using the Modified Raoult's Law, the Flory-Huggins Theory for calculating the activity coefficient of the polymeric solutions will be utilized. This theory dictates that there is no volume change upon mixing and no excess entropy. Since the solution is very dilute, these assumptions are consistent with the polymeric solution. [33] The underlying equation is Modified Raoult's Law which is modified by the addition of an activity coefficient as shown in 
Equation 4. It is also at a constant temperature which will dictate what the saturation pressure will be.

$$
P * y_{1}=P_{1}^{s a t} x_{1} \gamma_{1}
$$

As mentioned before, the polymer is considered nonvolatile and the only component this equation is in reference to is the solvent, such as chloroform, water, or some other liquid. The activity coefficient, $\gamma$, will be calculated using the Flory-Huggins Theory as shown in Equation 5.[33] As the above equation shows, to increase the volatility of the solvent, the activity coefficient needs to be maximized.

$$
\ln \gamma_{1}=\ln \left(1-\left(1-\frac{1}{r}\right) \Phi_{2}^{*}\right)+\left(1-\frac{1}{r}\right) \Phi_{2}^{*}+\chi \Phi_{2}^{*}
$$

Based on Equation 5, there are some parameters that are used to help select the appropriate solvent and chain length of the polymer. Many polymers can be purchased at varying chain lengths from 10k to 1000k repeat units. Equation 6 was developed based on the assumption that the solvent molecule and the polymer repeat unit are similar molecules and would have the same type and magnitude of intermolecular forces acting on each other. As the chain length, $r$, decreases, the activity coefficient approaches unity and the solution becomes ideal. The other variable in the equation is the volume fraction. At the beginning of the spin coating, the volume fraction of the polymer is orders of magnitude smaller than the volume fraction of the solvent. The far right term in the equation attempts to correct for some of the questionable assumptions that were made, such as, the type and magnitude of the intermolecular forces are the same between the solvent and the polymer verses the forces each substances has upon itself. The following equation shows the volume fraction for the polymer when the volume of the monomer of the polymer is close to the volume of the solvent: 


$$
\Phi_{2}=\frac{r N_{2}}{N_{1}+r N_{2}}
$$

Where $\mathrm{N}_{\mathrm{i}}$ is the number of molecules of species $\mathrm{i}$. In the case of spin coating conjugated polymers, the solvent molecule is usually small so as to promote volatility and fast drying while a typical conjugated polymer repeat unit is made of one or more aromatic double bonded molecules. Therefore, to correct for the volume difference between a solvent molecule and polymer repeat unit and difference in the type of intermolecular forces, the Flory-Huggins interaction parameter, $\chi$, based on the intermolecular forces between the molecules, is used:

$$
\chi=\frac{w}{k T}
$$

Where $\mathrm{w}$ is the interchange energy which is the sum of the interaction and repulsion forces between the repeat unit and solvent molecules. If $r$ was set equal to the ratio of the molar volumes of polymer and solvent, the $\Phi$ would become identical to the volume fraction in the Scatchard-Hildebrand theory which would probably better describe the system. The interaction parameter is then defined as Equation 8:

$$
\chi=\frac{v_{1}}{R T}\left(\delta_{1}-\delta_{2}\right)^{2}
$$

Where $\delta$ is the Hildebrand solubility parameter for the component. For solubility parameters that are not known, Equation 9 can be used:

$$
\delta=\left(\frac{\Delta U_{v a p}}{v}\right)^{\frac{1}{2}}
$$

For the system described, the difference between the two solubilities will be considerable meaning the solvent's solvation abilities are not high but this is not important for phase separation of the polymer and quantum dot. Therefore, there is an upper limit where partial miscibility occurs because $\chi$ is positive. To counter act this possible solubility 
problem, the temperature can be raised above the upper critical solution temperature (UCST). The critical volume fraction, $\Phi^{\mathrm{c}}$, and interaction parameter, $\chi^{\mathrm{c}}$, can be calculated at low temperatures using Equations 10 and 11.

$$
\begin{aligned}
& \chi c=\frac{1}{2}\left(1+\frac{1}{\sqrt{r}}\right)^{2} \\
& \Phi_{2}^{* C}=\frac{1}{1+\sqrt{r}}
\end{aligned}
$$

Figure 43 gives a good visual to how the solution will behave at the critical parameters at the UCST.

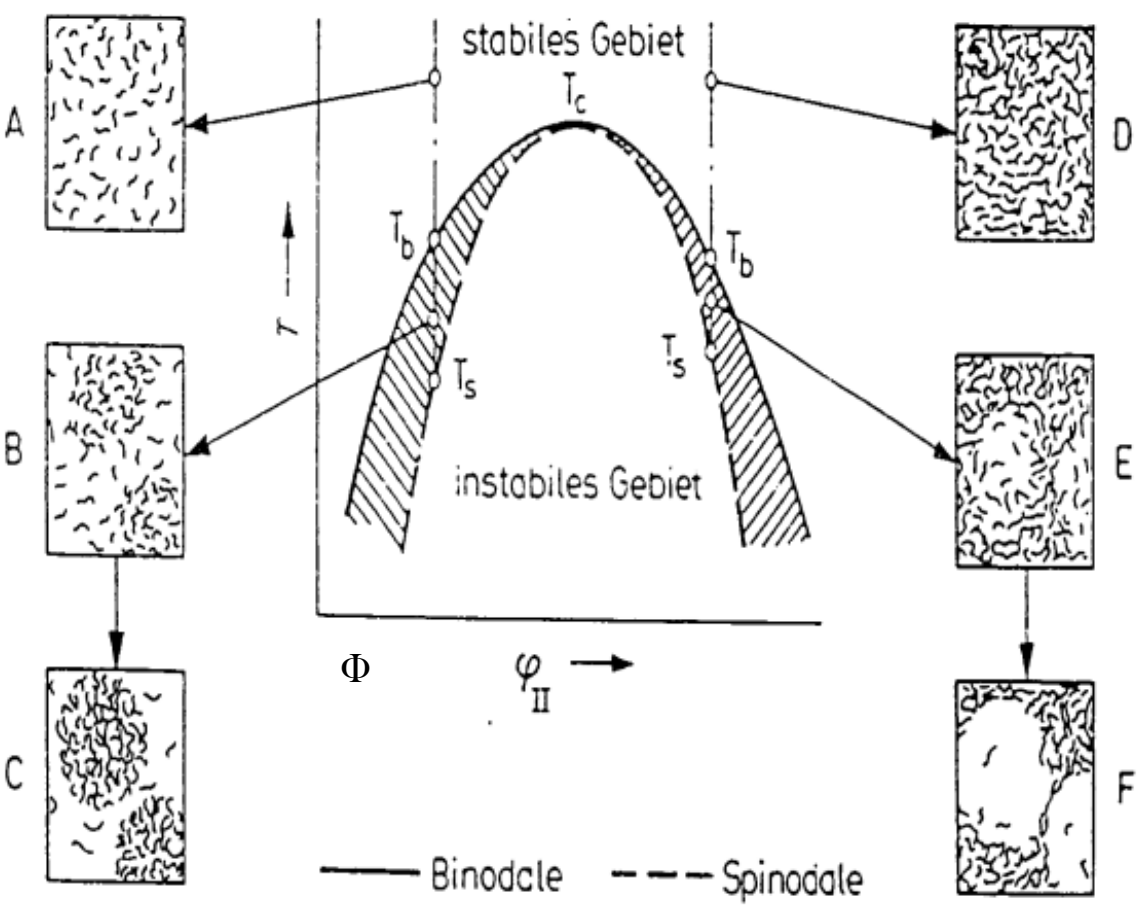

Figure 43: Schematic phase diagram for solutions of a molecularly uniform polymer. Polymer lean phase (sol): A - stable, B - metastable, $\mathrm{C}$ - unstable and segregation of a gel phase. Polymer rich phase (gel): D - stable, E - metastable, F unstable and segregation of a sol phase.[34]

One of the downfalls to the simple Flory-Huggins Theory is that it only predicts positive deviations from ideality. Partial miscibility occurs at higher temperatures which is a negative deviation from ideality and is known as the lower critical solution 
temperature (LCST). As the molecular weight increases, the difference between the LCST and UCST typically decreases. The LCST can be calculated using some complex empirical correlations based on composition and temperature for the Flory-Huggins interaction parameter, $\chi$, developed by Qian and coworkers and published by Bae et al. [35]

Only solutions that are stable for entire range of volume fractions create uniform, continuous thin films. This is because of the different phases that are formed in these nonhomogeneous states above and below the LCST and UCST. If a discontinuity in the film called a pinhole occurs, a short through the layer will happen and render the device useless. In practice, a stable gel phase (shown in Figure 43) is the most important part because it is the last phase occurring before the layer is fully formed. Therefore, a low molecular weight must be considered to ensure a stable solution into the gel phase.

Unfortunately, these calculations are only a ballpark answer and cannot exactly predict the behavior of all systems. In most cases, there are many more parameters that must be taken into account or at least considered such as reactions between the two molecules, solvation of the polymer molecule and other non-idealities such as temperature and pressure. In reality, experimental data must be collected to predict the behavior of a specific system and use the correlations developed such as Bae et al.[35] Through this analysis, it was found that temperature and chain length are also important factors in promoting phase separation. As temperature decreases, the solution will become unstable and phase separation will occur. The chain length of the polymer can control when the phase separation will occur as the solvent evaporates. 
The last step of this process involves the heating of the polymer covered ITOglass slides in a vacuum oven for an extended period of time. This step is to ensure the complete evaporation of the solvent molecule by providing the sample with excess energy to release the solvent molecule from whatever bond it may have with the polymer or itself within the sample. This heating step also has the added benefit of promoting crystallization in the polymer in the layer which can reduce charge traps and increase efficiency. The most basic equation that will be used for this analysis will be Equation 12. Also, for a process to be considered favorable or spontaneous, the Gibbs free energy, $\mathrm{G}$, must be negative where $\mathrm{H}$ is enthalpy, $\mathrm{S}$ is entropy, $\mathrm{T}$ is temperature, and $\Delta$ is the function of change.

$$
\Delta G=\Delta H-S \Delta T
$$

Refreshing the assumption that the solvent is not a similar molecule to the repeat unit of the polymer, there must be some thermodynamic qualities that help increase the solubility of the polymer. In 1949, Paul Flory did a theoretical study of this phenomenon using statistical thermodynamics. [36] Although the details of his work are not in the scope of this paper, the concept of crystallites in the solution is in the scope and can help describe solubility in the solution. Crystallites can form in polymer solutions and even polymer melts and they are basically small areas of crystalline polymer. The size of the crystallites is dependent on the length of the chain, heat of fusion, and dilution of solution.

The length of the polymer chain dictates the upper most limit of the size of the crystallites and affects the heat of fusion while the dilution of the solution will affect the probability of polymer molecules coming into contact with one another. Ultimately, the 
combination of these things result in the enthalpy of fusion being more exothermic (negative) than the entropy of fusion (positive for crystallization) at this decreased temperature at a specific dilution of the solution. As the solution becomes more concentrated, the solvent molecules can inhibit the crystallization less and less and the crystallinity becomes greater as it becomes more thermodynamically stable but can never reach $100 \%$ crystallinity, at least using this approach.

The fringed micelle model was first conceived by Hermann, Gerngross and Abitz[37] but a similar, relevant depiction is showcased in Figure 44. As is shown by the combination of the images, there is only a small amount of crystalline phase which is dependent of the dilution on the solution as modeled by the graph on the right. As the spin coating occurs and the solution becomes more concentrated, those crystalline regions grow.
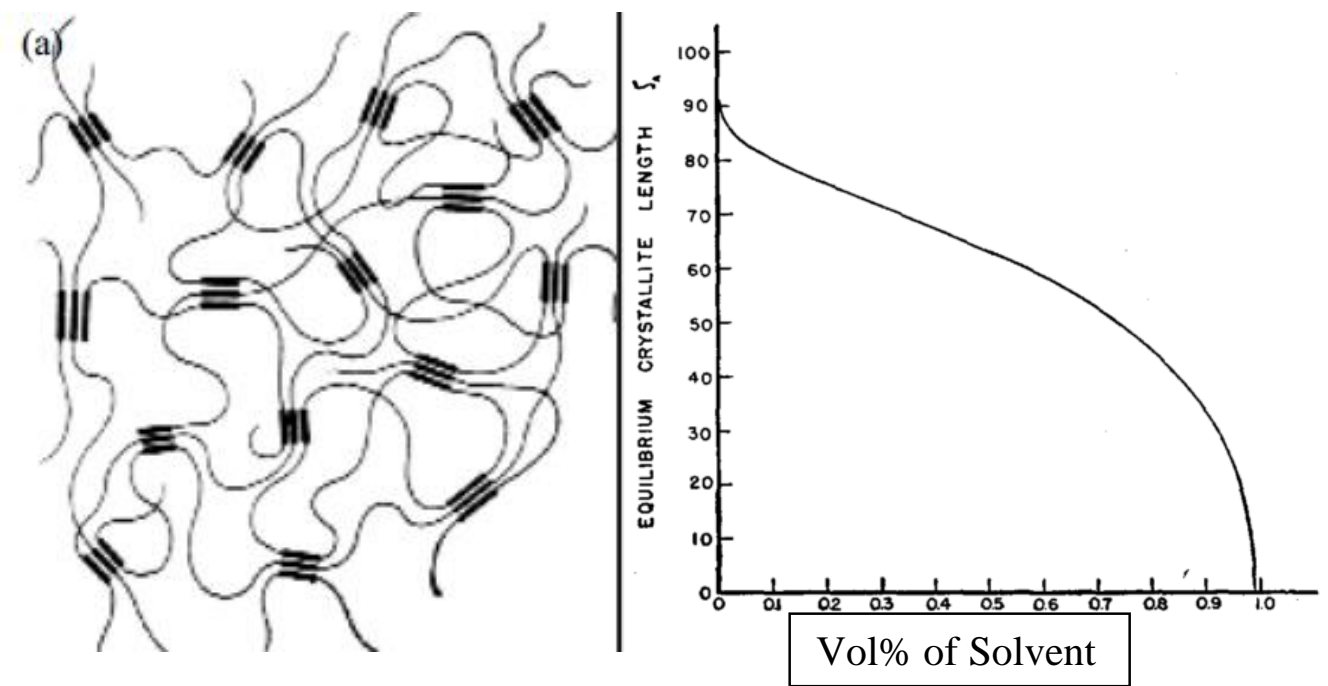

Figure 44: (Left) Depiction of the crystallization of a thermoreversible gel [38] (Right) Equilibrium crystallite length as a function of dilution[36]

Due to the nature of spin coating, the graph of the crystallinity is not fully reached as the concentration of the solution is increased so quickly that it is quenched at some point in-between. Also, Flory made some assumptions that are in contradiction to the 
actual system. One key assumption is that the solvent molecules are similar to the repeat unit of the polymer. This error could have an effect on the results of the crystallinity but should not affect the nature of this phenomenon. The flexibility of conjugated polymers is also significantly more rigid than the ideal polymer but with significantly high enough molecular weight, the effect of this should be negligible.

At this point, the heating of the sample helps to give the polymer molecules enough energy to promote further crystallinity. The sample is heated to above the glass transition temperature which is the point at which the polymer has enough energy to move into a more crystalline position. Since the device is a multi-component system, this cannot always be attained. Sometimes, there are other materials that are not stable at the glass transition temperature of the polymer of choice and this step would degrade the device.

\subsubsection{Deposition of Cathode}

The deposition of gold was easily obtained using the sputter coater. A blank was run to ensure that an appropriate resistance for the layer was created. The contacts were bound to the gold using silver paste as the device also used this technique. An I-V curve was generated to analyze the resistance of the layer and is displayed in Figure 45. 


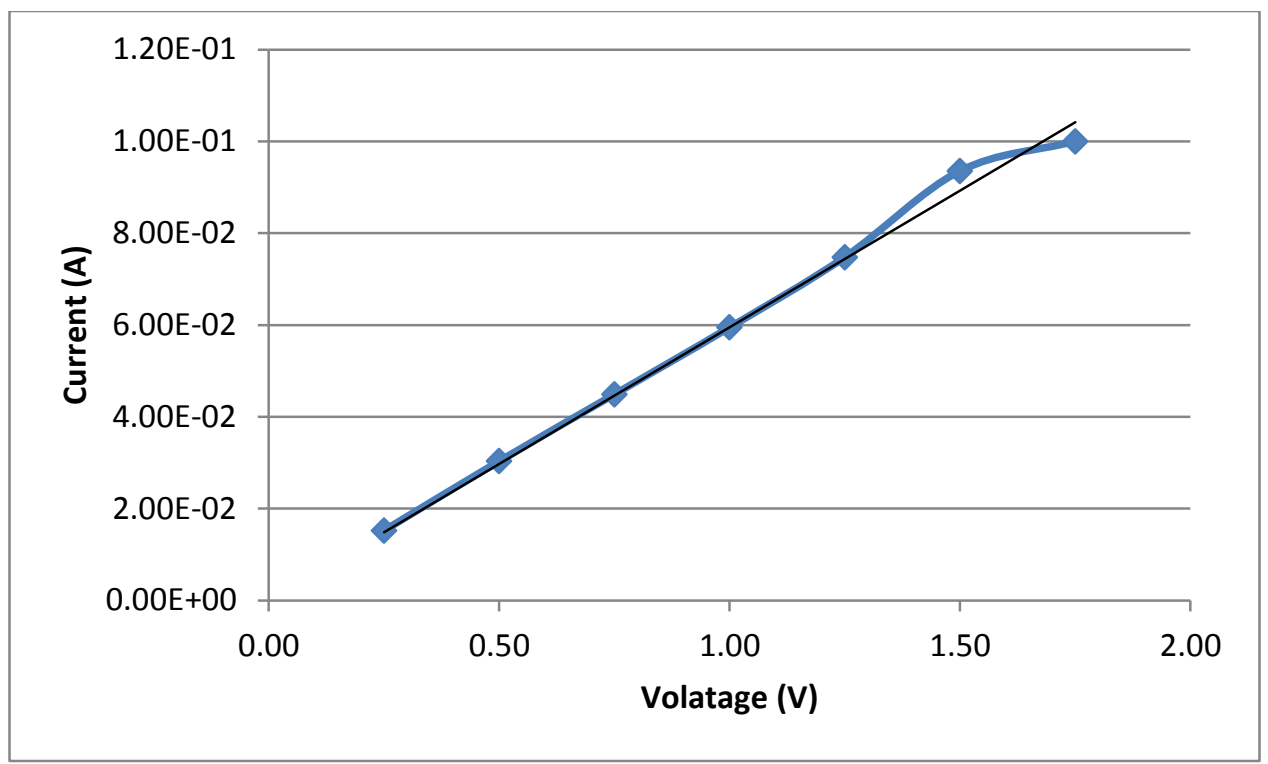

Figure 45: I-V Curve for Gold Sputter Coated on Glass

The slope was then taken and used to find the resistance through the layer by means of Ohm's Law. The resistance was calculated to be $16 \Omega$ which should provide sufficient resistance. A sufficient resistance is one or two orders of magnitude difference between the layers to provide an even charge distribution during operation.

A similar process was carried out for the aluminum layer deposited by thermal vapor. The resultant resistance was $15.5 \Omega$ and should provide the same phenomena for device operation. The I-V curve which was generated for this layer is displayed in Figure 46. 


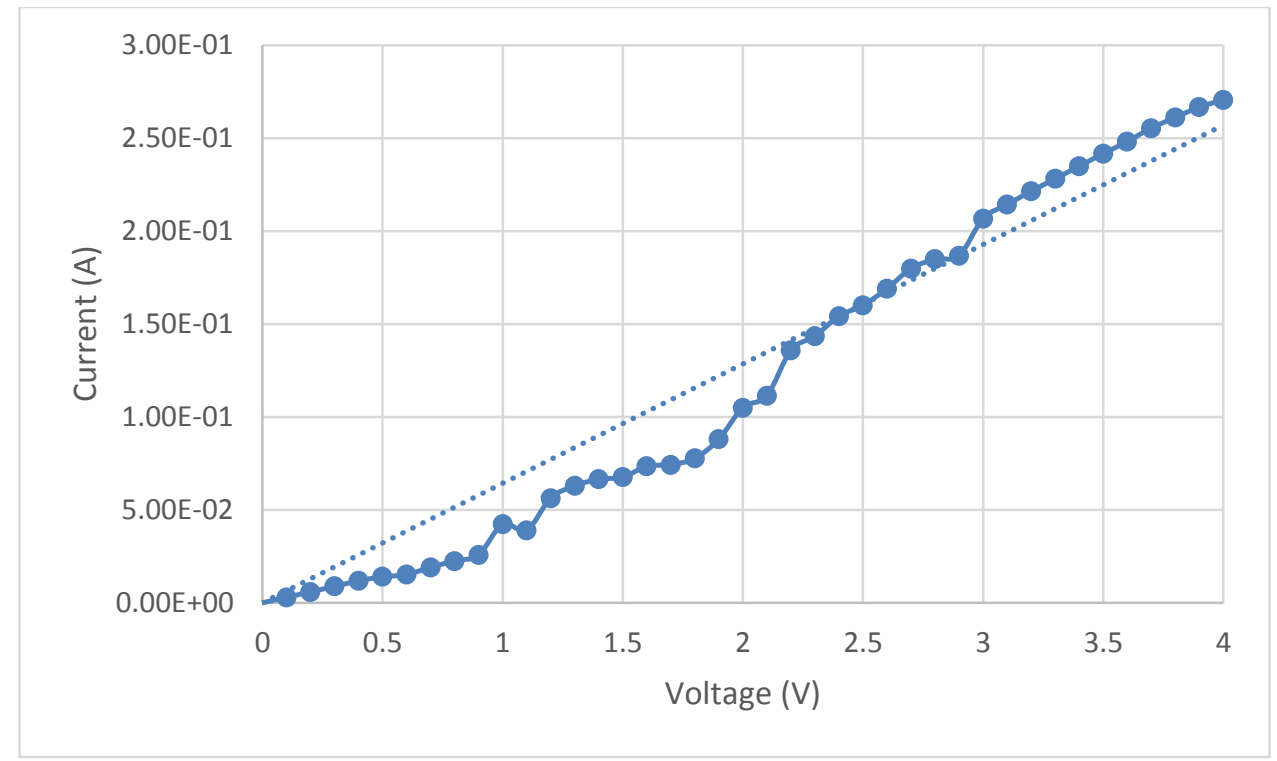

Figure 46: I-V Curve for Aluminum Evaporated onto Glass

\subsubsection{Quantum Dot Layer Deposition using Spin Coating and Devices}

The most common method of layer deposition in the laboratory is spin coating of solutions. This method can be used on small scale and is quick and reproducible. Therefore, it lends itself to device fabrication research in thin films but is limited to small $\left(1 \mathrm{in}^{2}\right)$ substrates. In this study, fabrication using spin coating is used as a stepping stone to inkjet printing. One of the challenges with creating a uniform layer was agglomeration on the substrate but this issue is not characteristic of spin coating alone. One major problem with spincoating is the amount of material that is wasted. On average, more than $95 \%$ of the material is wasted. This procedure will not work for the covalent bonding of the QD to the polymer layer but could be used to deposit polymers doped with hybrid QD/CP particles.[39]

During the early stages of experimentation, many of the devices were plagued with shorting circuitry. The device without the cathode deposited was analyzed using PL 
mapping, and AFM to understand the problem. The PL mapping is displayed in Figure 47 and 48. A photograph of the device under UV irradiation is displayed in Figure 49. The line which travels from the upper-left corner of the mapping to the middle is where the laser was degrading the layers. Figures 47 and 49 show that a uniform layer of the quantum dots was created. The AFM images support that there were not any pin holes in the device but that there may be agglomeration of quantum dots present or even dust particles on the device. To ensure that the level of dust was minimized during the device fabrication, the processing was moved to a small clean hood environment in the Conn Center. The AFM images are shown in Figures 50 and 51.

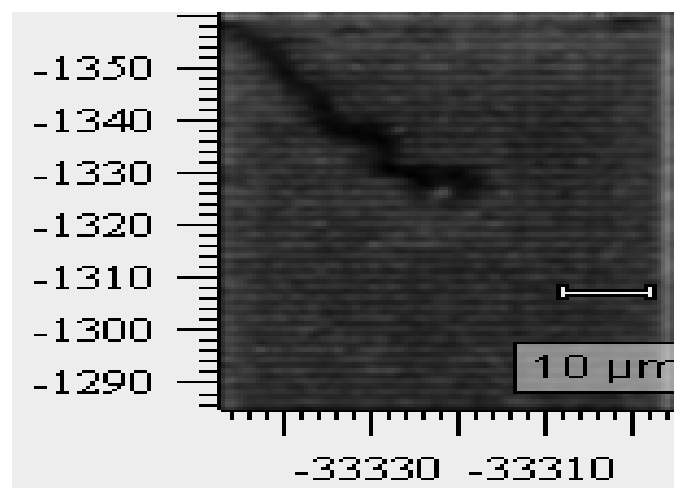

Figure 47: PL Mapping of Spin Coated Device

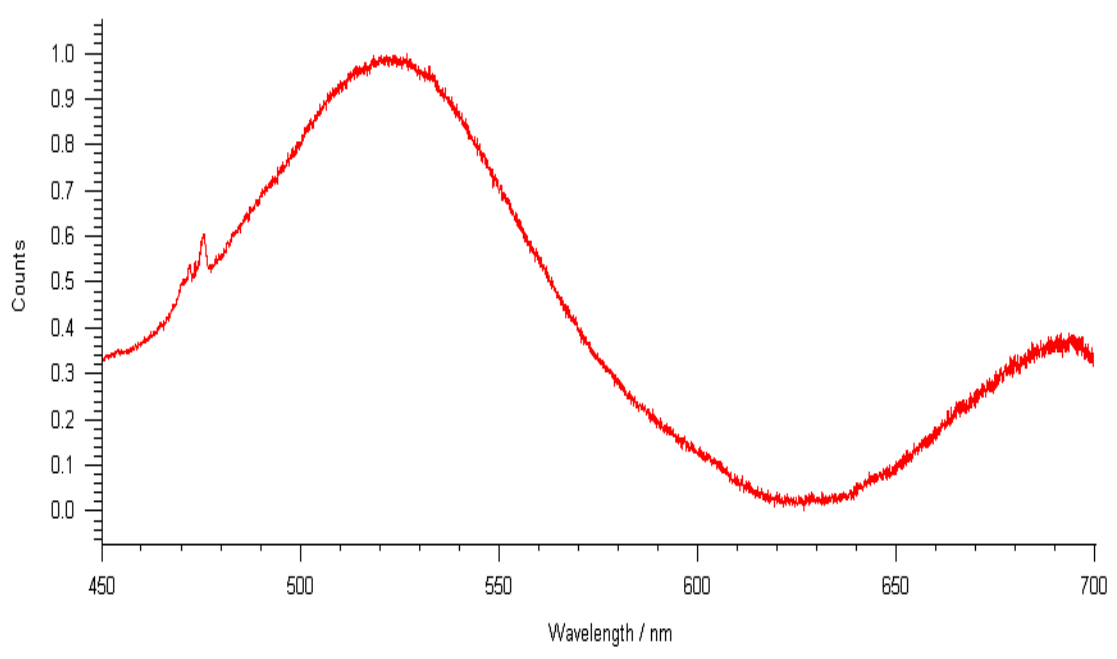

Figure 48: PL Spectroscopy of the Spin Coated Device 


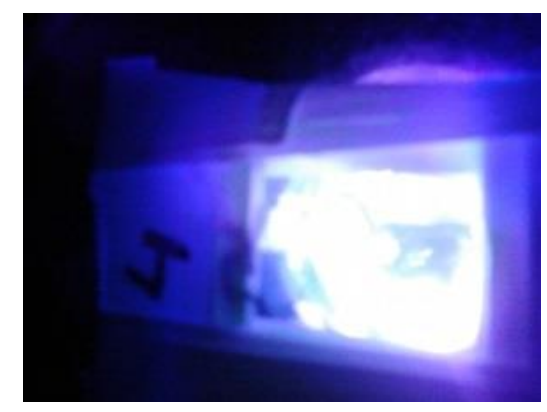

Figure 49: Photograph of Spin Coated Device under UV irradiation

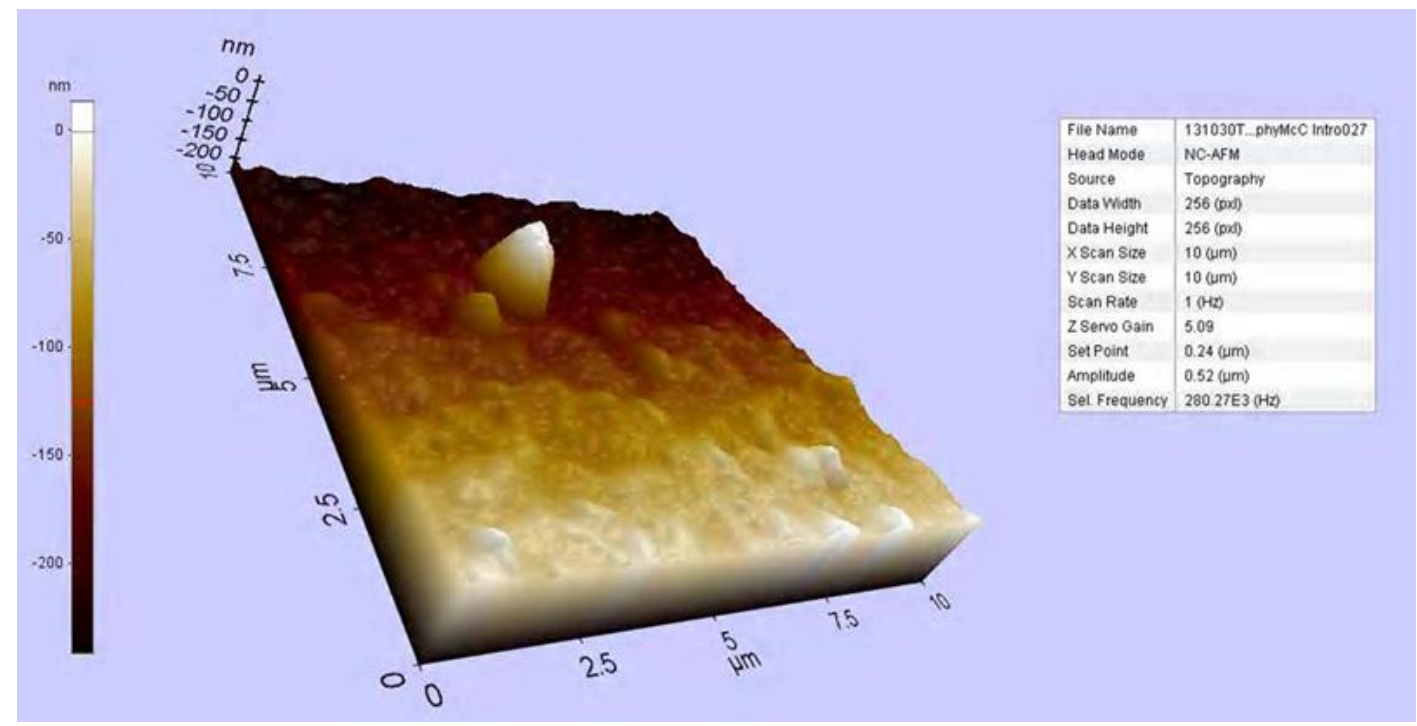

Figure 50: AFM Image of Spin Coated Device 10 $\mu \mathrm{m} \times 10 \mu \mathrm{m}$

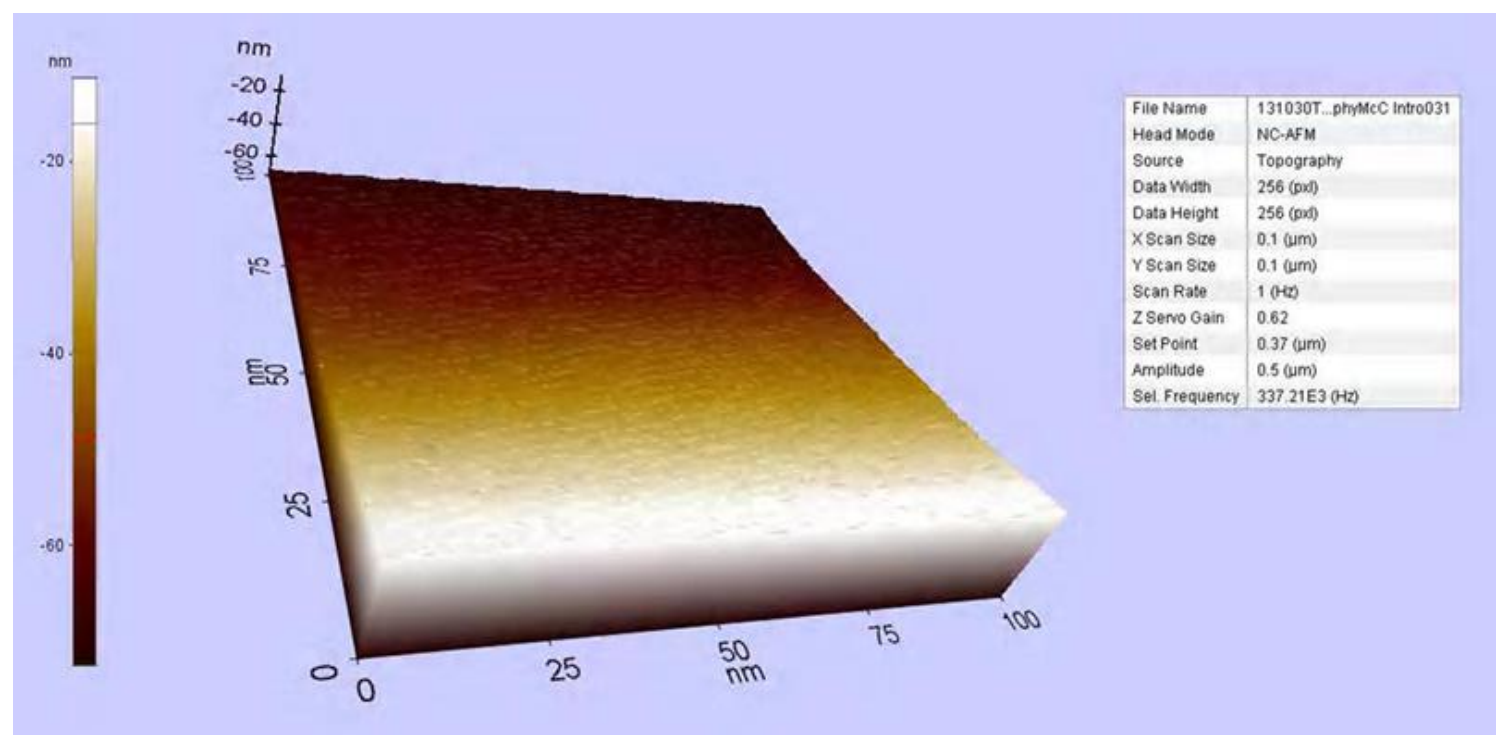

Figure 51: AFM Image of Spin Coated Device 100nm x 100nm 
The I-V Curves of a device are shown in Figure 52 and 53. Figure 52 is the first run of the device. It is clear that the device almost acts as a capacitor as there is no current until $13 \mathrm{~V}$. At this point, the I-V curve takes a constant slope which hints at a shorting of the device. Run 2, Figure 53, reinforces this observation and short circuiting is apparent. Since the PL and AFM analysis techniques concluded that the short circuiting was not a result of discontinuities in the layers, the band gap engineering was not designed well enough to promote a diode behavior. It was also not clear that a continuous layer of the quantum dots was being formed.

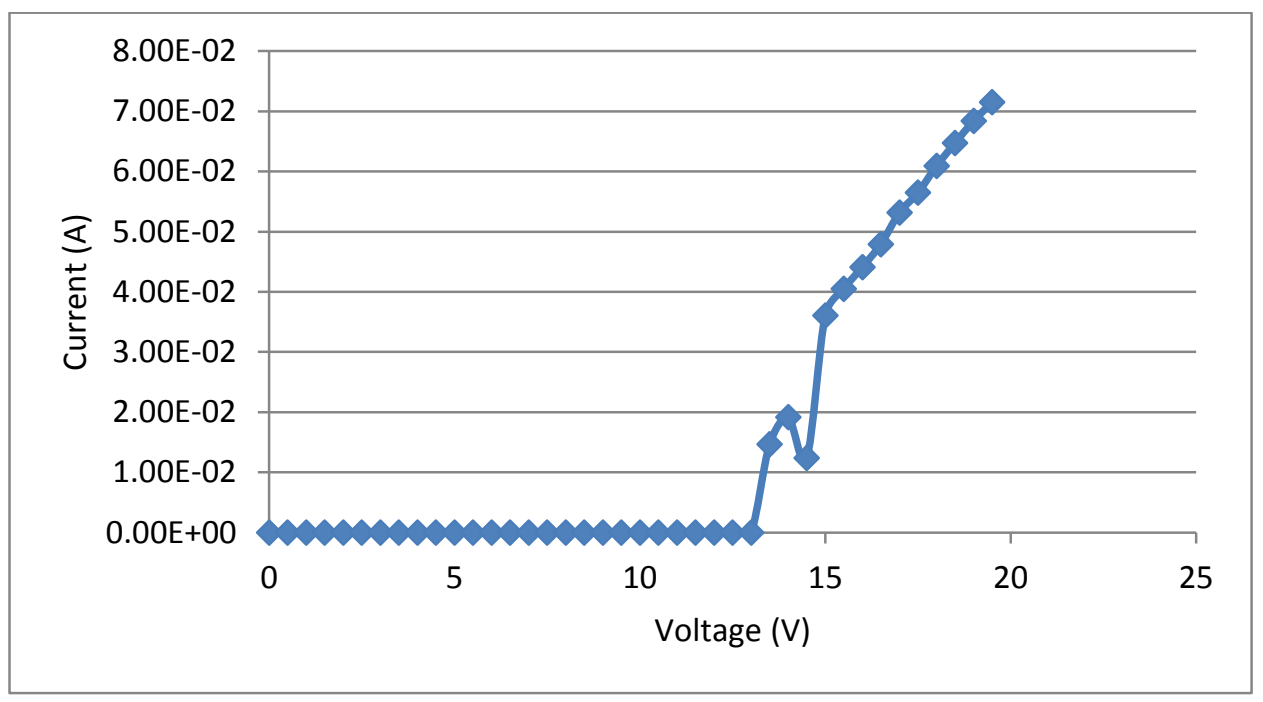

Figure 52: Run 1 of a Spin Coated Original Device Structure 


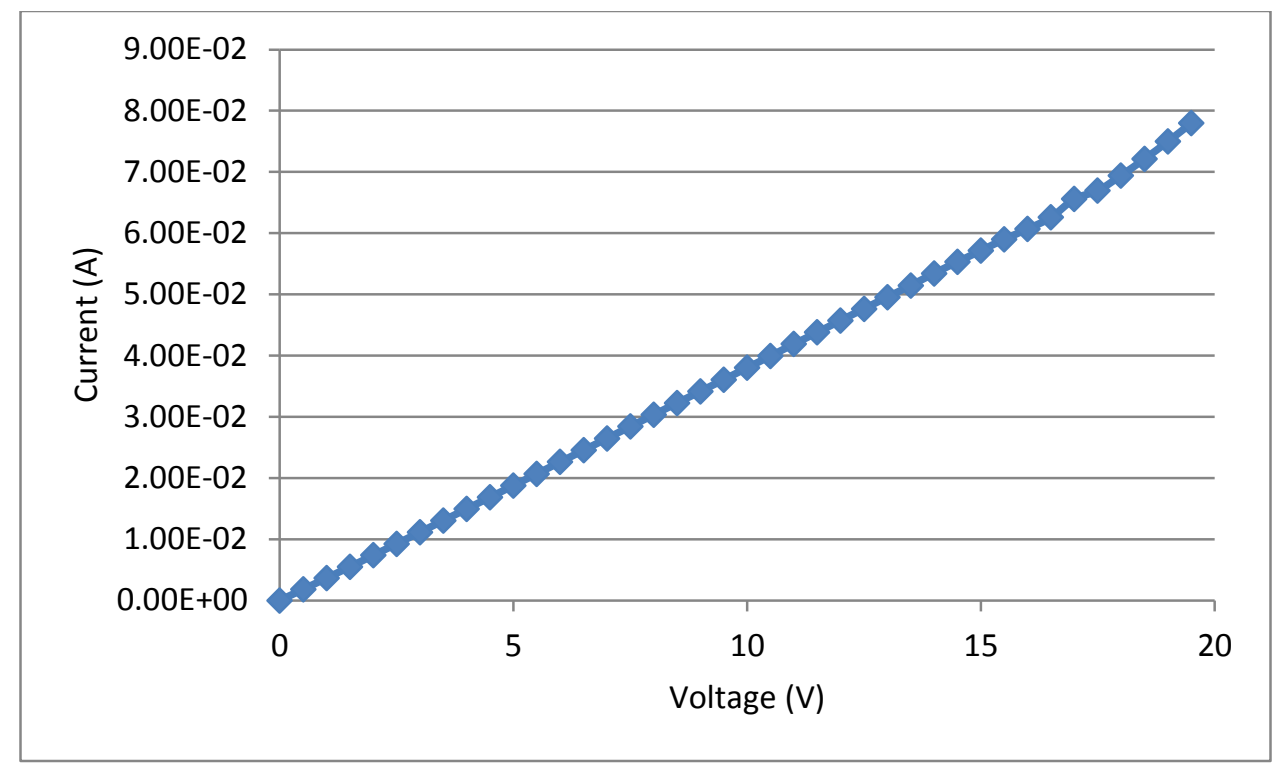

Figure 53: Run 2 of a Spin Coated Original Device Structure

To develop a diode behavior and create a uniform, continuous layer of quantum dots, a second polymer, Poly(9-vinylcarbazole) (PVK), was used in the quantum dot solution and spin coated. PVK was directly added to the QD solution to form a $50 \mathrm{wt} \%$ solution. Optical microscope images of the spin coated PVK/QD layer were taken, a representative image of which is shown in Figure 54. Although the layer did not create a highly uniform layer, the layer was continuous and created devices with diode behavior, displayed in Figures 55 and 56. The purpose of the composite layer was to promote the formation of a monolayer by phase separation of the QDs and PVK. Similarly to the polymer layer deposition, a thermodynamic perspective was developed to better understand the system. 


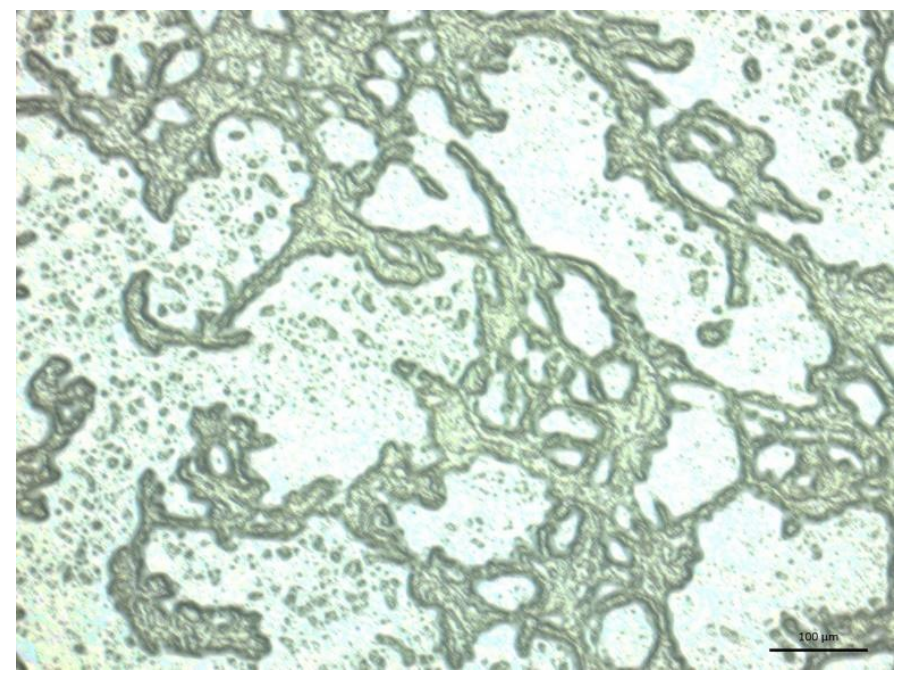

Figure 54: Optical Images of PVK/QD spin coated layer 100 $\mu \mathrm{m}$ Scale Bar

The ligand of the nanoparticle acts as the main means of solubility. Typically, the ligands consist of small carbon chains from 6 to 16 carbons long and are typically soluble in organic solvents such as toluene and chloroform. They can also be functionalized to make them soluble in polar solvents or for the purpose of a reaction. Therefore, the ligand will play a major role in how effective the phase separation will be.

For the polymer, the chain length will play a major role in how it will behave in the solution as the solution becomes more concentrated as discussed in the previous section. Therefore, Equation 6 can help to describe when the critical volume fraction will occur. As shown, it is inversely proportional to the chain length meaning as the chain length increase, the critical volume fraction will decrease. This is important to note because it can be used to control when the polymer will start to create another phase. The critical volume fraction is also dependent on the temperature of the system. As Figure 43 illustrates, an increase in temperature can create an additional phase or get rid of an additional phase based on which critical temperature of the solution is crossed, LCST or UCST. 
The chain length and the temperature could be used to create a separate phase of the polymer. To do this, the chain length will dictate the critical volume fraction of the solution. The temperature of the system can then be altered, positive or negative change depending on the polymer solution, to make the solution unstable and create a different phase. The critical volume fraction can also be reached by evaporation of the solvent as the more conventional way of doing this process.

The nanoparticles on the other hand can be synthesized to tailor the needs of an application. There are some limitations on the different types of ligands that can be used as some do not passivate the surface very well, leading to quick degradation of the quantum dot relative to economical device lifetime or quenching of the quantum dot's photoluminescence and electroluminescence. The most used and reliable ligands are nonfunctionalized, simple chained hydrocarbons. Therefore, the first controllable variable related to the quantum dot's solubility is what ligand surrounds the nanoparticle.

The simplest way of having a direct comparison between the solubilities of the two substances in the solution is the Flory-Huggins interaction parameter because both systems are polymeric in nature. For the process to be successful, the quantum dots need to be in the phase with the solvent. Therefore, the Flory-Higgins interaction parameter for the solvent-quantum dot must be more favorable than the interaction parameter for the quantum dot-polymer but all the interaction parameters must be similar enough to promote solubility in one phase at the temperature and composition during deposition of the solution.

Another aspect to consider when analyzing the thermodynamics of the formation of the monolayer of quantum dots is the energy at the liquid-solid interface once the 
polymer layer has mostly formed. Since the polymer's critical volume fraction is small based on equation (8), the quantum dots will be dispersed in the solvent while it evaporates. Many of the ligands used for the synthesis of quantum dots have very good surfactant qualities that promote the adsorption onto the polymer which can be modeled using the following equation:

$$
c_{i}=c_{b} e^{-\frac{\Delta G_{a d s}^{o}}{R T}}
$$

$\mathrm{c}_{\mathrm{i}}$ is the concentration at the interface; $\mathrm{c}_{\mathrm{b}}$ is the concentration in the bulk solution; $\mathrm{G}$ is the Gibbs free energy of adsorption; $\mathrm{R}$ and $\mathrm{T}$ are the gas constant and the temperature, respectively. The Gibbs free energy is dependent on the electrostatic interaction, covalent bonding, hydrogen bonding, and Van Der Waals interactions.[40] To help promote the construction of the quantum dot layer, the adsorption mechanism should be considered. The concentration of the bulk does not have a large effect considering that all of the solvent will eventually evaporate but where the quantum dots are when the solvent all evaporates is very important to the formation of a monolayer. Therefore, forces such as electrostatic forces can be varied by applying a voltage to promote adsorption by decreasing the Gibbs free energy to make adsorption more favorable. For systems with positive Gibbs free energies of adsorption, decreasing the temperature will favor the adsorption process.

The I-V curves of two different devices with this structure are shown in Figures 55 and 56. With both devices, it appears that the turn-on voltage is between 2 and $3 \mathrm{~V}$. The current of the devices peak in the milliamp range before degrading. Also, these devices did not produce any light which means the electrons were not fully relaxing in the quantum dot layer of the device. Therefore, the design of the structure needed more 
consideration. Since the last attempt approached the problem from the hole transport side of the emissive layer, the electron transport side of the device became of interest.

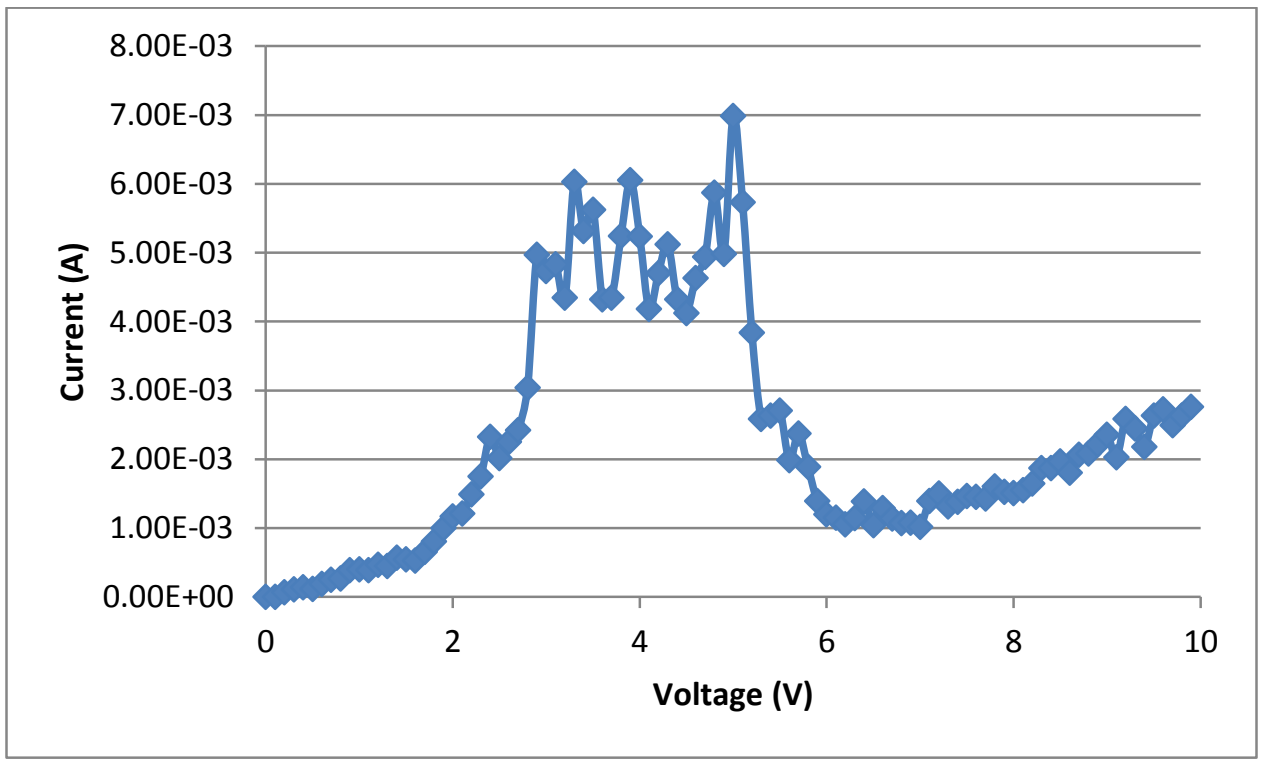

Figure 55: I-V Curve for a PVK/QD Spin Coated Device 1

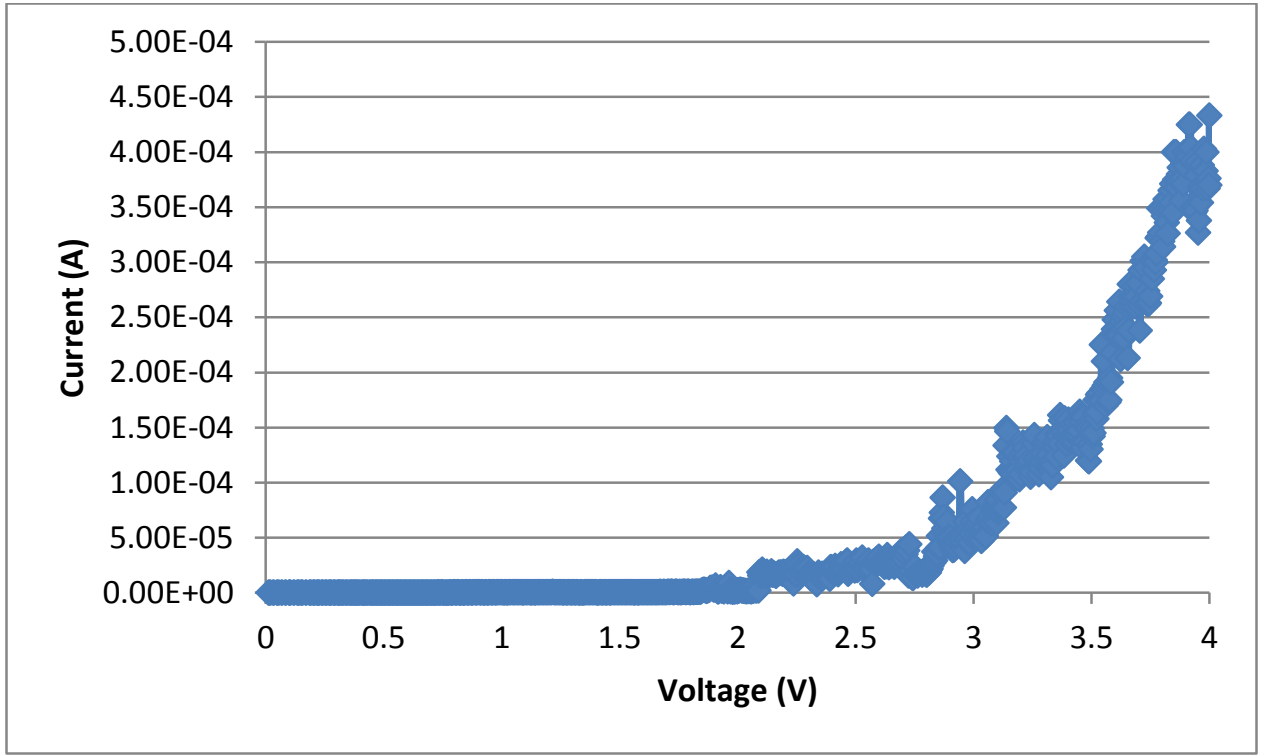

Figure 56: I-V Curve for a PVK/QD Spin Coated Device 2

The use of gold as a cathode is not common but has been used and was very easy to deposit a sufficient layer using the sputter coater. [41] When revisiting the use of gold, it has a very high work function when compared to other typical materials used as 
cathodes which are found in Table 17. The high work function translates to a low ability to provide electron injection into the quantum dot layer because the electrons are tightly bound to the gold atom. The use of aluminum by itself was still not sufficient as its work function is still relatively high. The addition of Alq3 was introduced into the system and did help solve the problem of degradation above $4 \mathrm{~V}$ but still did not produce any light. The new device is displayed in Figure 57. The addition of an electron injection layer was introduced using Lithium Bromide which is also highly reactive in air. Currently, methods are trying to be revised to mitigate the amount of contact with the air while depositing these two layers.

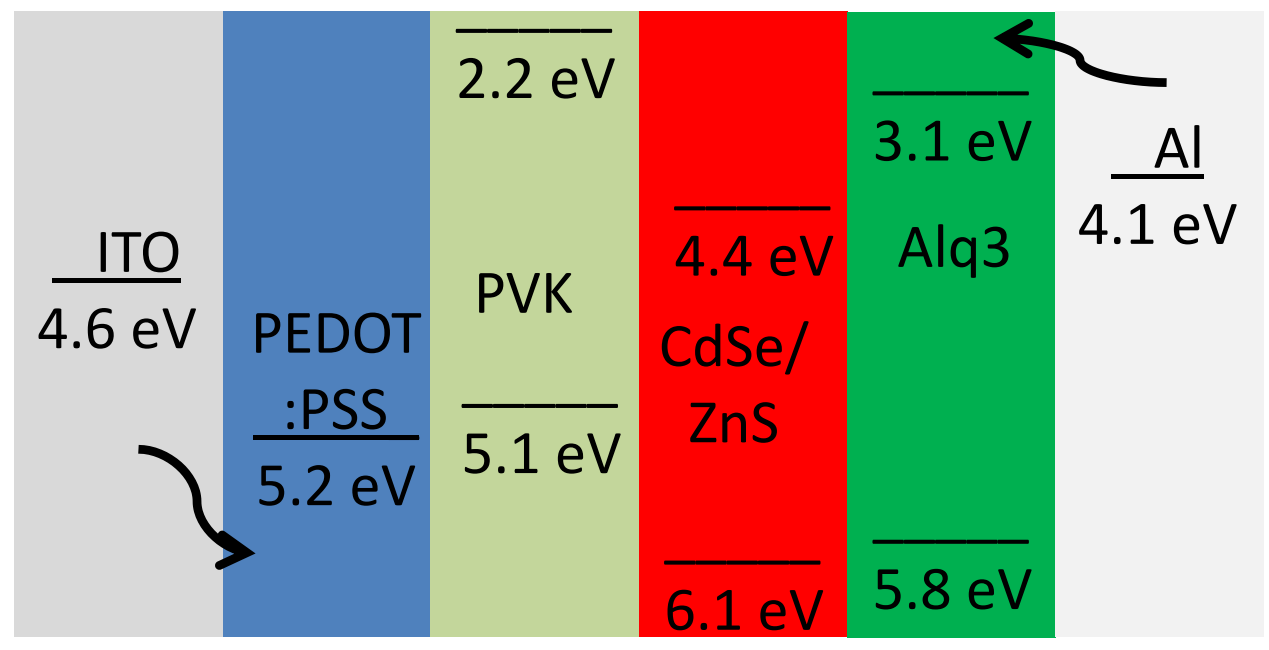

Figure 57: Device with ITO/PEDOT:PSS/PVK/QDs/Alq3/Al

\subsubsection{Quantum Dot Layer Deposition using Inkjet Printing and Devices}

The devices produced using inkjet printing used the quantum dots produced in the previous chapter. The quantum dots produced in house provided the ability to manipulate the solutions for use in the inkjet printer. As can be seen in the photograph in Figure 57, inkjet printing provides a continuous, uniform layer. The quantum dot solutions which 
were used were 1-octadevene based. This was preferential because it developed an easily printable ink when combined with toluene and cyclohexanol. Unfortunately, the ink was extremely difficult to fully dry. Attempts were made using various vacuum ovens at temperatures as high as $180^{\circ} \mathrm{C}$ which probably degraded the PEDOT:PSS polymer and quantum dots. This still did not fully dry the layer and a device could not be formed.

The I-V curve for inkjet printed device with a gold cathode is shown in Figure 58. Not only does is exhibit a short circuited device, it has a high level of noise in the I-V measurements. An attempt was taken to remove the quantum dots from the 1-octadecene but the similarities between the solvent and ligand were too high. The suspension was too stable for centrifugation and washing to be removed fully. Spin Coating was reasoned to be a better initial fabrication method to begin with the developing of the device as there was an initial lack of understanding of producing an operating LED.

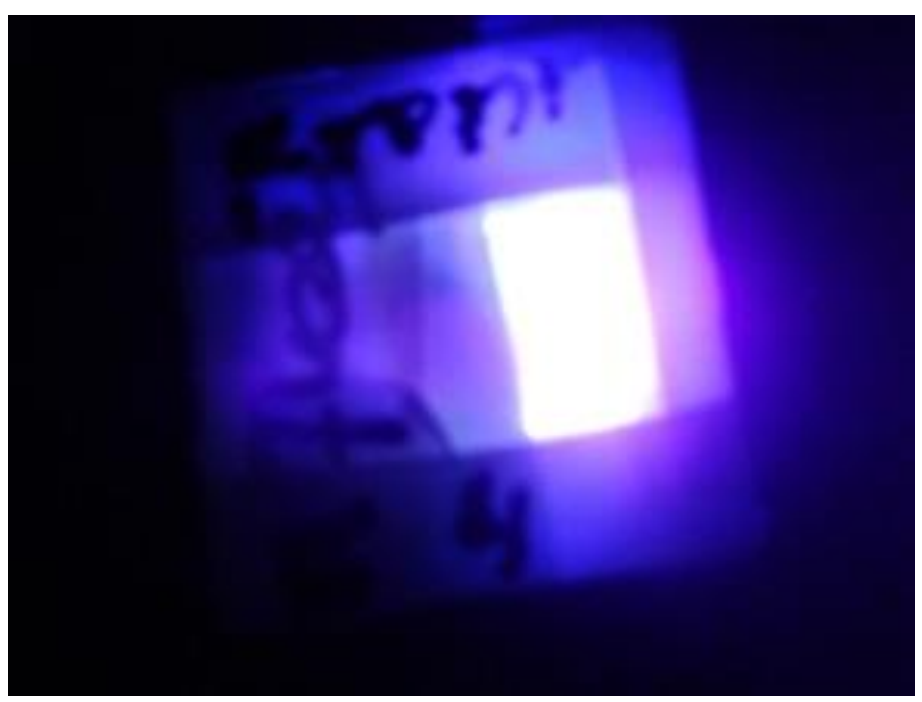

Figure 58: Photograph of an inkjet printed quantum dot layer 


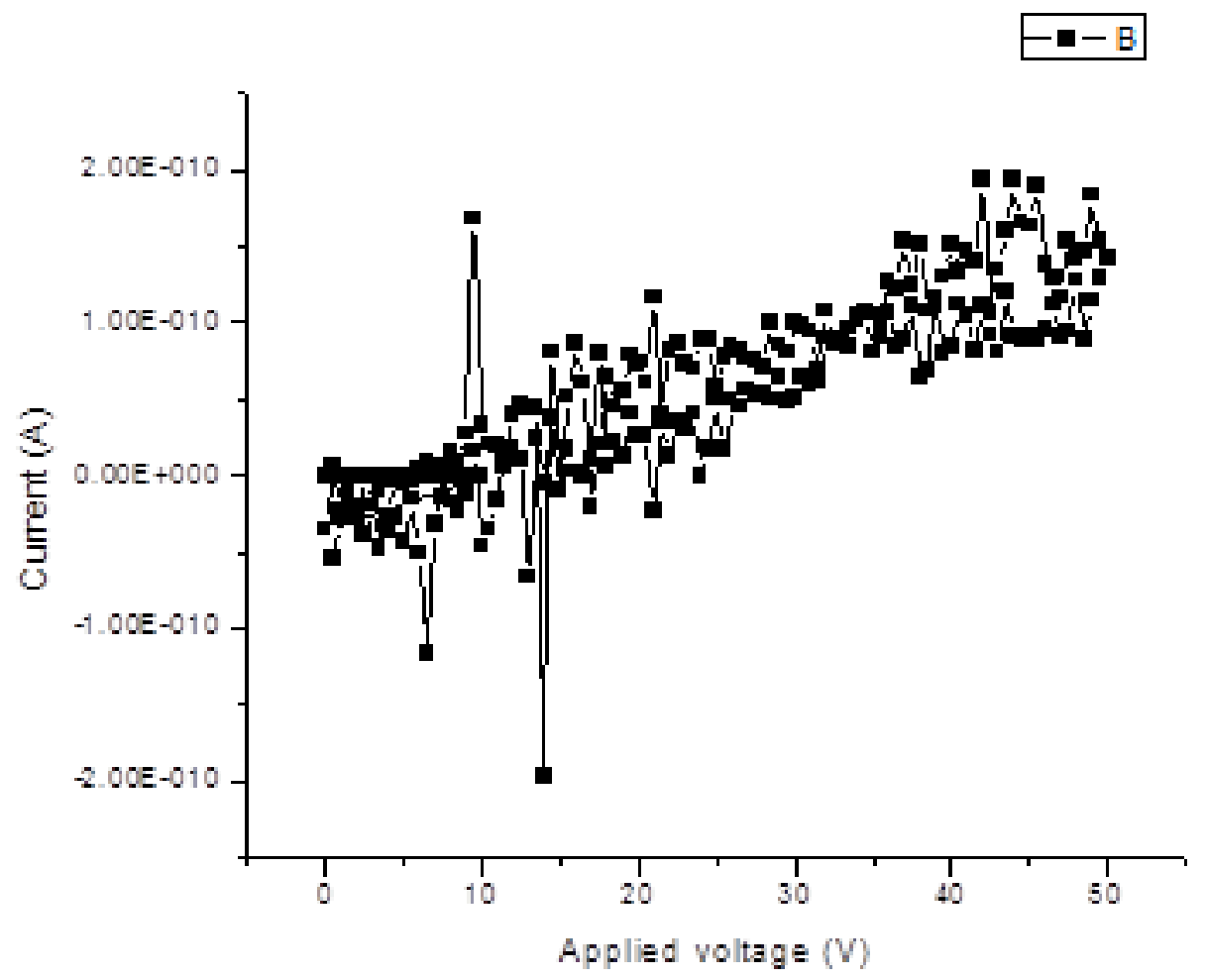

Figure 59: I-V Curve of Inkjet Printed Device

\subsubsection{Quantum Dot Layer Deposition using Drop Casting and Devices}

The use of drop casting was an easy way to test any quantum dot solution in the device. Unfortunately, it did not provide an adequate control of the layer's thickness and uniformity. Often times, the layers were too thick with thickness in the microns and produced "coffee-ring" drying patterns. Although this led to devices that exhibited diode behaviors, the devices were inconsistent and degraded quickly. Two I-V curves of a PVK/QD with a gold cathode are displayed in Figures 59 and 61. The low current was due to the large thickness of the quantum dot layer while the degradation is due to the lack of uniformity in the layer. Although there is an increase in the slope as the voltage 
increases, characteristic to a diode, the second run had no turn on voltage and shows clearly that the device has at least shorted through the quantum dot layer.

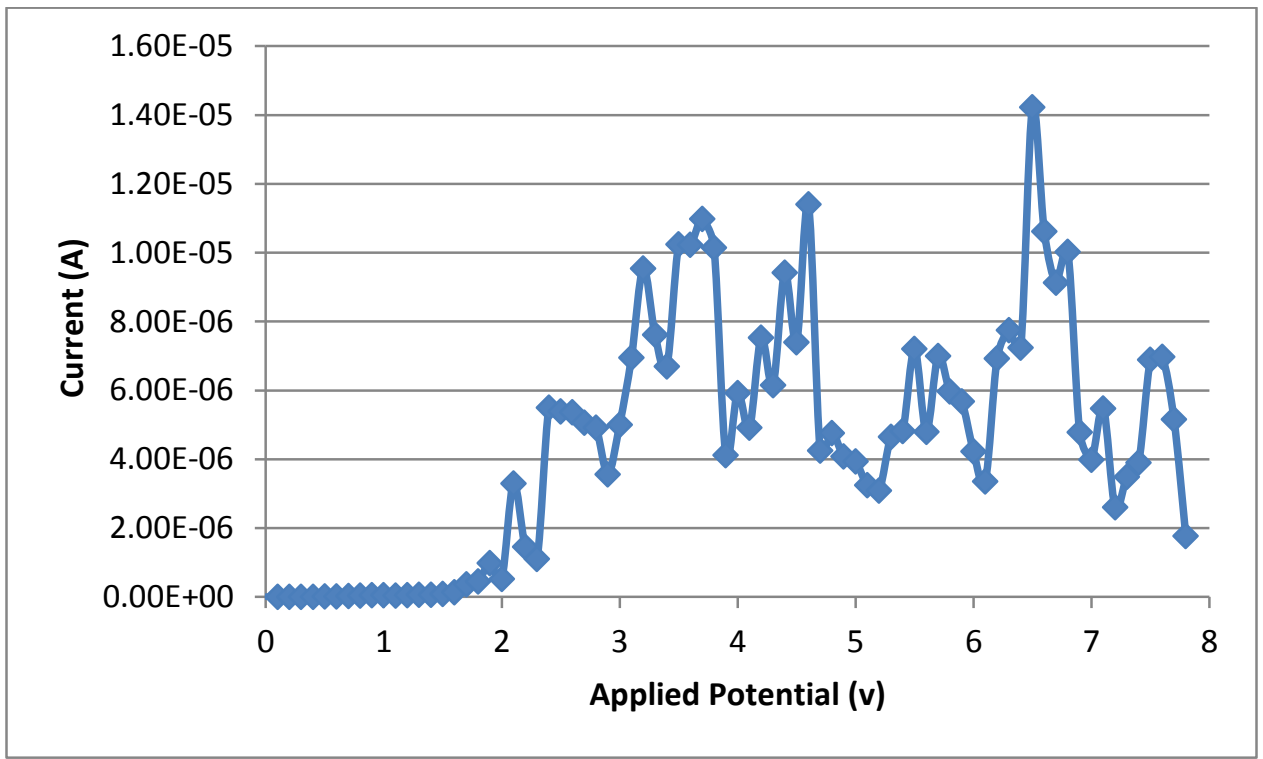

Figure 60: I-V Curve of Drop Casted PVK/QD Device Run 1

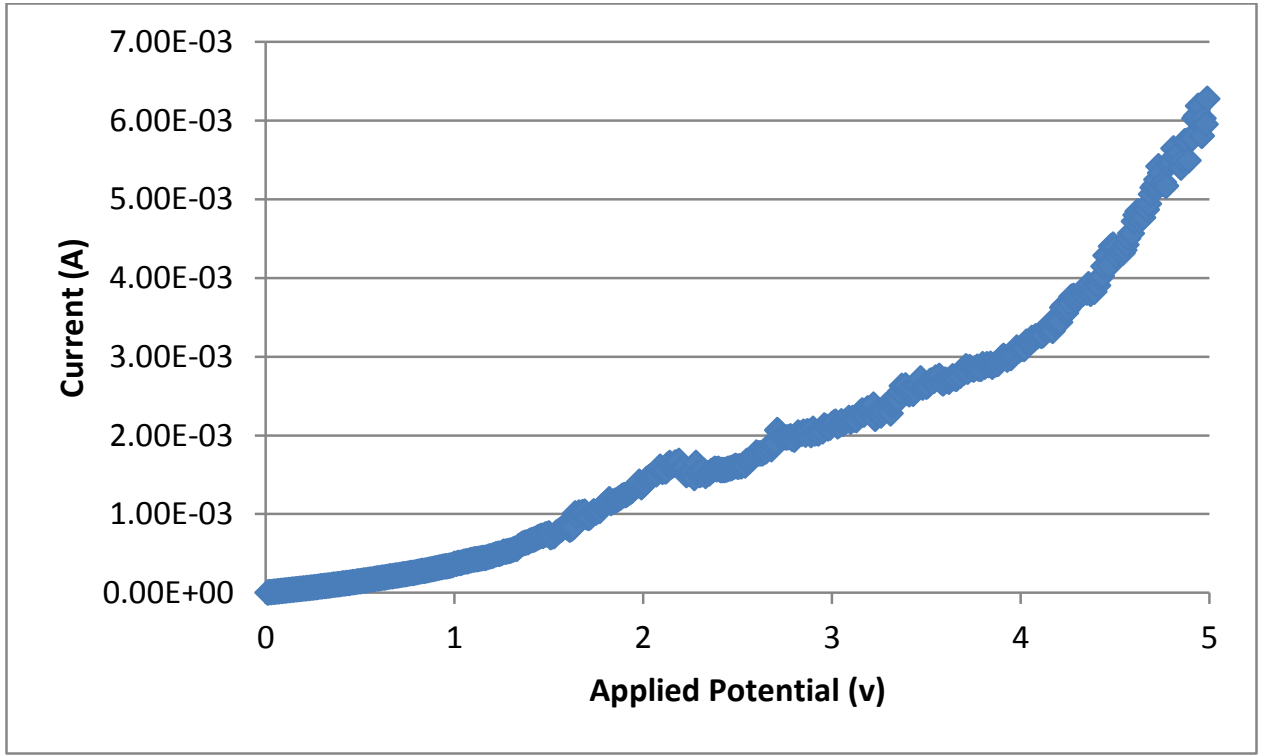

Figure 61: I-V Curve of Drop Casted PVK/QD Device Run 1 


\section{CONCLUSIONS AND FUTURE WORK}

The synthesis of CdSe quantum dots was carried out using a variety of methods including co-precipitation method, reverse micellar method, and hot injection method. Of these three methods, only the hot injection method produced an immediately viable product for application in the hybrid light emitting diode proposed. The co-precipitation method did generate the ability to perform the reverse micellar method which produced $\sim 30 \mathrm{~nm}$ diameter nanorods of mostly amorphous material. These rods were analyzed using XRD, and TEM. The hot injection method using octadecene as the solvent and various ligands, including oleic acid, benzene-1,4-dithiol, mercapto-benzoic acid and TOP, created quantum dots exhibiting photoluminescence in the green region of the visible spectrum. These quantum dots were characterized using UV-Visible Spectroscopy and Photoluminescence Spectroscopy. The oleic acid capped QD had a characteristic wavelength of $560 \mathrm{~nm}$. While the mercapto-benzoic acid and benzene-1,4-dithiol capped QD had a characteristic wavelengths of $510 \mathrm{~nm}$ and $555 \mathrm{~nm}$, respectively, all under the 
same operating conditions. For the purposes of device fabrication, the NN-Labs QDs were mostly used because their performance was much better than quantum dots synthesized in house.

The reverse micellar method has the potential to create high quality quantum dots or rods. One potential method of reaching this goal would be to use a capping ligand to stabilize the micelle instead of an alcohol. This would introduce a material which is readily available to passivate the quantum dot when the reverse micelle system starts to degrade during refluxing. The addition of an inorganic passivation shell would significantly increase the luminescence of the quantum dots produced by the hot injection method, particularly a $\mathrm{ZnS}$ shell. This would allow for a direct comparison to the quantum dots purchased from NN-Labs and a possible replacement of them. A change of the solvent in the hot injection method might allow for a simple solution for inkjet printability and purification of the quantum dot. An increased ability of the purification of the quantum dot from the synthesis solution has the potential to decrease the size distribution of the product.

The fabrication of a hybrid light emitting diode has been developed with the structure ITO/PEDOT:PSS/PVK/CdSe QD/Alq3/Al. The device has exhibited a turn-on voltage from 2-3 $\mathrm{V}$ with currents ranging from 4-5 mA. The PEDOT:PSS layer was deposited using spin coating and analyzed using Photoluminescence Spectroscopy, Atomic Force Microscopy, Optical Microscopy, and Surface Profiling. The layer produce is sufficient for device fabrication. To facilitate proper hole transport and a sufficient quantum dot layer, a PVK/QD composite solution was used to create a monolayer layer 
of quantum dots by phase separation of the solutes in the solution. Alq3 and Al were deposited using thermal evaporation techniques.

The addition of an electron injection layer would help transition the energy of the electron from the aluminum to the Alq3 layer. Lithium fluoride or lithium bromide would be sufficient materials as they have been proven to provide a high level of electron injection with a layer thickness of a few nanometers with the proposed device structure in Figure 62. A better analysis of each layer's thickness would help tune the band gap engineering of the device. This would entail some modification to thermal evaporator as well as switching to contact mode with the AFM to analysis the surface coverage of the quantum dot layer on the PVK. Once a full understanding of the layers is developed, the deposition of the solution layers can be switched to inkjet printing.

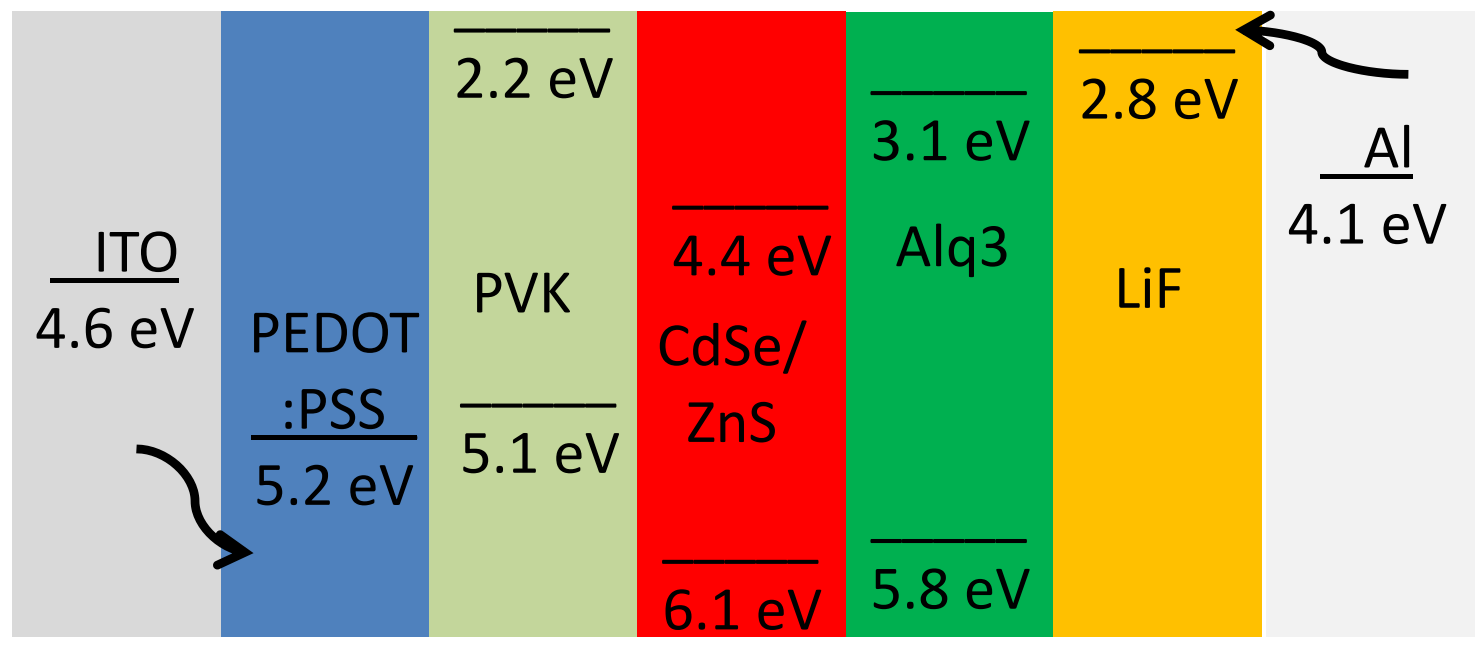

Figure 62: Proposed Device Structure for Future Work 


\section{REFERENCES}

[1] M.T. Nenadovic, T. Rajh, O.I. Micic, The Journal of Physical Chemistry, 89 (1985) 397-399.

[2] J.V. Williams, N.A. Kotov, P.E. Savage, Ind. Eng. Chem. Res., 48 (2009) 4316-4321.

[3] L. Ya-Ju, C. Ching-Hua, K. Chih Chun, L. Po Chun, L. Tien-Chang, K. Hao-Chung, W. Shing-Chung, Selected Topics in Quantum Electronics, IEEE Journal of, 15 (2009) 1137-1143.

[4] D. Bera, L. Qian, T.K. Tseng, P.H. Holloway, Materials, 3 (2010) 2260-2345.

[5] H.J. Round, Electrical World, (1907) 309.

[6] E.F. Schubert, Light-emitting diodes, Cambridge University Press, Cambridge; New York, 2006.

[7] Z.R.M.H. Li, Organic light-emitting materials and devices, CRC/Taylor \& Francis, Boca Raton, 2007. 
[8] J.N. Bardsley, Selected Topics in Quantum Electronics, IEEE Journal of, 10 (2004) 39.

[9] Z.Q. Lin, Chemistry-a European Journal, 14 (2008) 6294-6301.

[10] C.B. Murray, D.J. Norris, M.G. Bawendi, J. Am. Chem. Soc., 115 (1993) 87068715.

[11] B.C. Fitzmorris, J.K. Cooper, J. Edberg, S. Gul, J. Guo, J.Z. Zhang, The Journal of Physical Chemistry C, 116 (2012) 25065-25073.

[12] N. Al-Salim, A.G. Young, R.D. Tilley, A.J. McQuillan, J. Xia, Chem. Mat., 19 (2007) 5185-5193.

[13] Z. Huiguang, P. Arjun, N.B. Denise, J.J. Christopher, L.C. Vicki, Nanotechnology, $21(2010) 255604$.

[14] R.K. Čapek, K. Lambert, D. Dorfs, P.F. Smet, D. Poelman, A. Eychmüller, Z. Hens, Chem. Mat., 21 (2009) 1743-1749.

[15] Y. Shen, M.Y. Gee, R. Tan, P.J. Pellechia, A.B. Greytak, Chem. Mat., 25 (2013) 2838-2848.

[16] O. Samuel Oluwafemi, N. Revaprasadu, New J. Chem., 32 (2008) 1432-1437.

[17] N. Pinna, K. Weiss, H. Sack-Kongehl, W. Vogel, J. Urban, M.P. Pileni, Langmuir, 17 (2001) 7982-7987.

[18] R. Kumar, H. Ding, R. Hu, K.-T. Yong, I. Roy, E.J. Bergey, P.N. Prasad, Chem. Mat., 22 (2010) 2261-2267.

[19] S. Shri Prasad, M. Dinesh Raja, J. Madhavan, Advanced Nanomaterials and Emerging Engineering Technologies (ICANMEET), 2013 International Conference on, 2013, pp. 38-39. 
[20] L. Kang, L. Ae Ri, P. Sang Joon, Microoptics Conference (MOC), 2013 18th, 2013, pp. 1-2.

[21] E. Hao, H. Sun, Z. Zhou, J. Liu, B. Yang, J. Shen, Chem. Mat., 11 (1999) 30963102.

[22] L. Qi, Encyclopedia of Surface and Colloid Science. Second Edition. New York: Taylor \& Francis, (2006) 6183.

[23] D.S. Mathew, R.-S. Juang, Separation and purification technology, 53 (2007) 199215.

[24] N.A. Hamizi, M.R. Johan, Mater. Chem. Phys., 124 (2010) 395-398.

[25] P. Yang, M. Ando, N. Murase, Langmuir, 27 (2011) 9535-9540.

[26] A. Rahmi, A.A. Umar, M.M. Salleh, B.Y. Majlis, M. Yahya, Semiconductor Electronics (ICSE), 2010 IEEE International Conference on, 2010, pp. 229-232.

[27] A.E. Farghal, S. Wageh, A. Abou El-Azm, Journal of Computational Electronics, 10 (2011) 414-423.

[28] P. Wilson, C. Lekakou, J.F. Watts, Organic Electronics, 13 (2012) 409-418.

[29] J. Tardy, M.B. Khalifa, D. Vaufrey, Materials Science and Engineering: C, 26 (2006) 196-201.

[30] H. Yan, P. Lee, N.R. Armstrong, A. Graham, G.A. Evmenenko, P. Dutta, T.J. Marks, J. Am. Chem. Soc., 127 (2005) 3172-3183.

[31] S.W.-K.M.V. Coe, Nature, 420 (2002) 800.

[32] S. Coe-Sullivan, J.S. Steckel, W.K. Woo, M.G. Bawendi, V. Bulović, Advanced Functional Materials, 15 (2005) 1117-1124. 
[33] J.M. Prausnitz, Molecular thermodynamics of fluid-phase equilibria, Prentice-Hall, Englewood Cliffs, N.J., 1969.

[34] K. Kajiwara, W. Burchard, L.A. Kleintjens, R. Koningsveld, Polymer Bulletin, 7 (1982) 191-195.

[35] Y.C. Bae, J.J. Shim, D.S. Soane, J.M. Prausnitz, Journal of Applied Polymer Science, 47 (1993) 1193-1206.

[36] P.J. Flory, The Journal of Chemical Physics, 17 (1949) 223-240.

[37] K. Herman, Gerngross, O. and Abitz, W. Z, Phys. Chem., (1930).

[38] A. Keller, Faraday Discussions of the Royal Society of Chemistry, (1979).

[39] F.C. Krebs, Solar Energy Materials and Solar Cells, 93 (2009) 394-412.

[40] P. Somasundaran, S. Shrotri, L. Huang, Pure and Applied Chemistry, 70 (1998) 621626.

[41] H. Wu, F. Huang, J. Peng, Y. Cao, Organic Electronics, 6 (2005) 118-128. 


\section{VITA}

NAME:

ADDRESS:

DOB:

EDUCATION:

WORK EXPERIENCE:
Michael McCreary

Department of Chemical Engineering University of Louisville

Louisville, KY 40292

Louisville, KY - February 24, 1991

B.S., Chemical Engineering University of Louisville 2009-13

M.Eng., Chemical Engineering University of Louisville 2013-14

Louisville Gas and Electric Research and Development Co-op 2011

Solae, LLC.

Process Engineering Co-op 2011-12

General Electric, Appliances

Business Team Leader Co-op 2013

American Institute of Chemical Engineers American Chemical Society

Tau Beta Pi Honor Society

Golden Key Honor Society 